\title{
WestVirginiaUniversity
}

THE RESEARCH REPOSITORY @ WVU

Graduate Theses, Dissertations, and Problem Reports

2006

\section{Long-term bandwidth estimation}

\author{
Brian E. Masney \\ West Virginia University
}

Follow this and additional works at: https://researchrepository.wvu.edu/etd

\section{Recommended Citation}

Masney, Brian E., "Long-term bandwidth estimation" (2006). Graduate Theses, Dissertations, and Problem Reports. 4247.

https://researchrepository.wvu.edu/etd/4247

This Thesis is protected by copyright and/or related rights. It has been brought to you by the The Research Repository @ WVU with permission from the rights-holder(s). You are free to use this Thesis in any way that is permitted by the copyright and related rights legislation that applies to your use. For other uses you must obtain permission from the rights-holder(s) directly, unless additional rights are indicated by a Creative Commons license in the record and/ or on the work itself. This Thesis has been accepted for inclusion in WVU Graduate Theses, Dissertations, and Problem Reports collection by an authorized administrator of The Research Repository @ WVU. For more information, please contact researchrepository@mail.wvu.edu. 


\title{
Long-Term Bandwidth Estimation
}

\author{
Brian E. Masney \\ Thesis Submitted to the \\ College of Engineering and Mineral Resources \\ at \\ West Virginia University \\ in partial fulfillment of the requirements \\ for the degree of \\ Master of Science \\ in \\ Computer Science \\ Arun A. Ross, Ph.D., Chair \\ Bojan Cukic, Ph.D. \\ Katerina D. Goseva-Popstojanova, Ph.D. \\ Lane Department of Computer Science and Electrical Engineering \\ Morgantown, WV \\ 2006
}

Keywords: bandwidth estimation; self similarity

Copyright (C)2006 Brian Masney 


\title{
ABSTRACT \\ Long-Term Bandwidth Estimation
}

\author{
Brian E. Masney
}

Computer networks are connected with other networks using one or more dedicated circuits. These dedicated circuits have a finite amount of available bandwidth to transport the data. There is a need to predict the future utilization of these circuits so that additional capacity can be added before the circuit becomes saturated. Once the circuit becomes saturated, network packets will be discarded resulting in a poor end-user experience. It typically takes several weeks or, in some cases, several months for an order of additional capacity to be installed. A network planner should, therefore, know in advance when additional capacity will be required. The goal of this thesis is to develop a system that can predict the traffic utilization of a circuit six months into the future. If this goal can be accomplished, then network planners will have the ability to optimize the provisioning cycle of network capacity. The thesis describes a method to build a model for estimating network traffic data in the future based on current network characteristics. It also proposes a technique for anomaly detection that can be used to determine if the model has to be updated as traffic characteristics may change over time. The performance of the model has been evaluated on six different real-world datasets. Experimental results indicate the strengths and limitations of the proposed model. 


\section{ACKNOWLEDGEMENTS}

I would like to thank Dr. Arun Ross for all of the help that he has given me while I was a graduate student at WVU. The classes that I have taken from him were wonderful and he has given me great guidance while I worked on this thesis. I would also like to thank all of the other wonderful faculty and staff at WVU that helped me while I was a graduate student.

I would like to thank my employer, Ntelos, for allowing me to go to graduate school while I worked full time. The experience of going to school full time and working full time helped me to manage my time more efficiently.

I would also like to thank my family and friends for being there for me. Their love and support is what helped to make my experience at WVU a very pleasant and rewarding experience. 


\section{CONTENTS}

I. Introduction and Literature Review . . . . . . . . . . . . . . 1

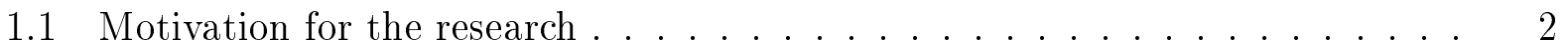

1.2 Contributions of this thesis $\ldots \ldots \ldots \ldots \ldots$

1.3 Properties of network traffic $\ldots \ldots \ldots \ldots \ldots \ldots$

1.3.1 Fractals . . . . . . . . . . . . . . . . . . 4

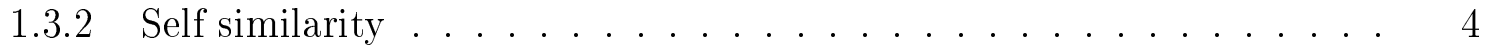

1.3.3 The random walk . . . . . . . . . . . . . . . 5

1.3.4 Brownian motion . . . . . . . . . . . . . . . 6

1.3 .5 Sierpinski triangle . . . . . . . . . . . . . . 8

1.3 .6 The Hurst parameter . . . . . . . . . . . . . . . . . . . 10

1.3.7 Self similarity in Ethernet traffic . . . . . . . . . . . . . 11

1.3.8 The Hurst parameter and queue utilization . . . . . . . . . . . 13

1.3.9 The auto-correlation and auto-covariance functions $\ldots \ldots \ldots \ldots$

1.3.10 Long-Range dependence . . . . . . . . . . . . . . . . . 15

1.3 .11 How the data changes over time $\ldots \ldots \ldots \ldots \ldots$

1.4 Time series introduction $\ldots \ldots \ldots \ldots \ldots \ldots$ 
1.4 .1 Decomposition using STL . . . . . . . . . . . . . 18

1.4 .2 Stationary time series . . . . . . . . . . . . . . . . . . . 19

1.4 .3 The lag operator $\ldots \ldots \ldots \ldots \ldots \ldots$

1.4 .4 Seasonality . . . . . . . . . . . . . . . . . . . . 22

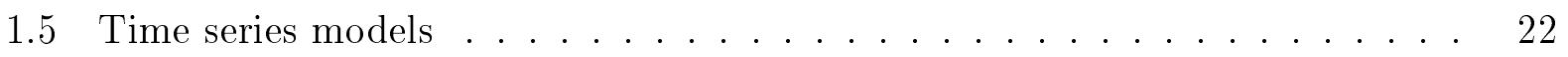

1.5.1 Autoregressive $(\mathrm{AR}) \ldots \ldots \ldots \ldots \ldots \ldots \ldots$

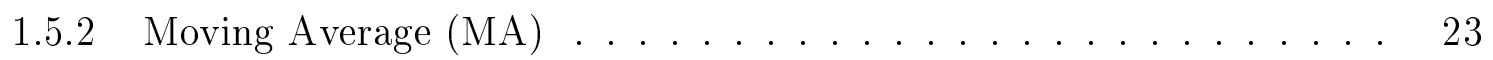

1.5.3 Autoregressive Moving Average (ARMA) . . . . . . . . . . . 24

1.5.4 Autoregressive Integrated Moving Average (ARIMA) . . . . . . . 25

1.5.5 Fractionally Integrated ARMA (FARIMA) $\ldots \ldots \ldots \ldots \ldots$

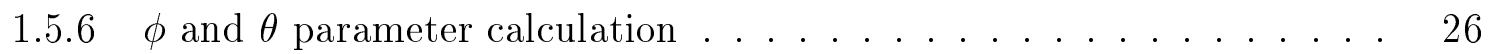

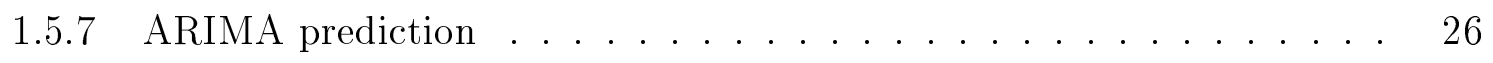

1.6 Short-Term bandwidth estimation . . . . . . . . . . . . . . . 27

1.7 Long-Term bandwidth estimation $\ldots \ldots \ldots \ldots \ldots \ldots$

1.7.1 Long-Term forecasting using wavelets . . . . . . . . . . 27

1.8 Principal Component Analysis (PCA) . . . . . . . . . . . . . 30

1.9 Independent Component Analysis $(\mathrm{ICA}) \ldots \ldots \ldots \ldots$

II. Introduction to the Data Sets . . . . . . . . . . . . . . . . 35

2.1 Data set information . . . . . . . . . . . . . . . 35

$2.1 .1 \quad$ Data polling . . . . . . . . . . . . . . . . 35

2.1 .2 Data set statistics . . . . . . . . . . . . . . 36 
2.2 Introduction to RRDTool $\ldots \ldots \ldots \ldots \ldots$

2.3 RRD database parameters $\ldots \ldots \ldots \ldots \ldots \ldots$

2.3.1 Data Source $(\mathrm{DS})$ parameters . . . . . . . . . . . . . . 38

2.3.2 Round-Robin Archive (RRA) parameters . . . . . . . . . . . . 39

2.3 .3 Time period calculation $\ldots \ldots \ldots \ldots$. . . . . . . . . 40

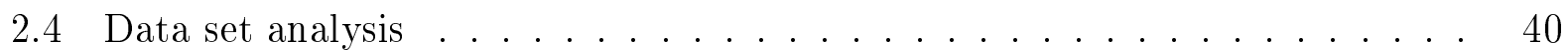

2.4 .1 Auto-correlation function . . . . . . . . . . . . . . . . . 40

2.4 .2 Self-Similarity test . . . . . . . . . . . . . . . . . . 42

2.4 .3 Test for seasonality $\ldots \ldots \ldots \ldots \ldots$. . . . . . . . . . . . . 42

2.4 .4 Test for long-range dependence $\ldots \ldots \ldots \ldots \ldots$

2.4.5 Signal decomposition using STL . . . . . . . . . . . . . 45

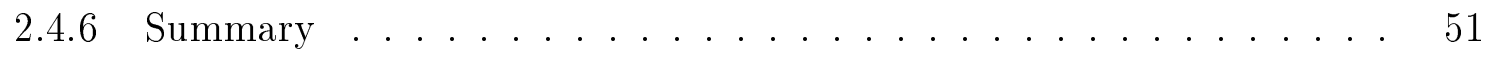

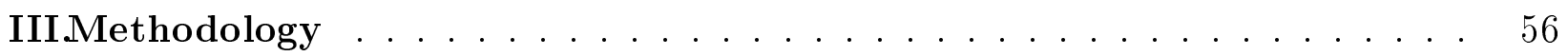

3.1 Smoothing using Principle Component Analysis (PCA) . . . . . . . . . 57

3.2 Smoothing using Independent Component Analysis (ICA) $\ldots \ldots \ldots$

3.3 Anomaly detection $\ldots \ldots \ldots \ldots \ldots \ldots$

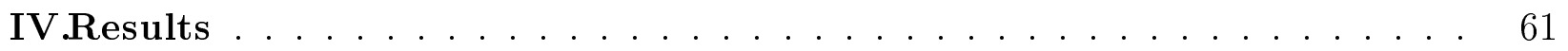

$4.1 \quad$ Prediction error . . . . . . . . . . . . . . . . . . . 61

4.2 Traffic prediction using ARIMA . . . . . . . . . . . . . . 62

4.3 Traffic prediction using PCA and ARIMA . . . . . . . . . 63

4.4 Traffic prediction using ICA and ARIMA . . . . . . . . . . 69 
$4.5 \mathrm{PCA} / \mathrm{ICA}$ vs the STL trend component . . . . . . . . . . . 72

4.6 Anomaly detection results ....................... 75

4.7 Summary . . . . . . . . . . . . . . . . . 77

4.8 Future Work . . . . . . . . . . . . . . . . 77 


\section{LIST OF FIGURES}

1.1 A simple example of two routers that are linked together using one dedicated circuit. A router is typically used to connect two or more networks. The dedicated circuit that connects the two routers could be a physical cable or it could be a wireless transport. The utilization of several circuits is what was examined in this thesis. . . . . . . . . . . . . . .

1.2 This diagram shows an example of three ideal fractals. The first column shows the initiator and the second column shows the generator. The initiator is the initial state of the model. The generator is ran recursively on the model. The third column shows the state of the model after three iterations. Notice that the smaller sections of the model are the same shape as the larger sections. From $[7] \ldots \ldots \ldots \ldots \ldots \ldots \ldots \ldots \ldots \ldots \ldots \ldots$

1.3 IP traffic viewed under different time scales [24]. Notice that the traffic is bursty at all 5 time scales. Averaging the data set over a period of time will not remove the spikes in the graph. From $[24] \ldots \ldots \ldots \ldots$

1.4 This figure shows 5 iterations of the 1D random walk. Each random walk contains 1,000 samples. Knowledge of the previous values will not help you predict the future values. . . . . . . . . . . . . 
1.5 This figure shows 5,000 iterations of the 2D Brownian Motion. Knowledge of the previous values will not help you predict the future values. . . . . . .

1.6 This figure shows the process that is used to generate the Sierpinski triangle. A triangle is drawn and each of the three points is labeled 1, 2 and 3. Then a random point $\left.\left(x_{0}, y_{0}\right)\right)$ is selected as the initial point. A random number in the set $(1,2,3)$ is drawn. The value of $\left(x_{i}, y_{i}\right)$ is the point that is halfway between $\left(x_{i-1}, y_{i-1}\right)$ and the Nth point in the outer triangle. Note: The points are not connected by lines in the algorithm. The lines were drawn to illustrate

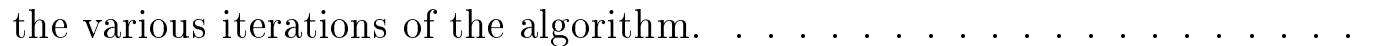

1.7 It is possible to use a random process to generate the Sierpinski triangle, which is a self-similar object. . . . . . . . . . . . . . . .

1.8 The packet arrival times for various source/destination pairs [24]. The top diagram shows the aggregated traffic for source 10 . The remaining diagrams show the traffic between source 10 and some of its destinations. Each source/destination pair exhibit the Noah Effect [8], which is data that has high variability. Aggregating these source/destination pairs onto a single circuit produce the Joseph Effect [8], which is data that has a high long-range dependence (LRD) or self similarity. From $[24] \ldots \ldots \ldots$ 
1.9 Queue utilization with different values of the Hurst parameter $(H)$ [20], [21]. As the level of self similarity increases, the burstiness of the traffic increases as well. The increased traffic bursts have a direct impact on the queue utilization. From $[21] \ldots \ldots \ldots \ldots \ldots \ldots$

1.10 The auto-covariance function of two different processes that exhibit Short Range Dependence (SRD) and Long-Range Dependence (LRD). A SRD process will decay exponentially while a LRD process will decay hyperbolically. From $[34] \ldots \ldots \ldots \ldots \ldots \ldots$

1.11 This figure shows the various values of $\alpha$. Notice that as the value of $\alpha$ increases, the graph will reach it's asymptote more quickly. The graph of a heavy-tailed distribution will be hyperbolic in nature. . . . . . . . . . . 16

1.12 This figure shows an example of the output of the STL algorithm. This algorithm can be used to split the time series into three components: i) the trend component, ii) the seasonal component and iii) the remaining component. 19

1.13 This figure shows an example of a time series where one iteration of the lag - 1 difference operator was performed. This operator subtracts the previous value in the time series from the current value. . . . . . . . . . . . . . 21

1.14 The traffic prediction error using Long-Term Forecasting with wavelets. From

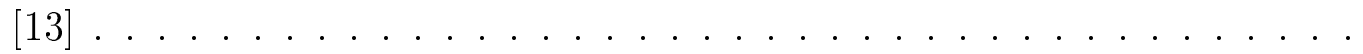

1.15 The Fast Fourier Transform (FFT) of three network traces. There are strong periods at the 12 and 24 -hour time scale. From $[13] \ldots \ldots \ldots$ 
1.16 The terms that are used in the expansion of $c_{3}(5)$ in the a-trous wavelet transformation [13]. The coarser wavelet resolutions will require the values from a wider range of time which will help to produce a smoother signal. From

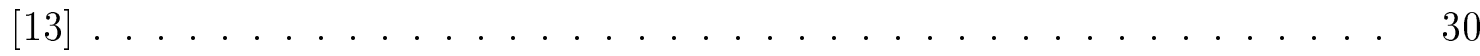

1.17 The six-month prediction of the traffic. From $[13] \ldots \ldots$

1.18 Principal Component Analysis (PCA) will rotate the coordinate system so that the variance is maximized across each dimension. This figure shows an example of how the coordinate system may be rotated using PCA. . . . . .

1.19 Independent Component Analysis (ICA) transform the data set so that the yth order statistics are optimized. This figure shows an example of how the data set may be transformed using ICA. . . . . . . . . . . .

1.20 ICA assumes that there are two or more independent source signals, $\mathbf{x}(t)$, that are mixed linearly in some unknown fashion (A) to form one or more mixed signals, $\mathbf{s}(t)$. To determine the independent components $(\mathbf{y}(t))$, the inverse mixing matrix (W) needs to be calculated that gives the components that are the most independent of each other. From $[6] \ldots \ldots \ldots \ldots$

2.1 The graphs of the six data sets that were analyzed in this paper. . . . . . 37

2.2 The auto-correlation function of the six data sets, time in days. . . . . . . . 41

2.3 The inbound traffic by the day of the week. There appears to be a seasonal component on the weekends to these data sets. A significance test was performed to verify this hypothesis. . . . . . . . . . . . . . . 43 
2.4 The outbound traffic by the day of the week. There appears to be a seasonal component on the weekends to these data sets. A significance test was performed to verify this hypothesis. . . . . . . . . . . . . 44

2.5 Hill's estimator on the inbound traffic. . . . . . . . . . . . 46

2.6 Hill's estimator on the outbound traffic. . . . . . . . . . . . . . 47

2.7 There are times when the Hill plot will not stabilize. In cases like this, the Hill plot will need to be computed multiple times until it stabilizes. These two graphs show the computed Hill Plot for the inbound traffic on data set \#4. 48

2.8 The classical decomposition of the inbound traffic in the six data sets using the STL algorithm [4]. Recall that the classical decomposition of a time series can be represented as the sum of three components: i) the trend component, ii) the seasonal component and iii) the random noise component. . . . . . .

2.9 The classical decomposition of the outbound traffic in the six data sets using the STL algorithm [4]. Recall that the classical decomposition of a time series can be represented as the sum of three components: i) the trend component, ii) the seasonal component and iii) the random noise component. . . . . . . 50

3.1 A example of some anomalies that may be present in the traffic utilization. . 60

4.1 An ARIMA model of the data sets was generated. This model was used to predict the traffic six months into the future. The results are shown for data sets $\# 0-2 . \ldots \ldots \ldots \ldots \ldots \ldots \ldots$ 
4.2 An ARIMA model of the data sets was generated. This model was used to predict the traffic six months into the future. The results are shown for data

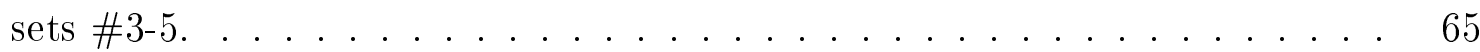

4.3 The inbound PCA Components for the six data sets. . . . . . . . . . 66

4.4 The outbound PCA Components for the six data sets. . . . . . . . . . 66

4.5 The eigenvectors whose eigenvalues represent the top $95 \%$ of the total variance were kept. The data was filtered using the remaining eigenvectors. These are the generated graphs for the inbound data on the six data sets. . . . . .

4.6 The eigenvectors whose eigenvalues represent the top $95 \%$ of the total variance were kept. The data was filtered using the remaining eigenvectors. These are the generated graphs for the outbound data on the six data sets. . . . . . . 68

4.7 The data was smoothed using Principal Component Analysis (PCA). An ARIMA model of the smoothed data was generated for each data sets. This model was used to predict the traffic six months into the future. The results are shown for data sets $\# 0-2 . \ldots \ldots \ldots . \ldots \ldots$

4.8 The data was smoothed using Principal Component Analysis (PCA). An ARIMA model of the smoothed data was generated for each data sets. This model was used to predict the traffic six months into the future. The results are shown for data sets \#3-5. ................... 71 
4.9 The data was smoothed using Independent Component Analysis (ICA). An ARIMA model of the smoothed data was generated for each data sets. This model was used to predict the traffic six months into the future. The results are shown for data sets $\# 0-2 . \ldots \ldots \ldots$. . . . . . . . . 73

4.10 The data was smoothed using Independent Component Analysis (ICA). An ARIMA model of the smoothed data was generated for each data sets. This model was used to predict the traffic six months into the future. The results are shown for data sets \#3-5. . . . . . . . . . . . . . 74

4.11 The PCA filtered data was compared against the STL trend component. The STL trend component is much smoother in appearance. The auto-correlation function of both data sets was computed. This shows that a seasonal component still exists in the PCA filtered data set. Similar results were obtained for the ICA filtered data set. . . . . . . . . . . . . . . . 76

4.12 The original data set had an overall daily prediction error of $26 \%$. If the model is rebuilt after the first two weeks when the correlation drops below 0 , then the overall daily prediction error is $13 \%$. Rebuilding the model a second time drops the overall daily prediction error to $8 \% . \ldots . . . . . . . .76$ 


\section{LIST OF TABLES}

1.1 The various types of circuits that may be offered by an Internet Service Provider $($ ISP) $\ldots \ldots \ldots \ldots \ldots \ldots \ldots \ldots \ldots \ldots \ldots$

2.1 The numeric representation of the six data sets that were analyzed in this paper. 36

2.2 The Data Source (DS) parameters of the RRD database. . . . . . . . . . 38

2.3 The Round-Robin Archive (RRA) parameters of the RRD database. . . . . . 39

2.4 The Hurst parameters for the six data sets. Three methods were used to calculate the Hurst parameter: i) the rescaled range statistic (R/S) method, ii) the variance time plot method and iii) the index of dispersion for counts . 42

2.5 The numeric representation of the six data sets by the day of the week. . . 52

2.6 The p-values from the t-tests that were performed on data sets \#0-2 to determine the significance of the mean and variance between each day of the week. This data shows that there is a seasonal component on the weekends to the majority of these data sets. The outbound traffic for data set \#2 is the only data set that does not have a seasonal component. . . . . . . . . . 53 
2.7 The p-values from the t-tests that were performed on data sets \#3-5 to determine the significance of the mean and variance between each day of the week. The analysis show there is a weekend seasonal component within all

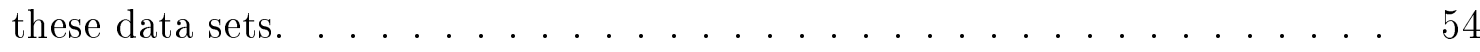

2.8 The estimated $\alpha$ parameter for the six data sets. Recall that $\alpha$ values less than 1 represent data sets that exhibit long-range dependence. . . . . . . 55

4.1 The estimated ARIMA parameters for the six data sets. The root Mean Square Error (MSE) along with the corresponding prediction error are also

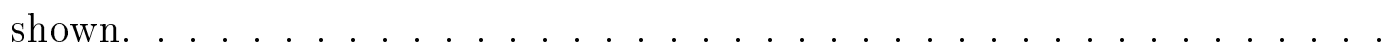

4.2 The standard deviation and the proportion of variance for the 7 principal components in the inbound data sets. . . . . . . . . . . .

4.3 The standard deviation and the proportion of variance for the 7 principal components in the outbound data sets. . . . . . . . . . . .

4.4 The estimated ARIMA parameters for the six data sets that were smoothed using Principal Component Analysis (PCA). . . . . . . . . .

4.5 The estimated ARIMA parameters for the six data sets that were smoothed using Independent Component Analysis (ICA). . . . . . . . . . . . 72

4.6 The correlations between the PCA and ICA smoothed data was compared against the STL trend component. It appears that the seasonal component

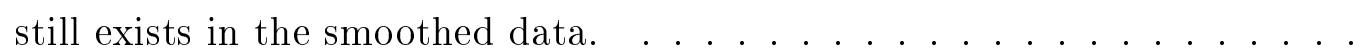


4.7 A summary of the weekly percentage error for the three ARIMA prediction

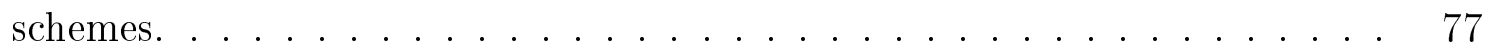




\section{INTRODUCTION AND LITERATURE REVIEW}

An Internet Service Provider (ISP) is a business or organization that offers access to the Internet. Today's ISPs offer various forms of Internet access that vary from dialup to highspeed lines such as T3s (or higher). Table 1.1 shows the types of circuits that ISPs typically

offer to their customers. Wikipedia [32] gives a more thorough listing of the various types of circuits that may be offered by an ISP.

\begin{tabular}{|c|c|}
\hline Circuit Type & Maximum Speed \\
\hline Dialup & up to $56 \mathrm{kbps}$ \\
ISDN & $64 \mathrm{kbps} / 128 \mathrm{kbps}$ \\
ADSL & up to $8 \mathrm{Mbps}$ \\
Frame Relay & $8 \mathrm{kbps}-45 \mathrm{Mbps}$ \\
DS1/T1 & $1.5 \mathrm{Mbps}$ \\
DS3/T3 & $45 \mathrm{Mbps}$ \\
OC3 & $155 \mathrm{Mbps}$ \\
\hline
\end{tabular}

Table 1.1: The various types of circuits that may be offered by an Internet Service Provider (ISP).

The Internet does not have a central authority that is the backbone. Instead, there are several Tier-1 ISPs [44] around the world that represent the core backbone of the Internet. Each Tier-1 ISP will peer with other Tier-1 ISPs in regions where their service areas overlap (usually for free). The larger regional ISPs will usually peer with one or more Tier-1 ISPs that are in their coverage area. This relationship allows smaller ISPs to peer with regional ISPs for Internet access. 
Figure 1.1 shows an example of a dedicated circuit. A dedicated circuit will make a connection between two or more networks. Each network will have at least one router at the edge of the network that is used to communicate with the other networks' routers.

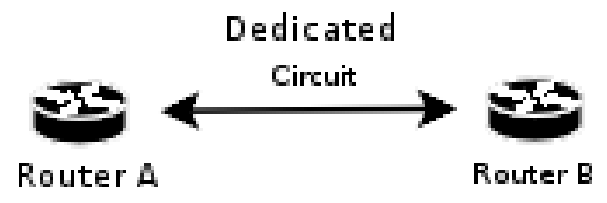

Figure 1.1: A simple example of two routers that are linked together using one dedicated circuit. A router is typically used to connect two or more networks. The dedicated circuit that connects the two routers could be a physical cable or it could be a wireless transport. The utilization of several circuits is what was examined in this thesis.

\subsection{Motivation for the research}

Each one of these peering relationships require one or more dedicated circuits with a finite amount of bandwidth. If the circuits do not have enough available bandwidth for the current demand, then the routers will silently discard packets [38]. This will result in a poor end-user experience. On the other hand, if the links have too much available bandwidth, then the network operator is paying extra money for bandwidth they are not using. An ideal solution is somewhere in between these two cases.

When a network operator decides they need to upgrade or order a new circuit, an order is placed for additional network capacity. Depending on the region and the type of circuit, it may take several weeks to several months for the order to be completed. This is due to the fact that it may require new facilities to be added to the central office or it may require fiber optic cable to be installed across a particular region. For example: The time to 
complete an order may take an extended period of time if new fiber optic cable needs to be installed across the bottom of an ocean. Due to this long provisioning cycle, it is important for network planners to know in advance when they will need to order additional network capacity.

Most network planners currently use their intuition and previous experiences to determine when they need to place the order. They will sometimes base the decision on when the utilization of the circuit reaches a particular threshold. The threshold may be arbitrarily

chosen or it may be based on Service Level Agreements (SLAs) with their customers [11]. Ideally it would be nice to have an automated system that monitors the network and inform the network planner when the capacity of a particular circuit is expected to reach a certain threshold.

\subsection{Contributions of this thesis}

The goal of this thesis is to develop a system that can predict the traffic utilization of a circuit six months into the future. If this goal can be accomplished, then network planners will have the ability to optimize the provisioning cycle of network capacity. In addition, this work can also have benefits beyond ordering new circuits: it can be used to determine when a maintenance window should be planned some time in the future.

This thesis provides a novel technique to build a model of the data. This model is used to predict the future values in the time series. It also proposes a technique for anomaly detection that can be used to determine when the model should be regenerated as the characteristics 
of the traffic may change over time.

\subsection{Properties of network traffic}

\subsubsection{Fractals}

Fractals are unique structures that were first described by Mandelbrot [9]. Euclidean geometry was not suitable to easily model the structures that occurred in nature. Mandelbrot starts out his book [9] by stating "Clouds are not spheres, mountains are not cones, coastlines are not circles, and bark is not smooth, nor does lightning travel in a straight line." There needed to be a way to capture the underlying components of these structures.

The concept of fractals started in the late 19th century whenever mathematicians were generating fractals. Figure 1.2 shows an example of three ideal fractals: a von-Koch curve, a Sierpinski triangle and a Menger sponge [7]. A fractal starts out with an initiator. There exists a preprogrammed generator that is run recursively on the structure. The generator will produce very complex structures after only a few iterations.

\subsubsection{Self similarity}

Self similarity occurs whenever the same pattern occurs over a period of time under different time scales. This is the definition of a fractal that was described in the previous section.

Leland et al. found that packet arrival times of network traffic exhibits the self similarity property [8]. The data in a self-similar process is highly correlated, thus averaging the data set over a period of time will not remove the self similarity. Figure 1.3 shows the traffic trace 


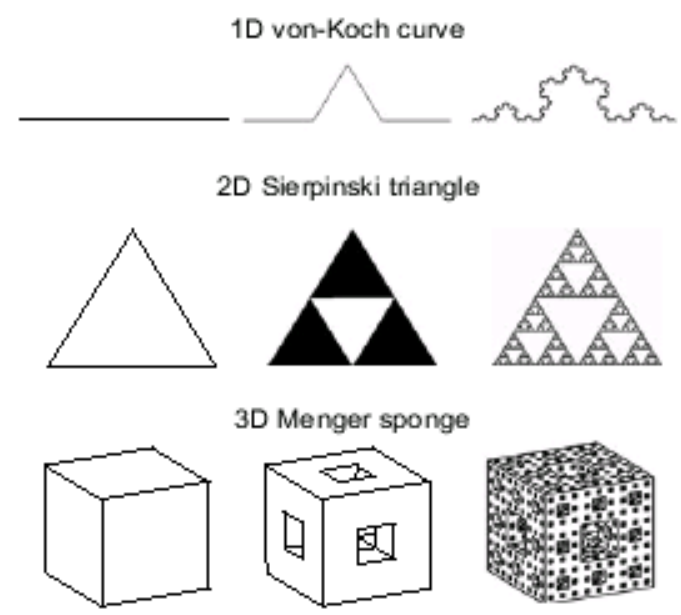

Figure 1.2: This diagram shows an example of three ideal fractals. The first column shows the initiator and the second column shows the generator. The initiator is the initial state of the model. The generator is ran recursively on the model. The third column shows the state of the model after three iterations. Notice that the smaller sections of the model are the same shape as the larger sections. From [7]

of a specific network interface viewed under various time scales. Notice how all of the time scales show spikes in the data.

\subsubsection{The random walk}

The random walk is the first random process that will be examined. The process has a starting point, which is denoted $x_{0}$. There are two choices for the value of $x_{1}$ : it can either move to the left or the right. This process is continued successively until the desired number of iterations has been reached. Knowledge of the previous values will not help you predict the future values. Figure 1.4 shows an example of 5 random walks.

The random walk can be characterized by the following equation:

$$
x_{i}=x_{i-1}+\lambda
$$



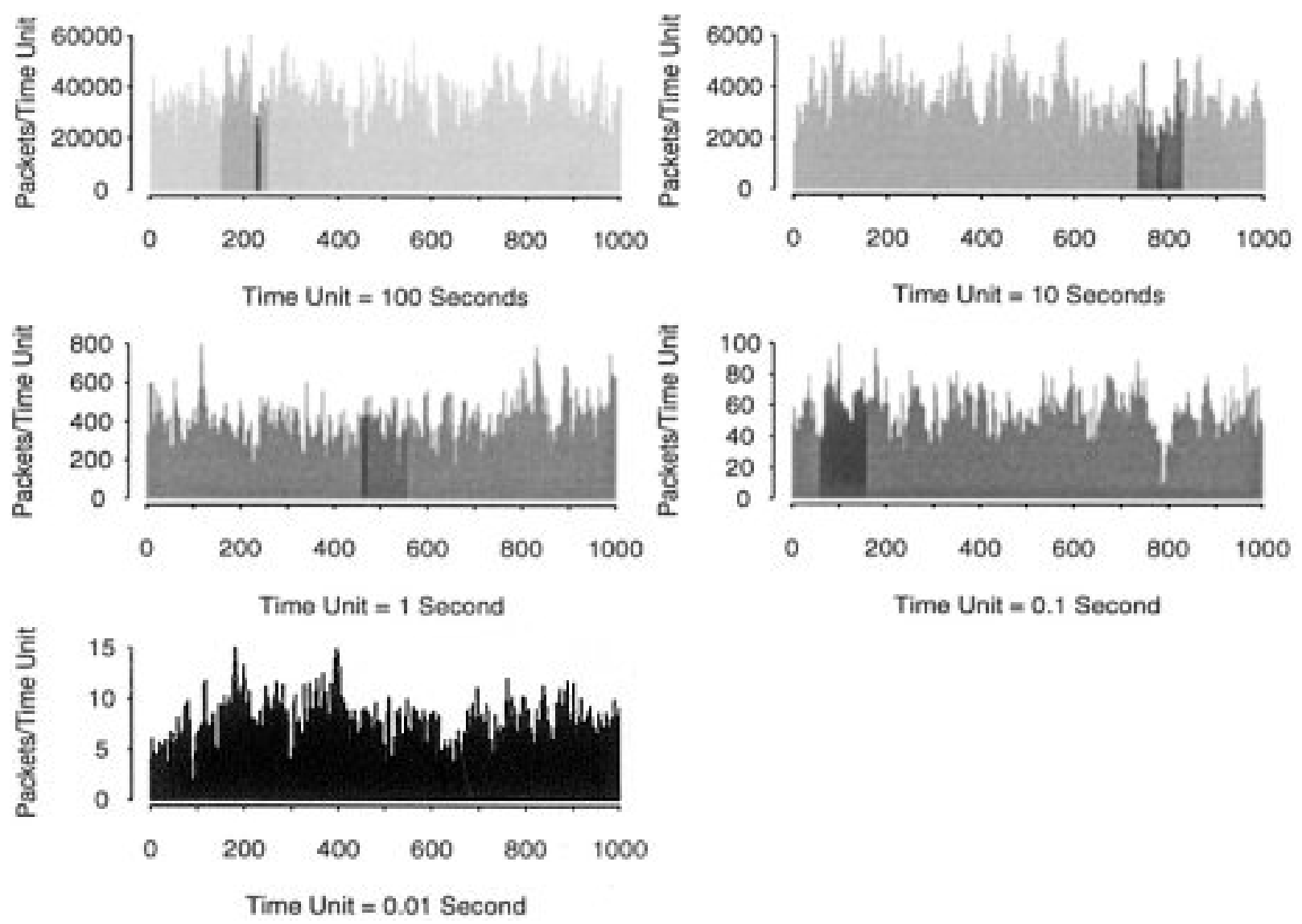

Figure 1.3: IP traffic viewed under different time scales [24]. Notice that the traffic is bursty at all 5 time scales. Averaging the data set over a period of time will not remove the spikes in the graph. From [24]

where $\lambda$ can have the values -1 or 1 and each value has an equal probability of 0.5 .

\subsubsection{Brownian motion}

Brownian motion is the next random process that will be examined. This motion was found to be naturally occurring by Robert Brown in the early 1800 s. When viewing an object under a microscope, it was noted that the molecules appear to move in a random fashion. The molecules that move will bump into nearby molecules, which will cause them to move as well. 


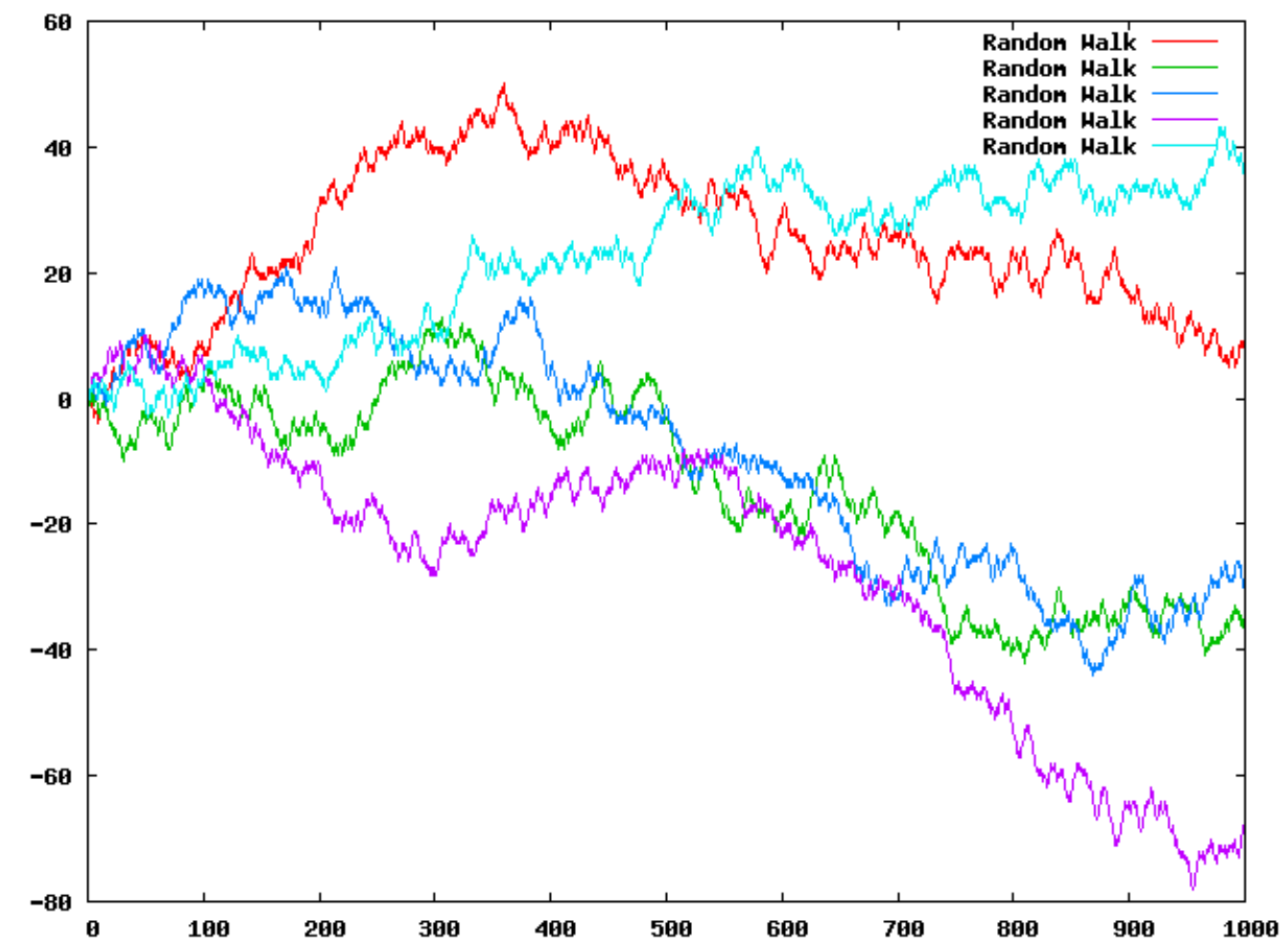

Figure 1.4: This figure shows 5 iterations of the 1D random walk. Each random walk contains 1,000 samples. Knowledge of the previous values will not help you predict the future values. 
Figure 1.5 shows an example of Brownian motion. The process has a starting point, which is denoted $x_{0}$. There are eight choices for the value of $x_{1}$ : it can move up, down, left, right and across all four diagonals. Knowledge of the previous values will not help you predict the future values.

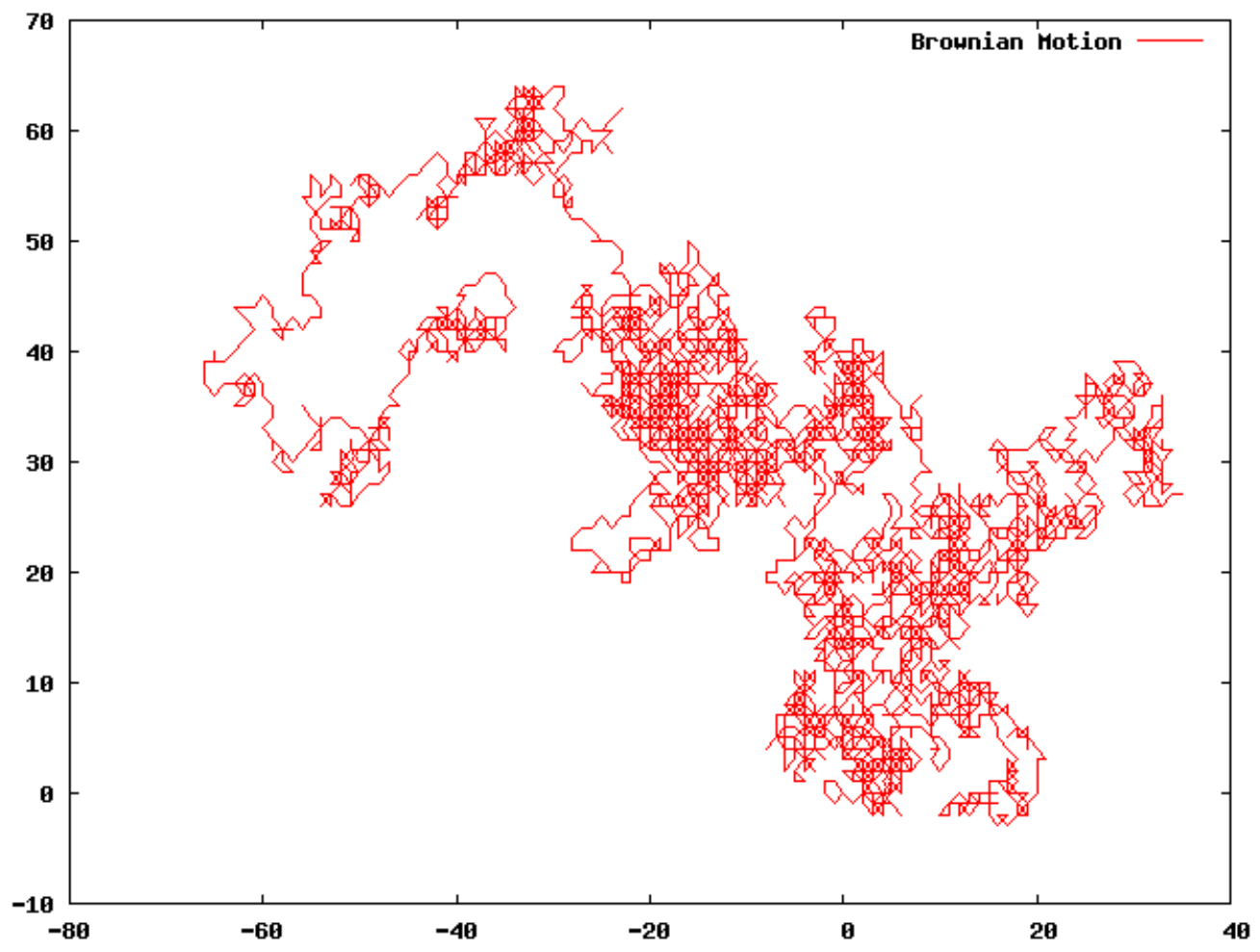

Figure 1.5: This figure shows 5,000 iterations of the 2D Brownian Motion. Knowledge of the previous values will not help you predict the future values.

\subsubsection{Sierpinski triangle}

Barnsley [3], [15] found that it is possible to have a random process generate a self-similar image. One example is generating the Sierpinski triangle. Recall that a self-similar process has two parts: the initiator and the generator.

Figure 1.6 demonstrates the algorithm that is used. The initiator starts out with a 
triangle anywhere in space. Label the three points of the triangle 1, 2 and 3 respectively. These represent the outer section of the Sierpinski triangle. Then mark a starting point $\left(x_{0}, y_{0}\right)$ anywhere at random on the plane. The point can be inside or outside of the outer triangle.

The generation of $\left(x_{i}, y_{i}\right)$ is characterized by the following equation:

$$
\left(x_{i}, y_{i}\right)=\frac{\left(x_{i-1}, y_{i-1}\right)+\lambda[N]}{2}
$$

where $\mathrm{N}$ is a random variable that is in the set $(1,2,3)$, where each value has equal prior probabilities. $\lambda$ is the three points in the outer triangle and $\lambda[N]$ is the Nth point in that triangle. Figure 1.7 shows an example where 100, 1,000 and 10,000 iterations were performed.

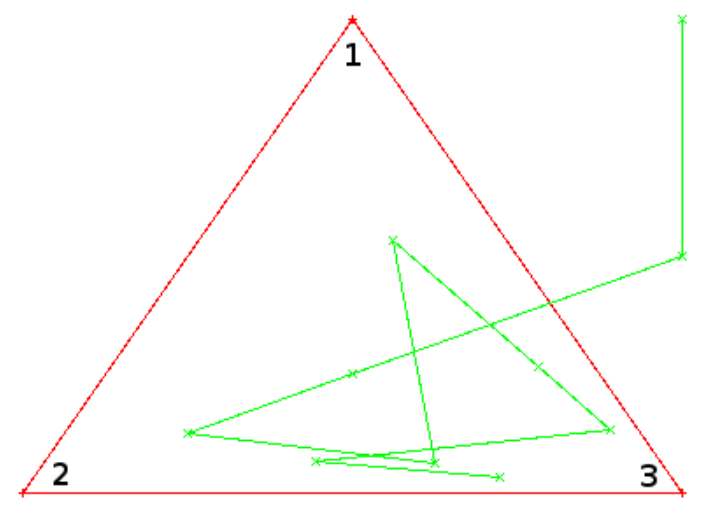

Figure 1.6: This figure shows the process that is used to generate the Sierpinski triangle. A triangle is drawn and each of the three points is labeled 1,2 and 3. Then a random point $\left.\left(x_{0}, y_{0}\right)\right)$ is selected as the initial point. A random number in the set $(1,2,3)$ is drawn. The value of $\left(x_{i}, y_{i}\right)$ is the point that is halfway between $\left(x_{i-1}, y_{i-1}\right)$ and the Nth point in the outer triangle. Note: The points are not connected by lines in the algorithm. The lines were drawn to illustrate the various iterations of the algorithm.

This random process will produce a self-similar object. Knowledge of the previous values will not help you to predict the future values. This process will always produce an object 


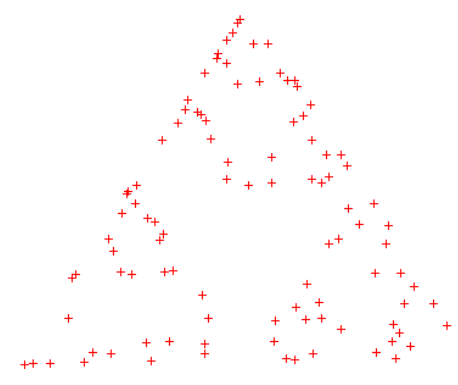

(a) 100 iterations

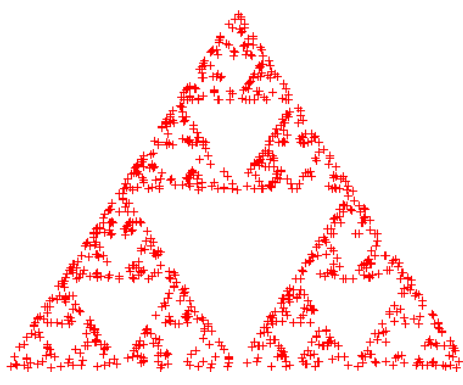

(b) 1,000 iterations

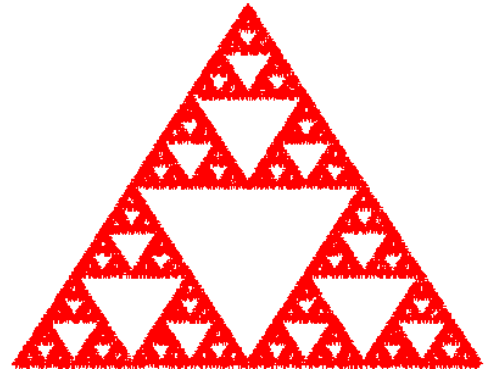

(c) 10,000 iterations

Figure 1.7: It is possible to use a random process to generate the Sierpinski triangle, which is a self-similar object.

with the same shape. There are other instances [15] where a random process can produce other self-similar objects.

\subsubsection{The Hurst parameter}

Harold Hurst [9] worked on the problem of deciding how high the Aswan Dam in Cairo, Egypt should be built. He desired to have a constant outflow of water from the dam. There should also be enough water behind the dam for the years when the water flow in the Nile River was low. Hurst studied the water flow records of the Nile river and he discovered that self similarity was present.

He came up with the Hurst parameter, which is a measure of self similarity. A pure self-similar process will have a Hurst parameter $(\mathrm{H})$ of 1 , while a random process will have a Hurst parameter of 0.5 .

Hurst's rescaled range statistic $(\mathrm{R} / \mathrm{S})$ method is fairly easy to use to compute the Hurst parameter. First the range $(\mathrm{R})$ of the values is computed: 


$$
R=\max (X)-\min (X)
$$

Then $\mathrm{R}$ is divided by the standard deviation. This will yield an equation that is proportional to the sample size raised to the power of the Hurst parameter. Specifically it is given by the following equation:

$$
\frac{R}{S} \propto N^{H}
$$

where $\mathrm{S}$ is the standard deviation of $\mathrm{X}, \mathrm{N}$ is the number of samples in $\mathrm{X}$ and $\mathrm{H}$ is the Hurst parameter. This equation can be rewritten in the following form to give the value of $\mathrm{H}$ :

$$
H=\frac{\log (R)-\log (S)}{\log (N)}
$$

\subsubsection{Self similarity in Ethernet traffic}

Leland et al. [8] did a study that found self similarity in the packet arrival times of Ethernet traffic. They found that the Hurst parameter was generally around 0.9 . The following two separate processes were influencing the traffic patterns: i) Noah Effect is data that has high variability and ii) the Joseph Effect is data that has a high long-range dependence (LRD) or self similarity. Each Ethernet source exhibits the Noah effect. It was found that aggregating multiple Ethernet sources that exhibit the Noah effect produce the Joseph effect. Figure 1.8 shows an example of aggregating multiple Ethernet sources onto a single circuit. An On Period is defined as a time period when a source/destination pair is transferring data. 
Likewise an Off Period is a time period when a source/destination pair is not transferring data.
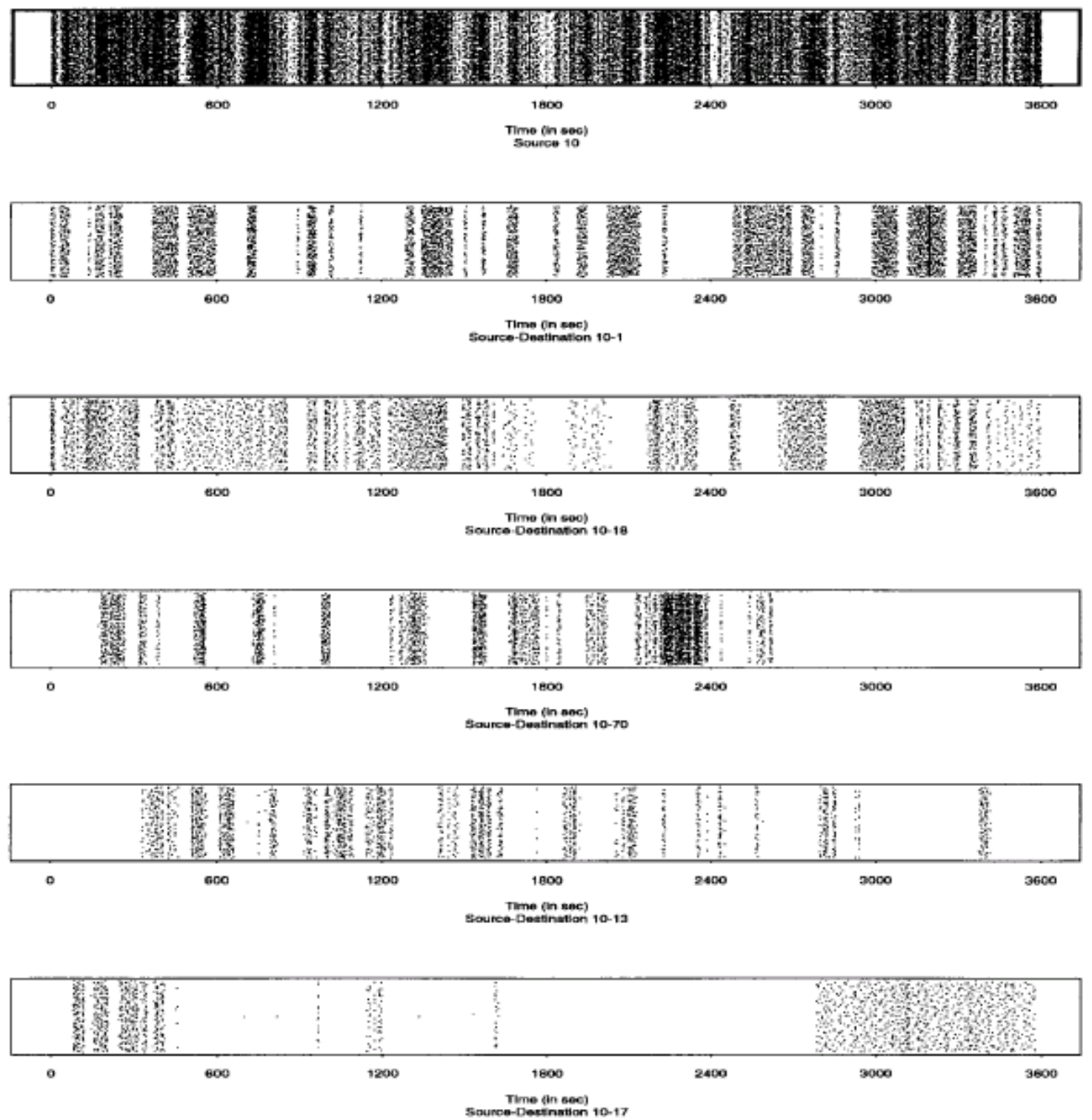

Figure 1.8: The packet arrival times for various source/destination pairs [24]. The top diagram shows the aggregated traffic for source 10. The remaining diagrams show the traffic between source 10 and some of its destinations. Each source/destination pair exhibit the Noah Effect [8], which is data that has high variability. Aggregating these source/destination pairs onto a single circuit produce the Joseph Effect [8], which is data that has a high long-range dependence (LRD) or self similarity. From [24] 


\subsubsection{The Hurst parameter and queue utilization}

The level of self similarity in network traffic can have an effect on the performance of the network [35], specifically on the queue utilization. Due to the self-similar nature of the traffic, there will be subbursts within the larger traffic bursts. The level of burstiness will be compounded as more sources are aggregated onto a single source. The compounded number of bursts can have an effect on the available buffers in the network device. Figure 1.9 shows the effect on the queue utilization as the level of self similarity increases. As the level of self similarity increases, the burstiness of the traffic will increase. This will result in higher queue utilization. This can cause delays in the network and it can have an effect on the design of high-speed networks [20].

If the Hurst parameter is close to 0.5, then aggregating multiple sources into a single stream will have less impact on the queue utilization. As the data is averaged over time, the traffic graphs will become smooth.

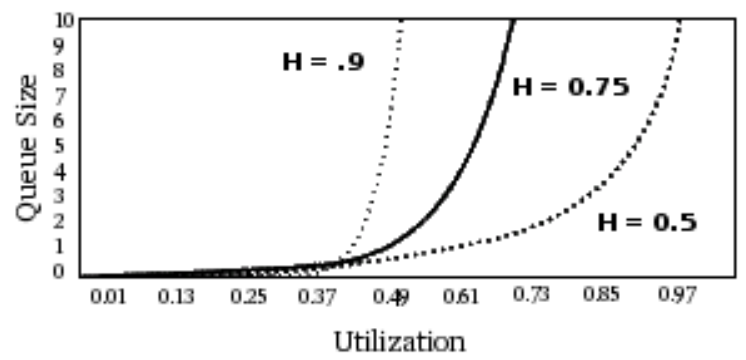

Figure 1.9: Queue utilization with different values of the Hurst parameter (H) [20], [21]. As the level of self similarity increases, the burstiness of the traffic increases as well. The increased traffic bursts have a direct impact on the queue utilization. From [21] 


\subsubsection{The auto-correlation and auto-covariance functions}

The auto-correlation function [29] is used to determine if the data came from a non-random process. The function is defined by the following equation:

$$
\frac{\sum_{i=1}^{N-k}\left(x_{i}-\mu\right)\left(x_{i-k}-\mu\right)}{\sum_{i=1}^{N}\left(x_{i}-\mu\right)^{2}}
$$

where $x_{i}$ is the $i$ th point in the time series, $\mu$ is the mean of the time series and $k$ is an integer that represents the lag. The lag is the time shift that will be examined. The values of the auto-correlation function will be in the range of $(-1,1)$, where 1 represents an exact correlation between the two time periods and -1 represents an exact anti-correlation.

The denominator of Equation 1.6 can be dropped to give the auto-covariance function:

$$
\sum_{i=1}^{N-k}\left(x_{i}-\mu\right)\left(x_{i+k}-\mu\right) .
$$

The denominator of the auto-correlation function normalizes the data so that the function will have a value in the range of $(-1,1)$.

Figure 1.10 shows the auto-covariance function between a short-range and long-range dependent process. Notice how the auto-covariance function decays hyperbolically for a long-range dependent time series. The auto-covariance function will decay exponentially for a short-range dependent time series. 


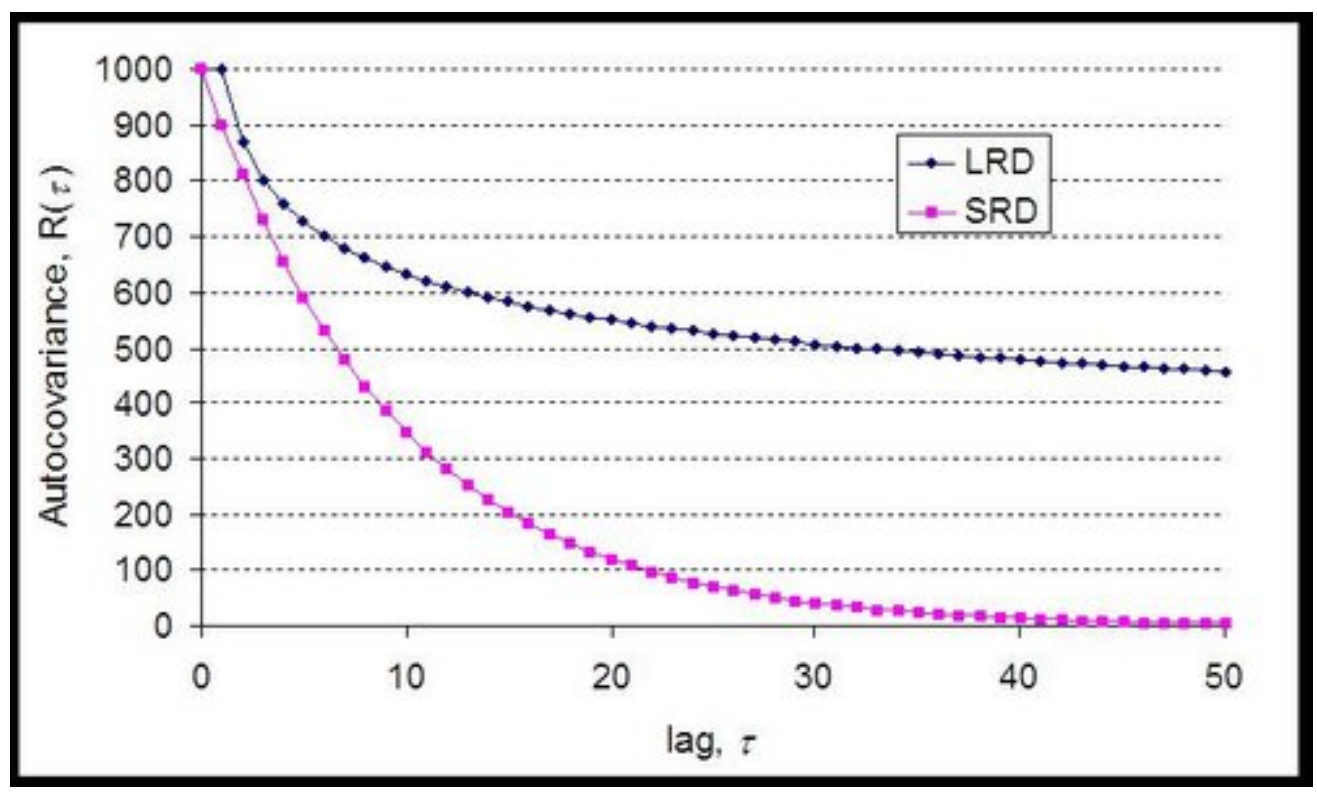

Figure 1.10: The auto-covariance function of two different processes that exhibit Short Range Dependence (SRD) and Long-Range Dependence (LRD). A SRD process will decay exponentially while a LRD process will decay hyperbolically. From [34]

\subsubsection{Long-Range dependence}

The packet arrival times of network traffic also exhibit long-range dependence [22], [5]. This occurs in heavy-tailed distributions where values that are far from the mean have a high probability. Heavy-tailed distributions are used to model packet arrival times and the length of the traffic bursts.

A distribution is said to be heavy tailed if the following equation is true:

$$
P[X>x] \sim x^{-\alpha} \text {, as } x \rightarrow \infty, 0<\alpha<2
$$

where $\alpha$ represents the degree to which the distribution may be heavy tailed. Lower values of $\alpha$ represent a distribution that has a heavier tail. If $1 \leq \alpha \leq 2$, then the distribution 
has finite mean and infinite variance. If $0<\alpha<1$, then the distribution has infinite mean and infinite variance. Figure 1.11 shows a graph of various values of alpha. Notice that as the value of $\alpha$ increases, the graph will reach it's asymptote more quickly. The graph of a heavy-tailed distribution will be hyperbolic in nature.

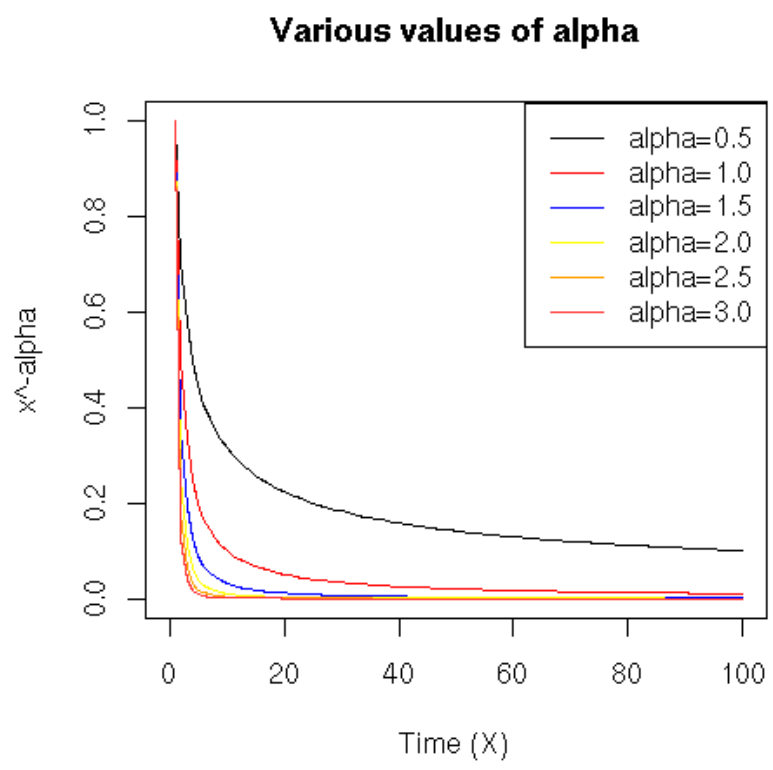

Figure 1.11: This figure shows the various values of $\alpha$. Notice that as the value of $\alpha$ increases, the graph will reach it's asymptote more quickly. The graph of a heavy-tailed distribution will be hyperbolic in nature.

Hill's estimator [16] is one technique that can be used to determine that value of $\alpha$. The value of $\alpha$ is also dependent on the Hurst parameter. It is characterized by the following equation:

$$
H=1-\frac{\alpha}{2}
$$

The terms can be rewritten in the following manner to give the value of $\alpha$ when an estimate 
of the Hurst parameter is available:

$$
\alpha=2-2 H \text {. }
$$

\subsubsection{How the data changes over time}

The amount of traffic that passes through an Internet Service Provider's network is not a static entity and it can change in many different ways. Some of the changes to the traffic can be limited to a small period of time while others can affect the traffic over a long period of time.

The amount of traffic over the short term can be influenced by one or more of the following items [13]: i) Link failures, ii) Denial of Service (DoS), iii) Flash crowds and iv) Seasonal changes (end of school year, summer break, Christmas break, weekends, etc).

The amount of traffic over the long term can be influenced by one or more of the following items [13]: i) Changes to the network topology and routing changes, ii) Changes to the number of connected customers (or their link bandwidths) and iii) New applications/protocols.

\subsection{Time series introduction}

The classical decomposition model [1] of a time series is represented by the following equation:

$$
X_{t}=m_{t}+s_{t}+Y_{t}
$$

where $m_{t}$ is the trend component, $s_{t}$ is the seasonal component and $Y_{t}$ is the random noise 
component, which is independent and identically distributed. It is possible for the trend and seasonal components to be non-existent.

\subsubsection{Decomposition using STL}

STL [4] is an algorithm that can be used to split the time series into three components: i) the trend component, ii) the seasonal component and iii) the remaining component. Figure 1.12 shows an example of the output from this algorithm.

The time series is smoothed using the LOESS algorithm [36]. This operation helps to decrease the influence of outliers in the time series. The LOESS algorithm works in the following manner: a point $(\mathrm{x})$ is chosen in the time series. The neighboring $\mathrm{q}$ points in the time series are selected as it's neighbors. Each of the neighbors is assigned weight: the weights are larger when the neighboring point is closer to the point $\mathrm{x}$. A locally fitted line of degree 1 or 2 is then drawn through these points. The LOESS algorithm is a continuous function and this ensures that the algorithm is able to properly handle missing values in the time series.

Once the time series is smoothed, it needs to be separated into the three components. There are two nested loops in the algorithm: the inner and outer loops.

The inner loop handles computing the seasonal and trend components. The time series is first detrended by subtracting the $\mathrm{k}$ previous values from the current value. Then the seasonal and trend components are calculated. Both of these components are smoothed using the LOESS algorithm and then a low-pass filter is applied.

The outer loop calls the inner loop and then it calculates the remaining component in the 
time series. The remaining component is used to calculated the robustness weights. These weights are used to reduce the influence of outliers in the time series the next time the inner loop is ran.

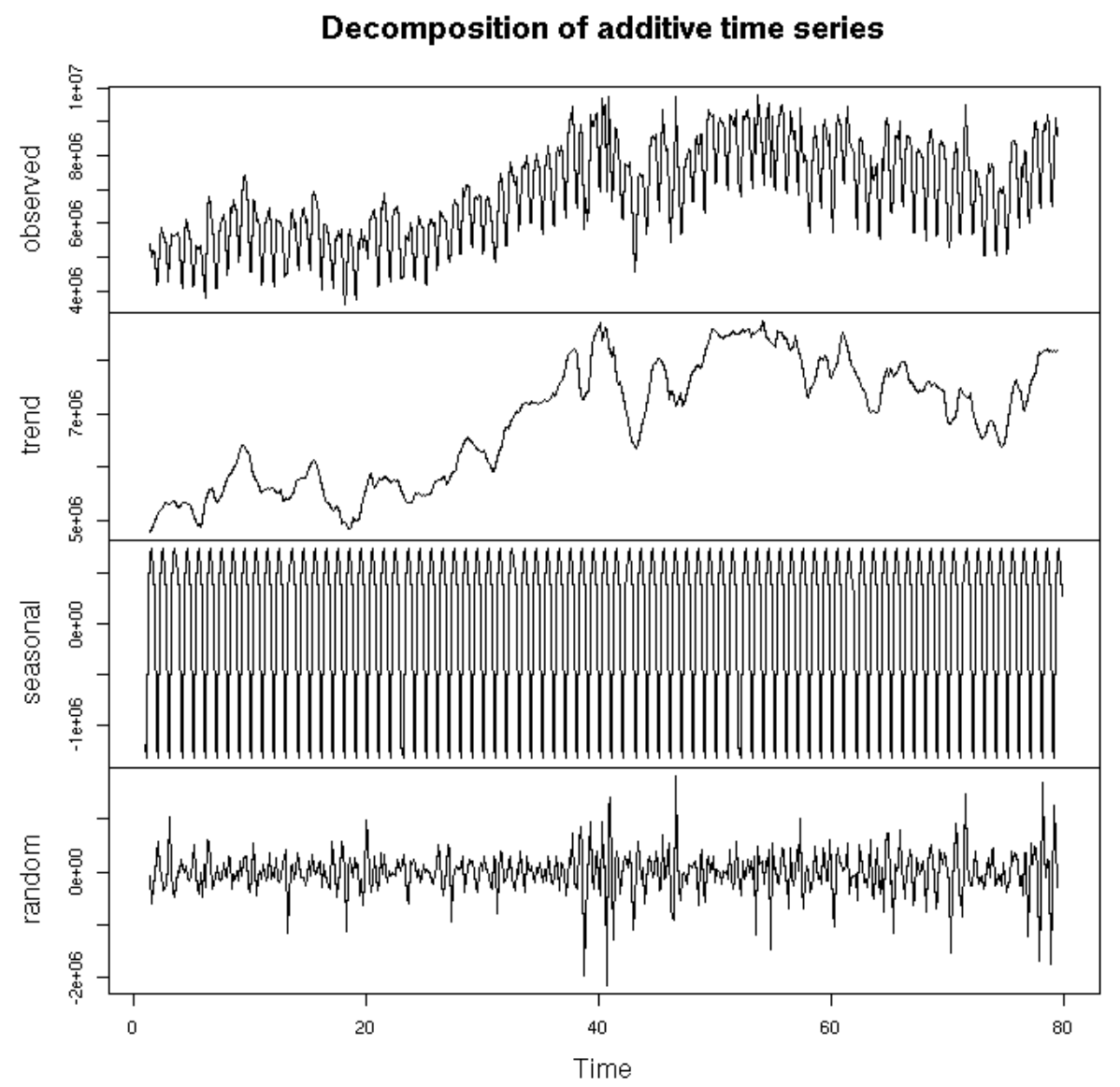

Figure 1.12: This figure shows an example of the output of the STL algorithm. This algorithm can be used to split the time series into three components: i) the trend component, ii) the seasonal component and iii) the remaining component.

\subsubsection{Stationary time series}

A time series is stationary if its statistical moments do not change over time. Specifically the time series shifted by lag $h\left(X_{1+h}, X_{2+h}, \ldots, X_{N+h}\right)$ will have the same probability distribution 
as the unshifted time series $\left(X_{1}, X_{2}, \ldots, X_{N}\right)$. This definition is usually referred to as strictly stationary since it also includes all higher-order moments. A time series is weakly stationary if its first and second order moments satisfy the definition shown above.

Observations are independent and identically distributed (iid) and have a mean of zero represent an example of a strictly stationary process. There is no relationship between the data samples. Knowledge of the previous values will not help predict the future values.

The trend component of a non-stationary time series may need to be eliminated so that the time series becomes stationary. The easiest way to do this is to apply the lag - 1 difference operator $\Delta[1]$. This operator subtracts the previous value in the time series from the current value. It is represented by the following equation:

$$
\Delta X_{t}=X_{t}-X_{t-1}
$$

This operation may need to be done on the time series multiple times until it has been determined that the time series is stationary. Generally, in practice this operation only needs to be performed one or two times [1]. Figure 1.13 shows an example of a time series where one iteration of the operation was performed.

\subsubsection{The lag operator}

The lag operator L [33] can be used to retrieve a previous value in the time series. This is mostly used to help simplify the formulas that are used to represent the time series. It is 

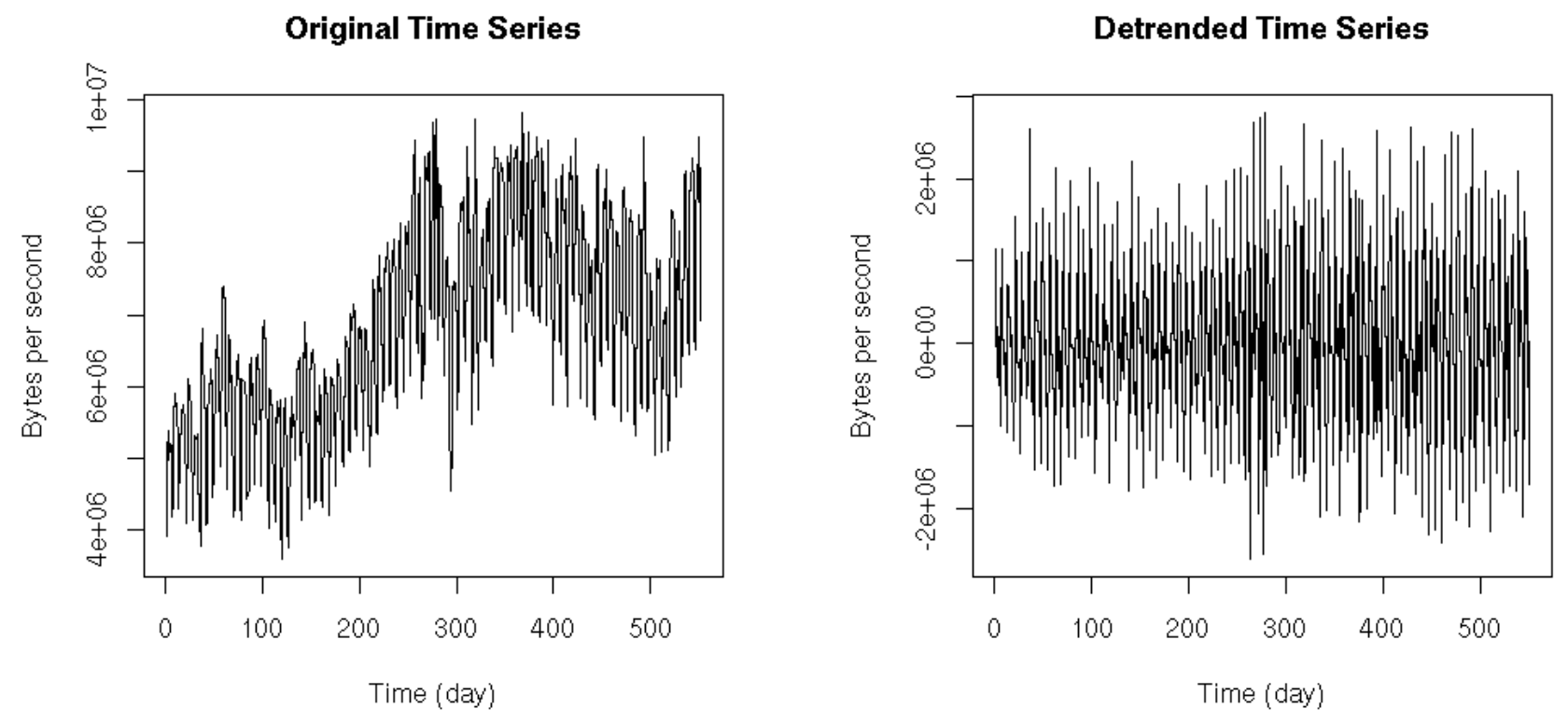

Figure 1.13: This figure shows an example of a time series where one iteration of the lag - 1 difference operator was performed. This operator subtracts the previous value in the time series from the current value. 
represented by the following equation:

$$
L^{k} X_{t}=X_{t-k}
$$

where $\mathrm{k}$ is the lag of the time series. If $\mathrm{k}$ is not specified, then it is assumed to be 1 .

\subsubsection{Seasonality}

Network traffic may also exhibit seasonality [42]. Seasonality occurs whenever there is periodic fluctuation in the data set. This can occur on the weekends and during the holidays. If there is strong seasonality in the data set, then the auto-correlation function can be used to determine the period of the season. For example: if the data set contains daily samples, then there would be a weekly seasonal component if there are fluctuations in the auto-correlation function every seven lags in the future.

There can also be multiple seasonal components in a data set [4]. For example, a data set may have a daily seasonal component and a yearly seasonal component.

\subsection{Time series models}

This section gives an introduction to the ARIMA family of functions.

\subsubsection{Autoregressive (AR)}

The formula for an autoregressive model [28] of order $\mathrm{p}(\mathrm{AR}(\mathrm{p}))$ can be represented by the following equation: 


$$
X_{t}=\sum_{i=1}^{p} \phi_{i} X_{t-i}+\epsilon_{t}
$$

where $\phi$ is a vector that represents the parameters of the model and $\epsilon_{t}$ is the error term. The value of $p$ should be less than or equal to 12 to avoid creating a degenerative model.

The autoregressive model can also be defined by the following equation using the lag operator:

$$
X_{t}=\left(\sum_{i=1}^{p} \phi_{i} L^{i}\right) X_{t}+\epsilon_{t}
$$

\subsubsection{Moving Average (MA)}

A moving average model can be used for trend estimation. The formula for a moving average model [28] of order q $(\mathrm{MA}(\mathrm{q}))$ can be represented by the following equation:

$$
X_{t}=\epsilon_{t}+\sum_{i=1}^{q} \theta_{i} \epsilon_{t-i}
$$

where $\theta$ is a vector that represents the parameters of the model and $\epsilon_{t}$ is a vector that represents the error terms. The error terms are said to be independent and identically distributed (iid) from a Gaussian distribution that have a mean of zero. The value of $q$ should be less than or equal to 12 to avoid creating a degenerative model.

The moving average model can also be defined by the following equation using the lag 
operator:

$$
X_{t}=\epsilon_{t}+\left(\sum_{i=1}^{q} \theta_{i} L^{i}\right) \epsilon_{t}
$$

\subsubsection{Autoregressive Moving Average (ARMA)}

An $\operatorname{ARMA}(p, q)$ model [28] with an autoregressive model of order $p$ and a moving average model of order q can be represented by the following equation:

$$
X_{t}=\epsilon_{t}+\sum_{i=1}^{p} \phi_{i} X_{t-i}+\sum_{i=1}^{q} \theta_{i} \epsilon_{t-i}
$$

where $\epsilon$ is a vector the represents the error terms, $\phi$ is a vector that represents the parameters of the autoregressive model and $\theta$ is a vector that represents the parameters of the moving average model. The ARMA model can also be defined by the following equation using the lag operator.

$$
\begin{aligned}
X_{t} & =\epsilon_{t}+\left(\sum_{i=1}^{p} \phi_{i} L^{i}\right) X_{t}+\left(\sum_{i=1}^{q} \theta_{i} L^{i}\right) \epsilon_{t} \\
\left(1-\sum_{i=1}^{p} \phi_{i} L^{i}\right) X_{t} & =\left(1+\sum_{i=1}^{q} \theta_{i} L^{i}\right) \epsilon_{t} .
\end{aligned}
$$




\subsubsection{Autoregressive Integrated Moving Average (ARIMA)}

The ARIMA(p,d,q) model is used when the data exhibits trend. Once the trend is eliminated, it will reduce to a ARMA model. The $\mathrm{p}$ and $\mathrm{q}$ parameters are the same parameters that are used in an $\operatorname{ARMA}(\mathrm{p}, \mathrm{q})$ model. The $\mathrm{d}$ parameter represents the finite number of times to difference the time series. The differencing operation is used to remove the trend component from the time series. Typically d has a value that is less than or equal to 2.

The ARIMA model can be represented by the following equation using the lag operator:

$$
\left(1-\sum_{i=1}^{p} \phi_{i} L^{i}\right)(1-L)^{d} X_{t}=\left(1+\sum_{i=1}^{q} \theta_{i} L^{i}\right) \epsilon_{t}
$$

It should be noted that $\operatorname{ARIMA}(p, 0, q)=\operatorname{ARMA}(p, q), \operatorname{ARIMA}(p, 0,0)=\operatorname{AR}(p)$ and $\operatorname{ARIMA}(0,0, q)$ $=\operatorname{MA}(q)$.

\subsubsection{Fractionally Integrated ARMA (FARIMA)}

A fractionally integrated ARMA process is an ARIMA process that has a value of $d$ in the range of $-0.5 \leq d \leq 0.5$. A FARIMA model is used whenever the data exhibits self similarity. The value of $d$ can be calculated by the following equation:

$$
d=H-0.5
$$

where $\mathrm{H}$ represents the Hurst parameter of the time series. 


\subsection{6 $\phi$ and $\theta$ parameter calculation}

The smallest possible values of $\mathrm{p}$ and $\mathrm{q}$ should be used that will adequately model the time series. Whenever $\mathrm{p}$ and $\mathrm{q}$ have been chosen, least squares can be used to determine the proper values of $\phi$ and $\theta$. Least squares will fit a line or a curve, $\mathrm{f}(\mathrm{x})$, to the data set. The function, $\mathrm{f}(\mathrm{x})$, that minimizes the following equation is chosen:

$$
L=\sum_{i=1}^{n}\left(Y_{i}-f\left(X_{i}\right)\right)^{2}
$$

where $f\left(X_{i}\right)$ is a function that represents the fitted line or curve and $Y_{i}$ is the original point in the data set.

\subsubsection{ARIMA prediction}

In order to predict the future values in a time series, an appropriate model has to be built that represents the time series. The generated model is then used to predict the future values in the time series.

The data set needs to be split into two distinct sets: a training set and a validation set. The training set is used to build the ARIMA model. The generated ARIMA model is compared with the validation set to see how the model fits the data. Once a suitable model has been found, the model can be used to predict the future values in the time series.

Over time, the characteristics of the time series may change. This may cause the generated model to be incorrect. When this occurs, the model will need to be retrained so that it will obtain better results. 


\subsection{Short-Term bandwidth estimation}

Short-term bandwidth estimation algorithms will forecast the traffic utilization seconds or minutes into the future. This can be used for quality of service (QoS) algorithms in timesensitive applications such as multimedia. Short-term bandwidth estimation can also be used to determine if the current observed value of the traffic is outside of what is considered to be normal. RRDTool [40] has a feature called Aberrant Behavior Detection [2] which uses the Holt-Winters forecasting algorithm [1] to see if the observed value should raise an alarm to the system administrator.

\subsection{Long-Term bandwidth estimation}

Long-term bandwidth estimation deals with forecasting the amount of bandwidth usage several months into the future. [13] is the only paper that was found that deals directly with the long-term forecasting of IP network traffic utilization.

\subsubsection{Long-Term forecasting using wavelets}

The authors of [13] claim their technique can predict the traffic utilization six months in the future with a 10-15\% error (Figure 1.14). They generate a model for each circuit since each circuit will have its own traffic patterns.

They averaged the data into 90 minute intervals and used wavelets [23] to break the signal into its basis functions. They used the sixth wavelet resolution as the coarsest time scale, which corresponds to the 96 -hour time period $\left(2^{6} \cdot 1.5\right)$. They found that $98 \%$ of the total 


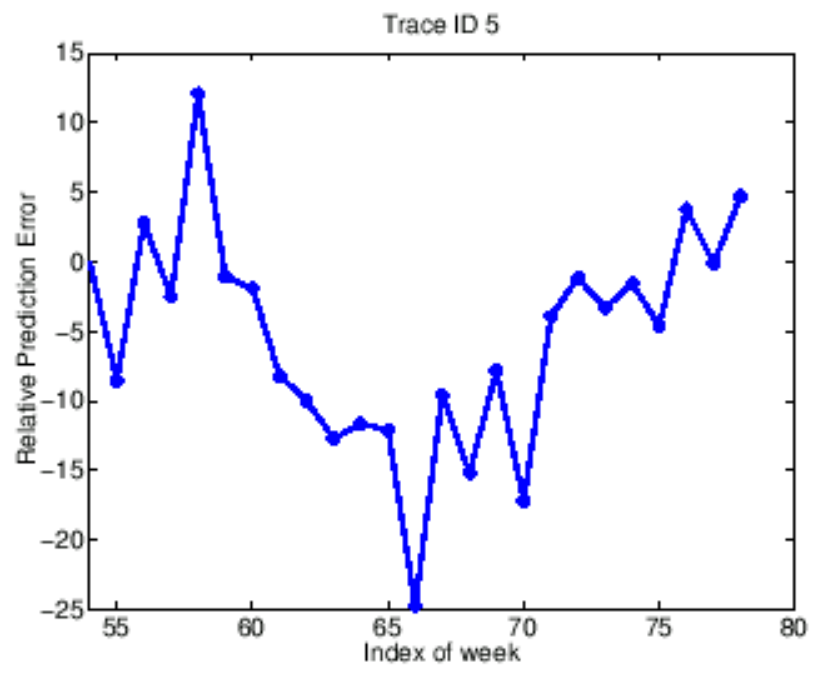

Figure 1.14: The traffic prediction error using Long-Term Forecasting with wavelets. From [13]

energy comes from the following two items: i) the long-term trend (sixth wavelet resolution) and ii) fluctuations at the 12-hour time scale (third wavelet resolution). Using Analysis of Variance (ANOVA) [27], these two items explain $90 \%$ of the variance.

The Fast Fourier Transform (FFT) shows strong periods at the 12 and 24-hour time scale (See Figure 1.15). The maximum amount of energy was always in the third wavelet time period, which corresponds to the 12 -hour time scale $\left(2^{3} \cdot 1.5=12\right)$. This time scale accounts for $97-99 \%$ of the total energy. The next highest amount of energy was in the fourth wavelet time period, which corresponds to the 24 -hour time scale. The first, second, fifth and sixth resolutions only contributed less than $5 \%$ of the total variance in the detail signals.

Using wavelets, the entire signal can be reconstructed using the following equation:

$$
x(t)=c_{p}(t)+\sum_{j=1}^{p} d_{j}(t)
$$



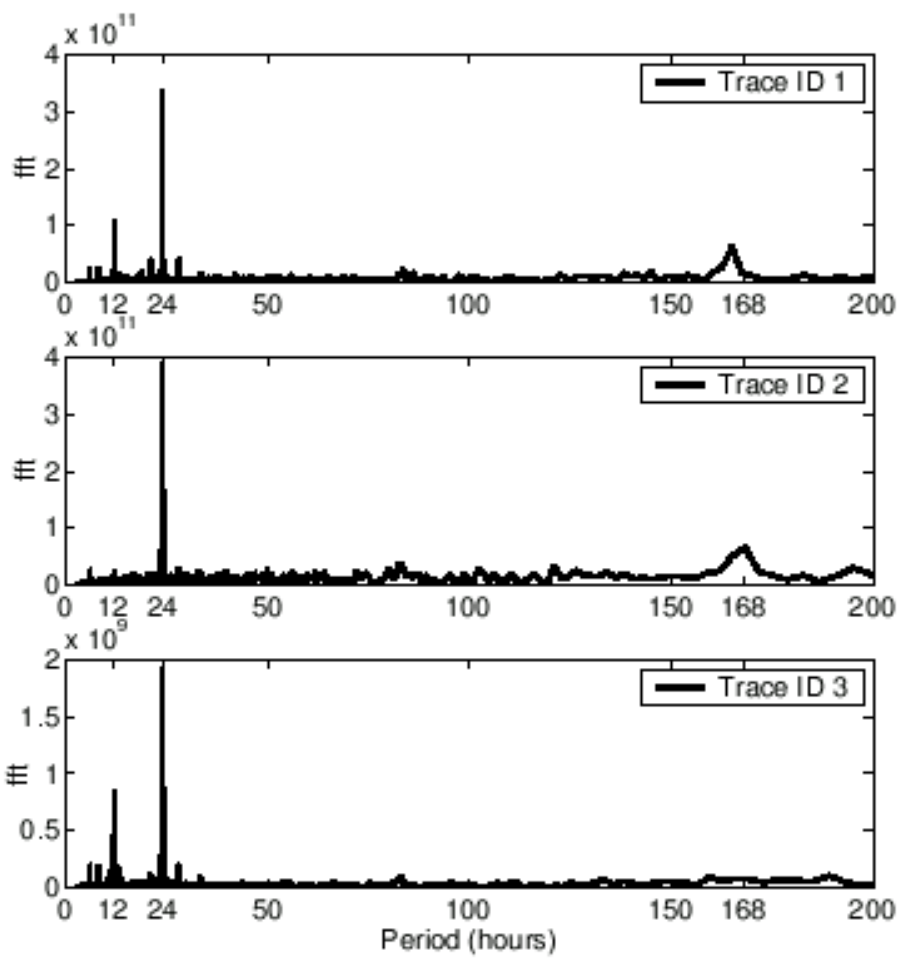

Figure 1.15: The Fast Fourier Transform (FFT) of three network traces. There are strong periods at the 12 and 24-hour time scale. From [13]

where $p$ is the wavelet resolution (number of layers), $c_{p}(t)$ is the a-trous wavelet transformation, and $d_{j}(t)=c_{j-1}(t)-c_{j}(t)$.

The a-trous wavelet transformation means "with holes" [25], [26]. Figure 1.16 shows the terms that are used in the expansion of $c_{3}(5)$. The coarser wavelet resolutions will require the values from a wider range of time that will help to produce a smoother signal.

The forecasting algorithm was simplified to the following equation:

$$
x(t)=c_{6}(t)+\beta d_{3}(t)
$$

where $c_{6}(t)$ is the overall long-term trend (recall, the sixth wavelet resolution). They found 


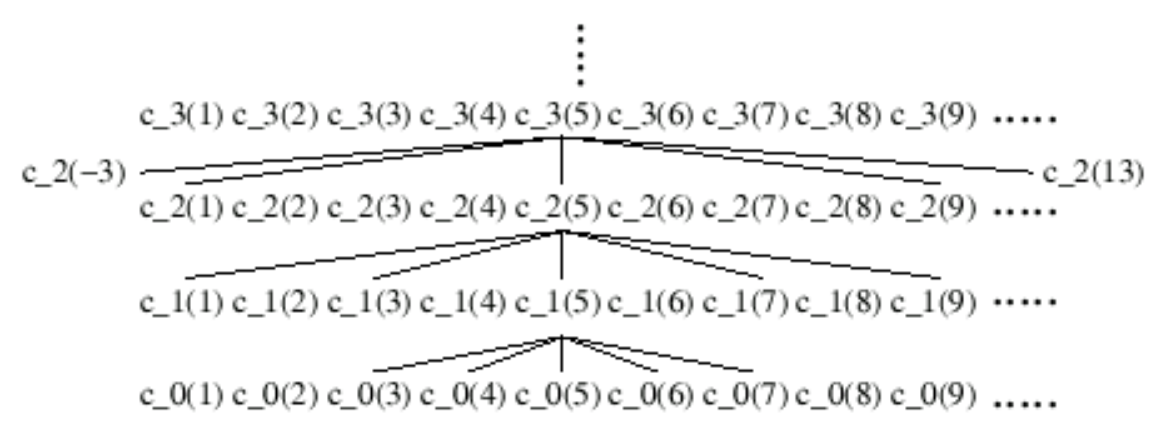

Figure 1.16: The terms that are used in the expansion of $c_{3}(5)$ in the a-trous wavelet transformation [13]. The coarser wavelet resolutions will require the values from a wider range of time which will help to produce a smoother signal. From [13]

that setting $\beta$ to 3 gave good results. The prediction results for one of their traffic traces are shown in Figure 1.17.

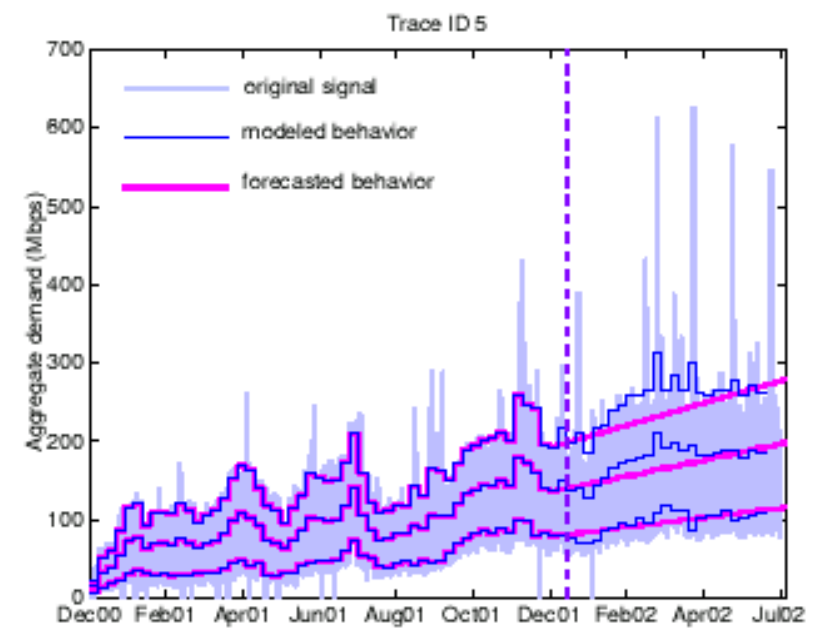

Figure 1.17: The six-month prediction of the traffic. From [13]

\subsection{Principal Component Analysis (PCA)}

Principal Component Analysis (PCA) [6] seeks to project a d dimensional data set onto a lower dimensional $\mathrm{k}$ subspace. It accomplishes this by rotating the coordinate system so 
that the variance is maximized across each dimension. This will decorrelate the data. The dimensions that have a low variance can be eliminated. Figure 1.18 shows an example of how the coordinate system may be rotated using PCA.

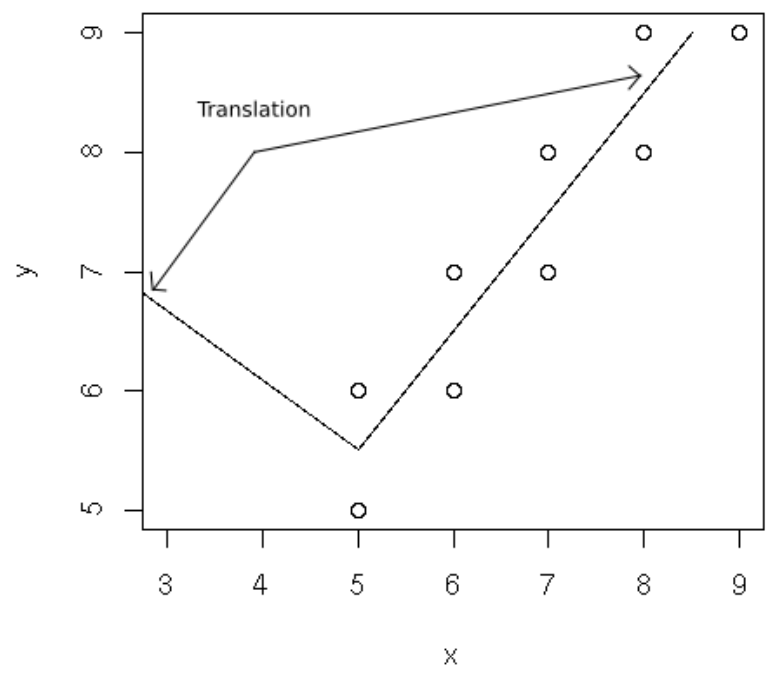

Figure 1.18: Principal Component Analysis (PCA) will rotate the coordinate system so that the variance is maximized across each dimension. This figure shows an example of how the coordinate system may be rotated using PCA.

The mean $(\mu)$ and covariance matrix $(\Sigma)$ is calculated for the entire data set. Then the eigenvalues and corresponding eigenvectors are computed for each dimension. The list of eigenvectors and eigenvalues are sorted in decreasing order by their associated eigenvalue. The first few eigenvalues will typically be very large while the later ones will generally be small. The eigenvectors whose eigenvalues represent the top $95 \%$ or $99 \%$ of the total variance are typically kept. The reduced eigenvectors are used to project the data onto a lower $\mathrm{k}$ dimensional subspace using the following equation: 


$$
Y=A^{t}(X-\mu)
$$

where $\mathrm{A}$ is a matrix that contains the reduced number of eigenvectors, $\mathrm{X}$ is the original signal and $\mu$ is the mean of $\mathrm{X}$.

PCA is a filtering method that can be used to remove the noise in a data set. This assumes the real signal will always be captured in the top eigenvectors and the noise will be in the remaining eigenvectors. This may not always be the case. In some cases, the noise can be in the first few eigenvectors while the signal is in the remaining eigenvectors. In cases like this, the top eigenvectors should be removed.

Once the number of eigenvectors has been reduced, the data can be transformed into the original coordinate system by using the following equation:

$$
X^{\prime}=Y \cdot E+\mu
$$

where $\mathrm{Y}$ is the projected data, $\mathrm{E}$ is the transformation (or rotation) matrix and $\mu$ is the mean of the original data set $(\mathrm{X})$.

\subsection{Independent Component Analysis (ICA)}

Independent Component Analysis (ICA) [6] seeks to find the underlying components in a data set that are the most independent of each other. ICA transforms the data set so that the yth order statistics are optimized. Figure 1.19 shows an example of how the data set may be transformed using ICA. 


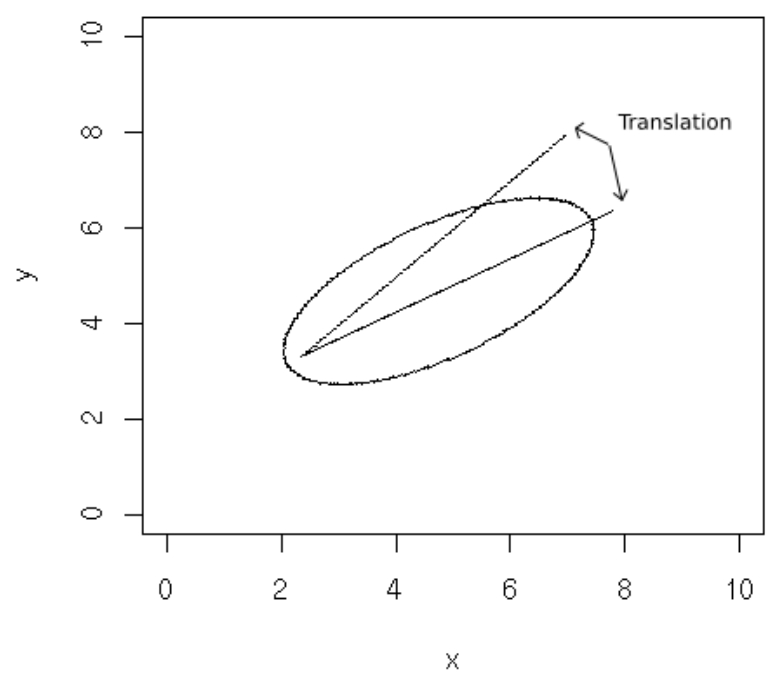

Figure 1.19: Independent Component Analysis (ICA) transform the data set so that the yth order statistics are optimized. This figure shows an example of how the data set may be transformed using ICA.

The cocktail party problem [31] is an excellent example that illustrates the concept behind ICA. Let's say that there are N simultaneous conversations in a room. You also have $\mathrm{N}$ devices recording those conversations. ICA can be used to isolate the individual conversations.

Figure 1.20 shows the the concept behind ICA. ICA assumes that there are two or more independent source signals that are mixed linearly by the mixing matrix A in some unknown fashion to form one or more mixed signals. The signals are mixed by the following equation:

$$
Y=A \cdot X
$$

where $\mathrm{A}$ is the mixing matrix and $\mathrm{X}$ contains the original signals. To determine the in- 


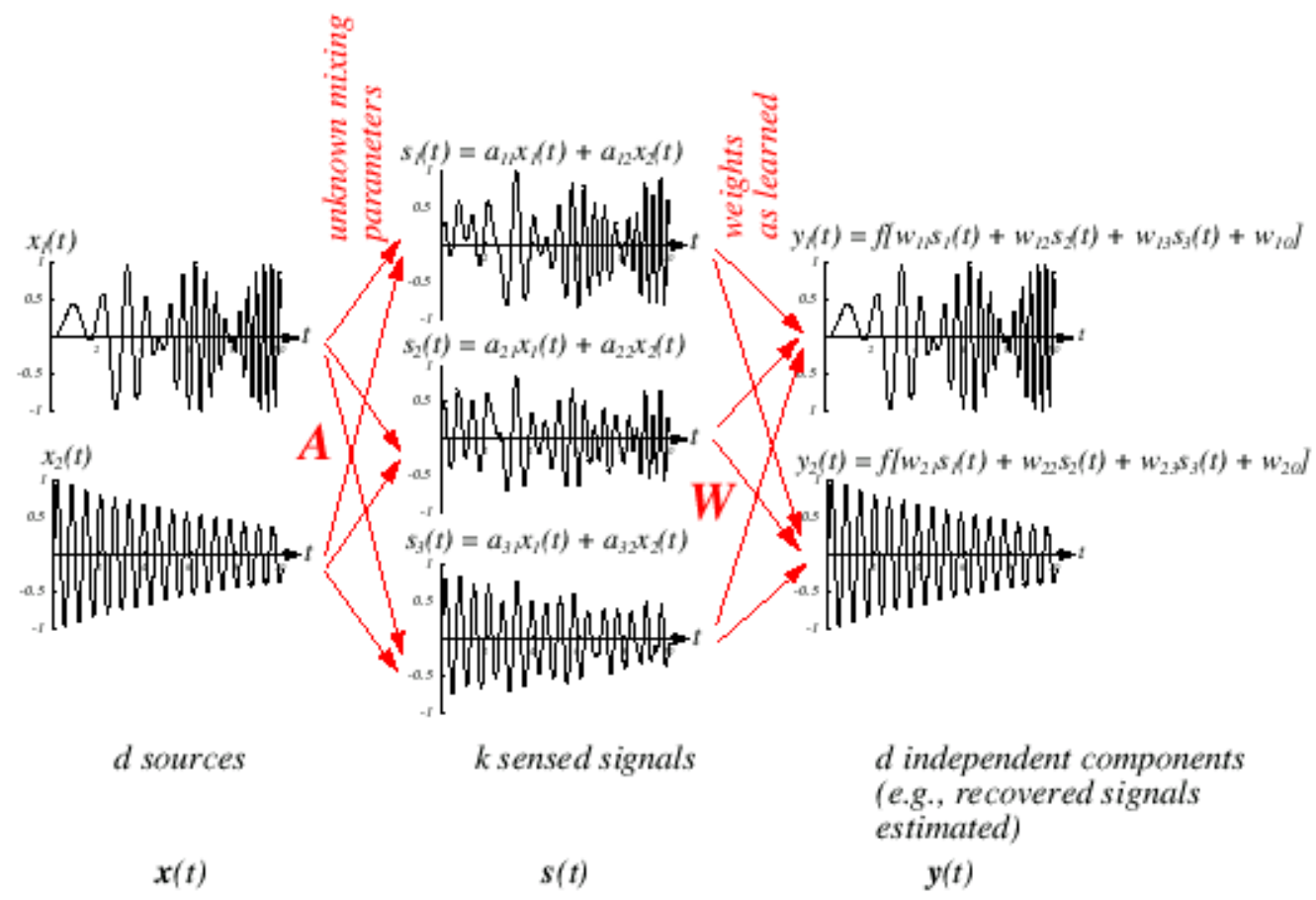

Figure 1.20: ICA assumes that there are two or more independent source signals, $\mathbf{x}(t)$, that are mixed linearly in some unknown fashion (A) to form one or more mixed signals, $\mathbf{s}(t)$. To determine the independent components $(\mathbf{y}(t))$, the inverse mixing matrix $(\mathbf{W})$ needs to be calculated that gives the components that are the most independent of each other. From [6]

dependent components, the inverse mixing matrix (W) needs to be calculated that gives the components that are the most independent of each other. The original signal can be reconstructed by using the following equation:

$$
X^{\prime}=Y \cdot W
$$

where $\mathrm{Y}$ is the projected data and $\mathrm{W}$ is the inverse mixing matrix. 


\section{INTRODUCTION TO THE DATA SETS}

\subsection{Data set information}

There were six data sets that were analyzed in this thesis. These data sets came from a regional Internet Service Provider's network. Due to the sensitive nature of the data, the ISPs name along with a detailed description of each data set cannot be released.

Two of the data sets came from large circuits (DS3) while the remaining four data sets came from smaller circuits (T1). Each data set contains between 1.5 to 2 years worth of daily averages.

\subsubsection{Data polling}

The inbound and outbound traffic counts were retrieved using the SNMP [43] protocol every 5 minutes. Each interface in the network was not polled at the same time. Instead, each interface was polled serially. Specifically the ifTable or ifXTable MIBs from RFC1213/RFC2233 [30] were polled on all of the routers. The ifTable MIB represents the 32-bit counters while the ifXTable MIB represents the 64-bit counters. The 64-bit MIBs are not supported on interfaces that have a speed less than 20Mbit. Also, the 32-bit MIBs could not be used for the larger circuits since the 32-bit counters would overflow frequently.

It should be noted that the SNMP protocol uses the User Datagram Protocol (UDP) 
[37]. This is an unreliable protocol. There were small time periods that could have unknown values. These unknown values are flushed from the database whenever the consolidated points were generated in the database.

\subsubsection{Data set statistics}

Figure 2.1 shows a graphical representation of the daily and weekly averages of the data sets.

Table 2.1 shows some numerical statistics about the data.

\begin{tabular}{|c|c|c|r|r|r|r|}
\hline Data Set \# & \# Daily Samples & Traffic In/Out & Min & Max & Mean & Std Dev \\
\hline 0 & 555 & In & 3599349.74 & 9811449.16 & 6874157.41 & 1471047.24 \\
0 & 555 & Out & 1361942.28 & 3095551.69 & 2123761.77 & 294614.89 \\
1 & 590 & In & 7.65 & 368239.76 & 64546.30 & 41706.94 \\
1 & 590 & Out & 5.45 & 297067.59 & 38432.36 & 39843.43 \\
2 & 737 & In & 138.04 & 128465.44 & 38260.99 & 30960.18 \\
2 & 737 & Out & 0 & 593730.20 & 57893.48 & 80717.47 \\
3 & 737 & In & 0 & 283122.09 & 45617.11 & 37830.64 \\
3 & 737 & Out & 8.50 & 651883.67 & 188043.16 & 143546.89 \\
4 & 737 & In & 0 & 135552.09 & 38479.68 & 21485.99 \\
4 & 737 & Out & 0 & 86491.08 & 22496.22 & 14986.19 \\
5 & 544 & In & 3225109.64 & 9922605.91 & 6193920.10 & 1468120.15 \\
5 & 544 & Out & 2606407.96 & 9443537.14 & 6140173.40 & 1567154.49 \\
\hline
\end{tabular}

Table 2.1: The numeric representation of the six data sets that were analyzed in this paper.

\subsection{Introduction to RRDTool}

RRDTool [40] is a front-end to the Round-Robin Database (RRD). RRD databases are designed to efficiently store and graph time series data sources. RRDWorld [41] shows a list of several software packages that currently use RRDTool.

The size of a RRD database is a constant size so that it will not grow over time. The values in the time series will be transparently combined with nearby samples based on the 


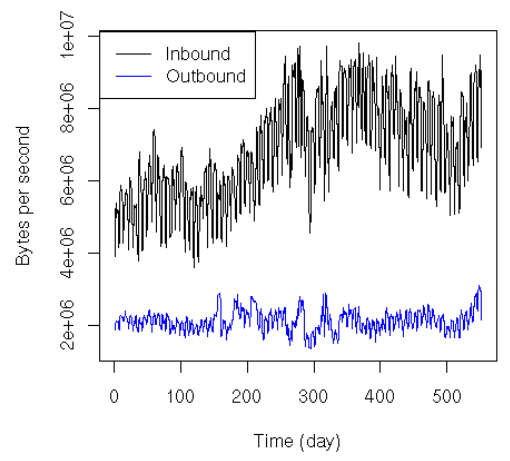

(a) Data Set \#0

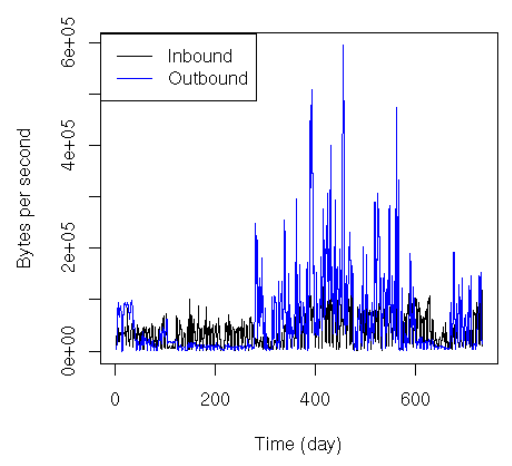

(c) Data Set \#2

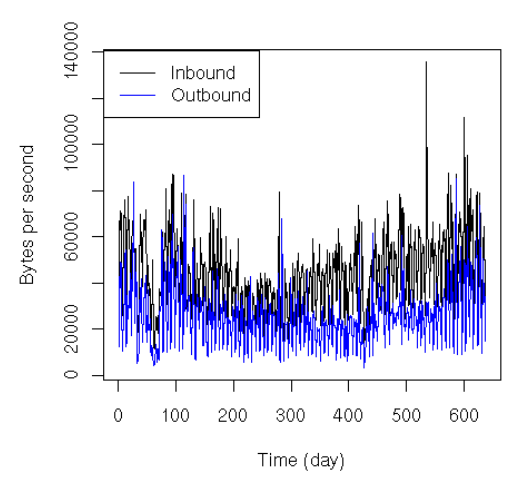

(e) Data Set \#4

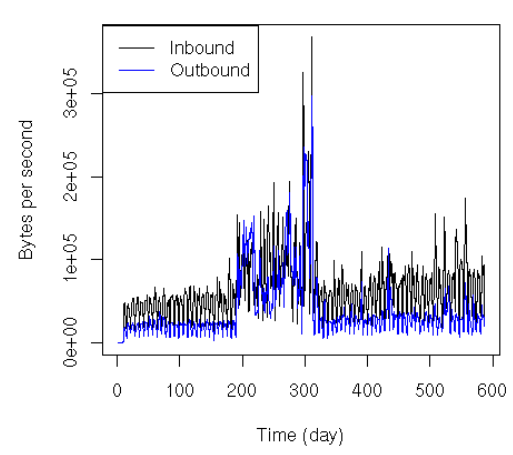

(b) Data Set \#1

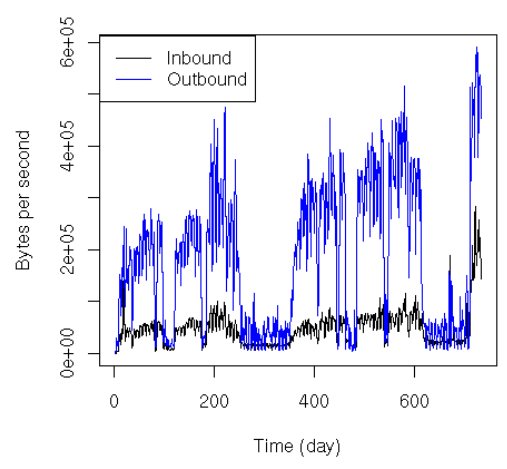

(d) Data Set \#3

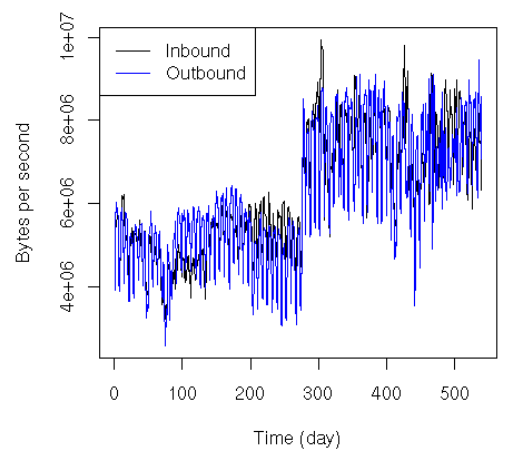

(f) Data Set \#5

Figure 2.1: The graphs of the six data sets that were analyzed in this paper. 
parameters of the database.

\subsection{RRD database parameters}

A RRD database consists of two parts: i) one or more data source definitions (DS) and ii) one or more Round-Robin Archives (RRA). A DS represents a measurement that is stored in the database. There are typically two separate data sources when network traffic utilization is stored in a RRD database: i) the current amount of inbound traffic and ii) the current amount of outbound traffic. A RRA represents how the data should be stored in the database along with the rules for how the data should be consolidated. Tables 2.2 and 2.3 shows the DS and RRA parameters of the RRD databases that were analyzed in this paper. The meaning of these fields are shown below. The rrdcreate(1) [39] man page should be consulted for a detailed explanation of these fields.

\subsubsection{Data Source (DS) parameters}

\begin{tabular}{|c|c|c|c|c|}
\hline Data Source Name & Data Source Type & Heartbeat & Minimum & Maximum \\
\hline traffic in & Counter & 900 & 0 & $\mathrm{U}$ \\
traffic_out & Counter & 900 & 0 & $\mathrm{U}$ \\
\hline
\end{tabular}

Table 2.2: The Data Source (DS) parameters of the RRD database.

The Data Source Type can be one of the following types: i) COUNTER, ii) DERIVE, ii) ABSOLUTE and iv) GAUGE. A counter represents a data source whose value monotonically increases with time (except for overflows). This is typically how traffic on a router is represented. Derive will store the rate of change between the last data sample and the current data sample. Absolute represents a data source whose counter is reset after the value 
is read. Gauge represents a data source where the current value that is read does not need to be changed.

The minimum and maximum fields represent the bounds of the data that can be stored in the database. This can be useful to eliminate erroneous values due to incorrect measurements. A maximum value of $\mathrm{U}$ specifies that there is no ceiling to the data.

\subsubsection{Round-Robin Archive (RRA) parameters}

\begin{tabular}{|c|c|c|c|c|}
\hline Consolidation Function & X Files Factor & Steps $(s)$ & Rows $(r)$ & Time period $(t)$ \\
\hline Average & 0.5 & 1 & 600 & 2.08 days \\
Average & 0.5 & 6 & 700 & 14.58 days \\
Average & 0.5 & 24 & 775 & 64.58 days \\
Average & 0.5 & 288 & 797 & 797 days \\
Maximum & 0.5 & 1 & 600 & 2.08 days \\
Maximum & 0.5 & 6 & 700 & 14.58 days \\
Maximum & 0.5 & 24 & 775 & 64.58 days \\
Maximum & 0.5 & 288 & 797 & 797 days \\
\hline
\end{tabular}

Table 2.3: The Round-Robin Archive (RRA) parameters of the RRD database.

Recall that the Round-Robin Archive (RRA) is the section of the database where the data will be stored. The Consolidation Function specifies how to combine multiple data samples into a single point. The following functions are available: i) AVERAGE, ii) MIN, iii) MAX and iv) LAST. The steps parameter defines how many data samples are used to generate a consolidated data sample. The rows parameter defines how many consolidated data sample entries are available in the RRA.

The $X$ Files Factor parameter requires a little bit more explanation. There are times when the process that is collecting the source data is unable to retrieve the value (or update the database). In these cases RRDTool needs to mark which time periods have unknown 
time values. When a new consolidated point is being generated, the X Files Factor parameter is a percentage that represents how much of the primary data samples have to be unknown before the consolidated data sample is marked as unknown.

\subsubsection{Time period calculation}

The following equation can be used to calculate the time period that the RRA will cover:

$$
t=p \cdot s \cdot r
$$

where $\mathrm{p}$ is the step size, $\mathrm{s}$ is the number of steps, $\mathrm{r}$ is the number of rows and $\mathrm{t}$ is the time period that is covered. The step size specifies how often the database is updated with a new value. The step size is 5 minutes in this data set. The computation for the total time period in the first RRA shown in Figure 2.3 is $5 \cdot 1 \cdot 600=3000$ minutes or 2.08 days.

\subsection{Data set analysis}

\subsubsection{Auto-correlation function}

Figure 2.2 shows the auto-correlation function of the six data sets. This figure was visually compared with the data set graphs. It was noted that higher amounts of traffic on a circuit corresponded to an auto-correlation function that does not decay exponentially. It is possible that these data sets exhibit long-range dependence. 


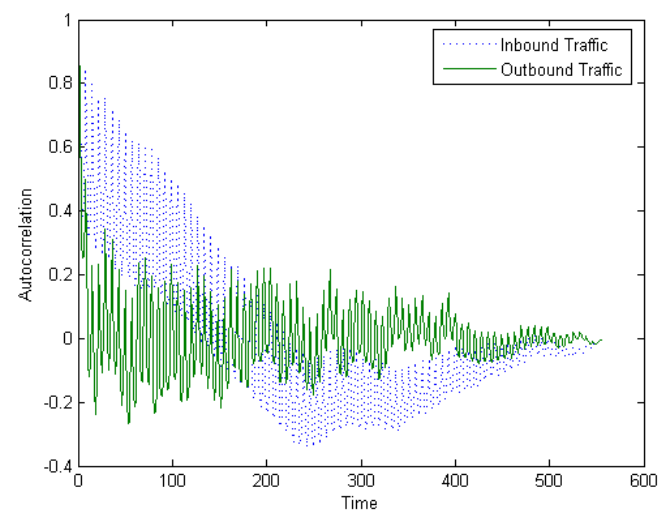

(a) Data Set \#0

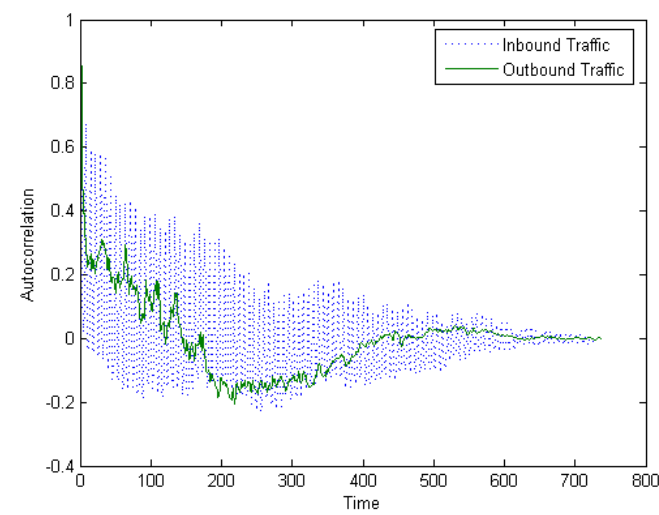

(c) Data Set \#2

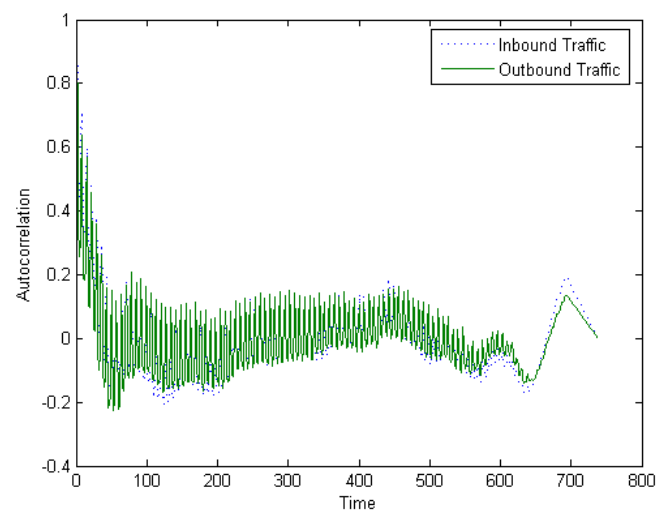

(e) Data Set \#4

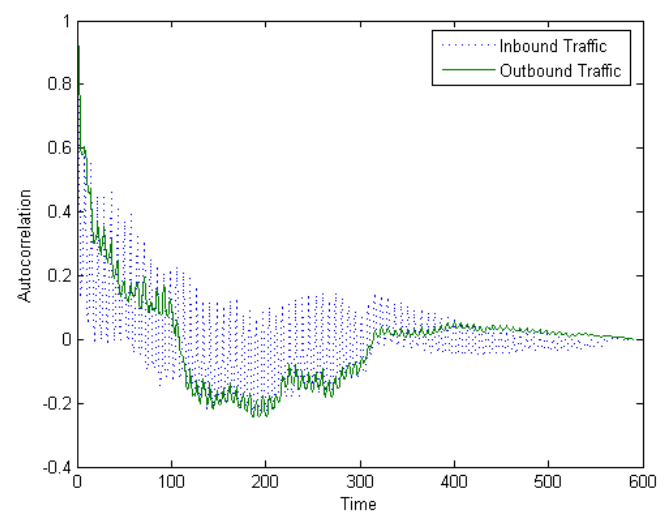

(b) Data Set \#1

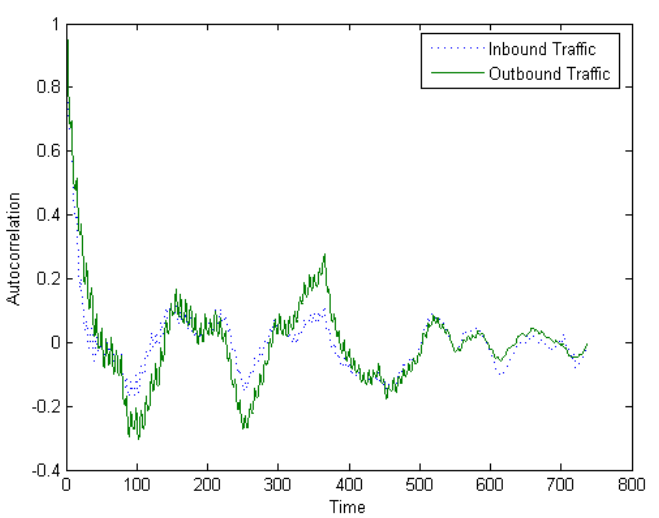

(d) Data Set \#3

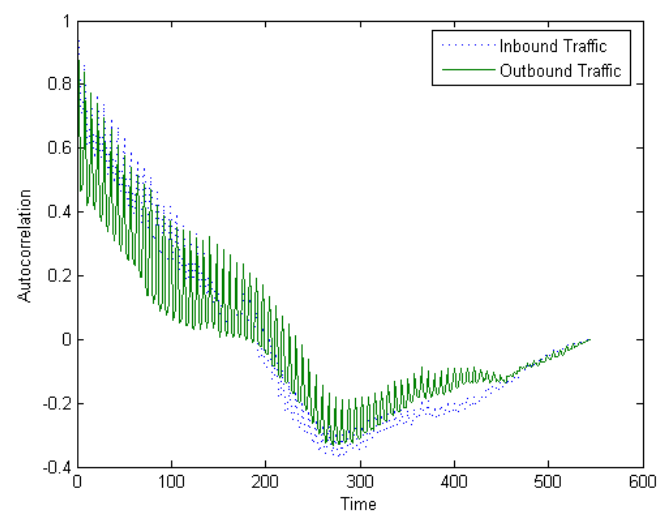

(f) Data Set \#5

Figure 2.2: The auto-correlation function of the six data sets, time in days. 


\subsubsection{Self-Similarity test}

To verify that the data sets exhibit self similarity, the Hurst parameter was calculated for the inbound and outbound traffic on all six data sets. Figure 2.4 shows the calculated Hurst parameters. All six data sets exhibit some degree of self similarity.

\begin{tabular}{|c|c|c|c|c|c|c|}
\hline & \multicolumn{3}{|c|}{ Inbound Hurst } & \multicolumn{3}{c|}{ Outbound Hurst } \\
\hline Data Set \# & R/S Method & VTP Method & IDC Method & R/S Method & VTP Method & IDC Method \\
\hline 0 & 0.82743 & 0.98800 & 0.98801 & 0.63420 & 0.49617 & 0.49618 \\
1 & 0.74591 & 0.87659 & 0.87659 & 0.81124 & 0.84108 & 0.84108 \\
2 & 0.76234 & 0.86612 & 0.86610 & 0.78543 & 0.85148 & 0.85142 \\
3 & 0.70500 & 0.66503 & 0.66539 & 0.76869 & 0.77622 & 0.77648 \\
4 & 0.77138 & 0.78095 & 0.78052 & 0.74328 & 0.73095 & 0.73055 \\
5 & 0.85928 & 0.97878 & 0.97878 & 0.84440 & 0.98374 & 0.98373 \\
\hline
\end{tabular}

Table 2.4: The Hurst parameters for the six data sets. Three methods were used to calculate the Hurst parameter: $i)$ the rescaled range statistic $(R / S)$ method, ii) the variance time plot method and iii) the index of dispersion for counts

\subsubsection{Test for seasonality}

It was also noted that the auto-correlation function had large fluctuations throughout the graph. This is most likely caused by a seasonal component in the data sets. Each row in the data sets were separated into its corresponding day of the week. The inbound and outbound traffic plots by the day of the week are shown in Figure 2.3, Figure 2.4 and Table 2.5. These graphs show that there is a possible seasonal component to these data sets. Specifically there is a noticeable decline in the traffic utilization on Saturday and Sunday.

T-tests were performed on these data sets to determine if the observed differences between each day of the week was significant. Tables 2.6 and 2.7 show the p-values for each day of the week in the six data sets. The data shows that there is a seasonal component on the 


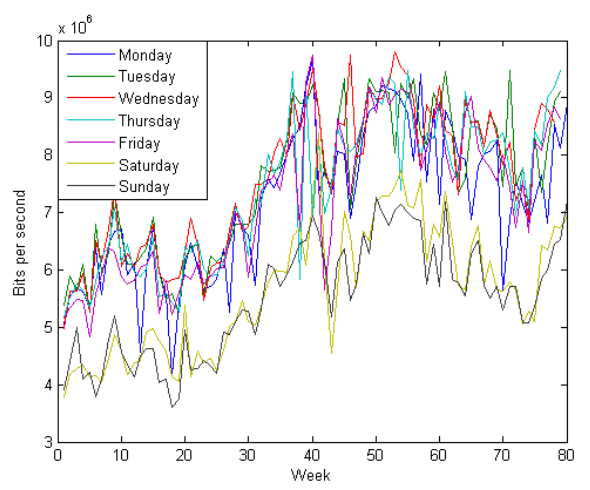

(a) Data Set \#0

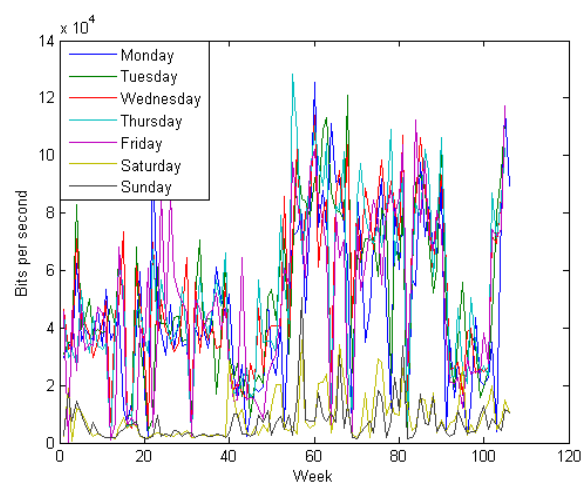

(c) Data Set \#2

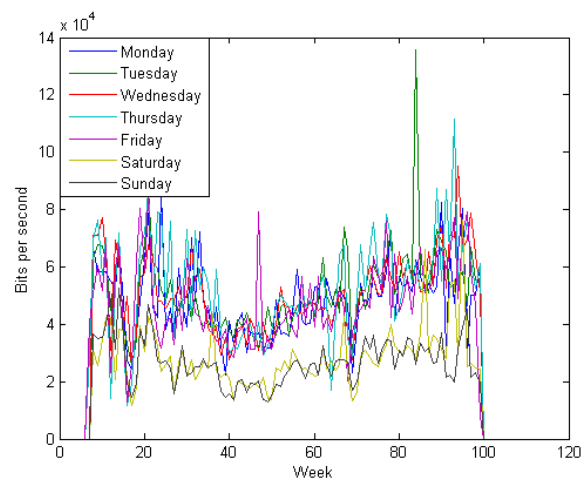

(e) Data Set \#4

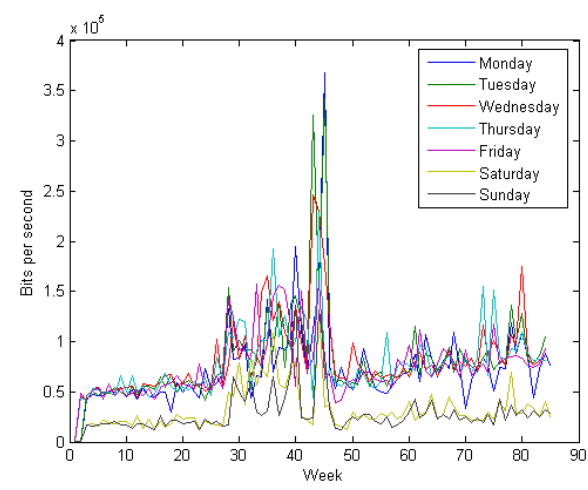

(b) Data Set \#1

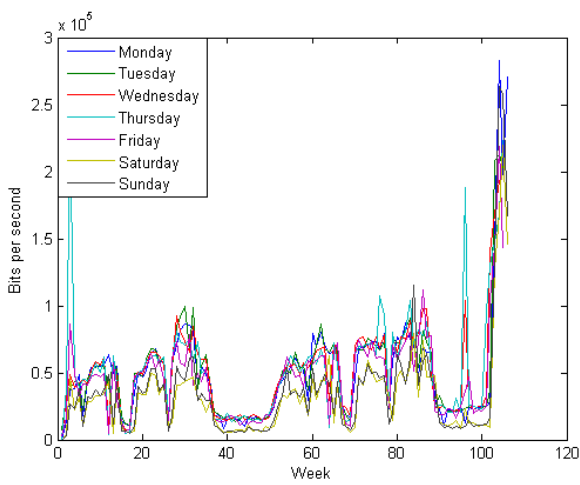

(d) Data Set \#3

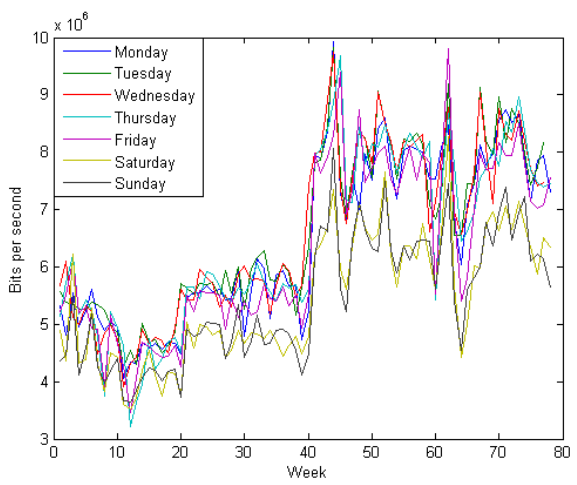

(f) Data Set \#5

Figure 2.3: The inbound traffic by the day of the week. There appears to be a seasonal component on the weekends to these data sets. A significance test was performed to verify this hypothesis. 


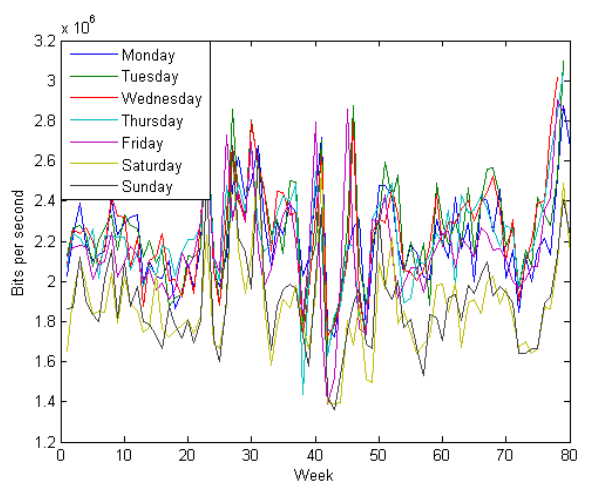

(a) Data Set \#0

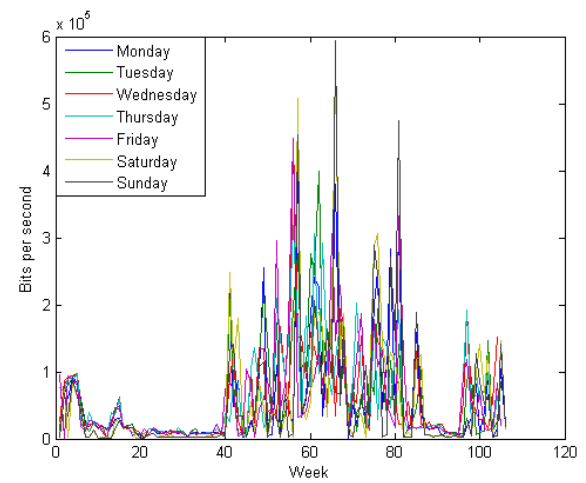

(c) Data Set \#2

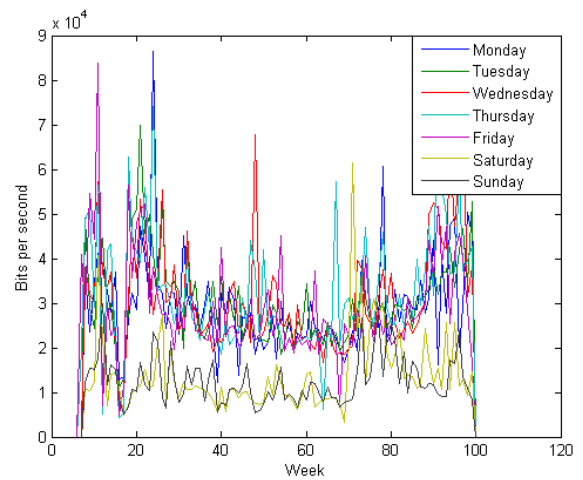

(e) Data Set \#4

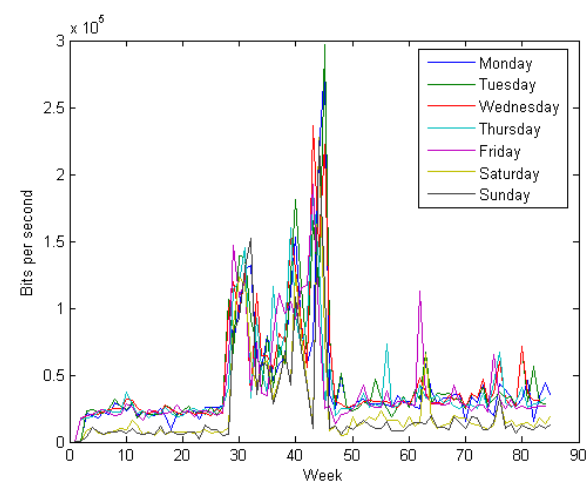

(b) Data Set \#1

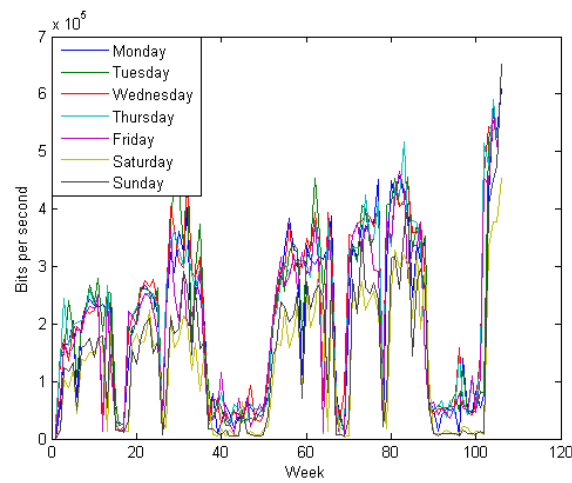

(d) Data Set \#3

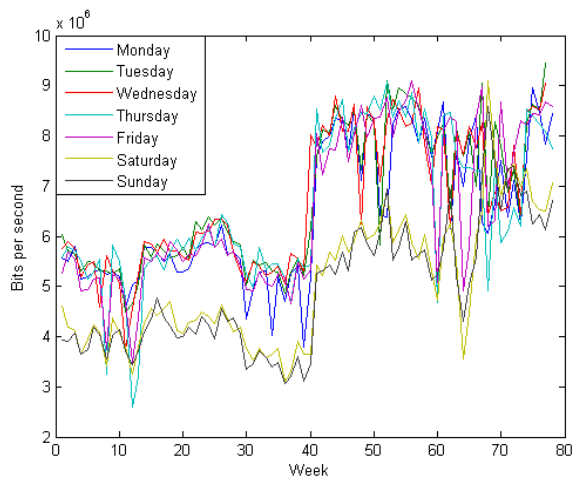

(f) Data Set \#5

Figure 2.4: The outbound traffic by the day of the week. There appears to be a seasonal component on the weekends to these data sets. A significance test was performed to verify this hypothesis. 
weekends to the majority of these data sets. The outbound traffic for data set \#2 is the only data set that does not have a seasonal component.

\subsubsection{Test for long-range dependence}

Hill's estimator was used to determine the value of $\alpha$, which represents the level of long-range dependence. Figures 2.5 and 2.6 shows the Hill's plot for the inbound and outbound traffic for all six data sets. Table 2.8 shows the estimated $\alpha$ parameter for all six data sets.

There are times when the Hill plot will not stabilize. In cases like this, the Hill plot will need to be computed multiple times until it stabilizes. Figure 2.7 shows the computed Hill Plot for the inbound traffic on data set \#4.

Table 2.8 also shows the value of $\alpha$ using the $\alpha=2-2 H$ formula, where $\mathrm{H}$ is the Hurst parameter. There is a discrepancy in the value of $\alpha$ between Hill's estimator and by using this formula.

\subsubsection{Signal decomposition using STL}

Recall that the classical decomposition model [1] is represented by the following equation:

$$
X_{t}=m_{t}+s_{t}+Y_{t}
$$

where $m_{t}$ is the trend component, $s_{t}$ is the seasonal component and $Y_{t}$ is the random noise component. Figures 2.8 and 2.9 shows the decomposition of the six data sets using the STL algorithm [4]. 


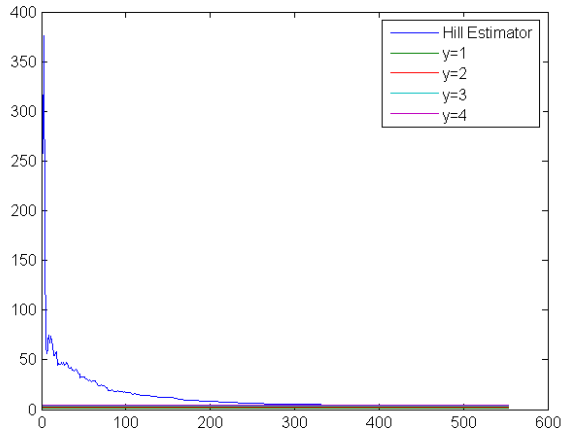

(a) Data Set \#0

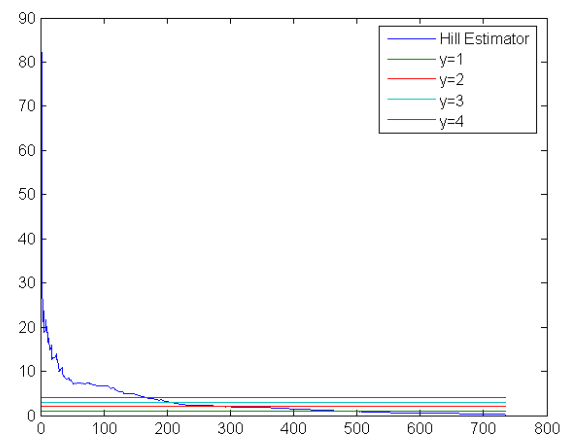

(c) Data Set \#2

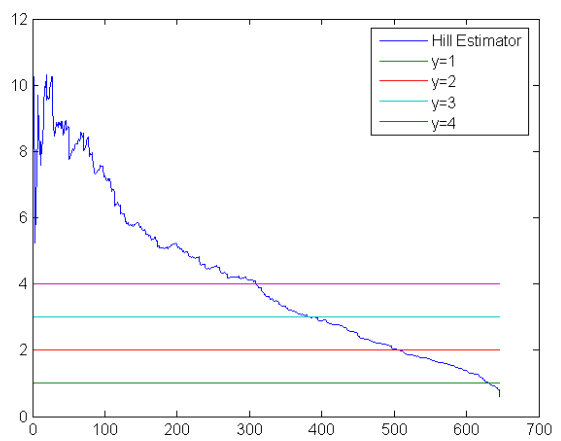

(e) Data Set \#4

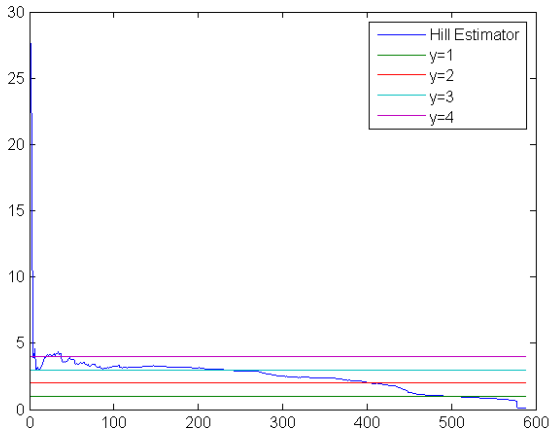

(b) Data Set \#1

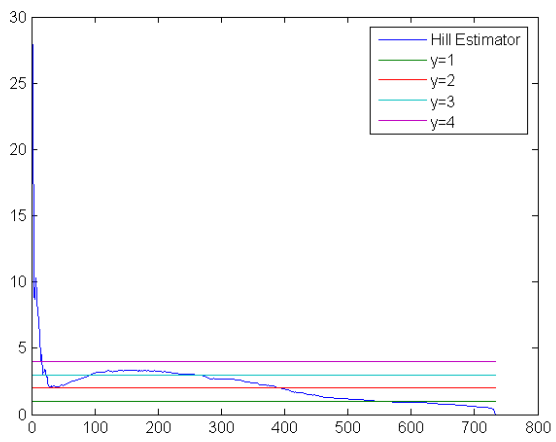

(d) Data Set \#3

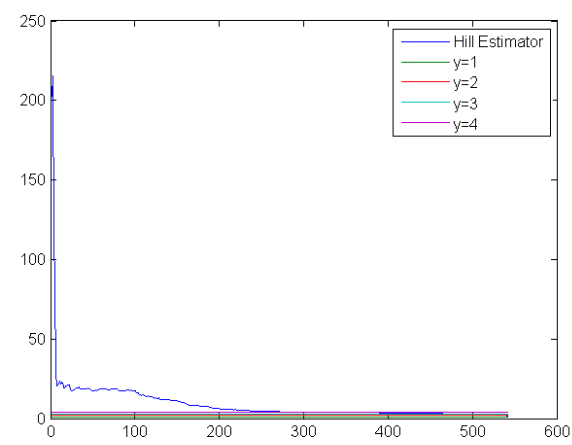

(f) Data Set \#5

Figure 2.5: Hill's estimator on the inbound traffic. 


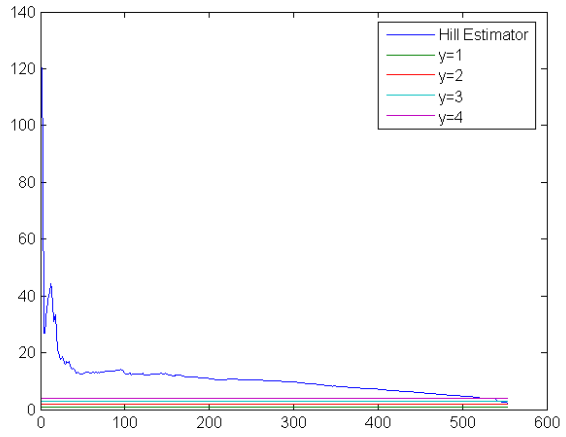

(a) Data Set \#0

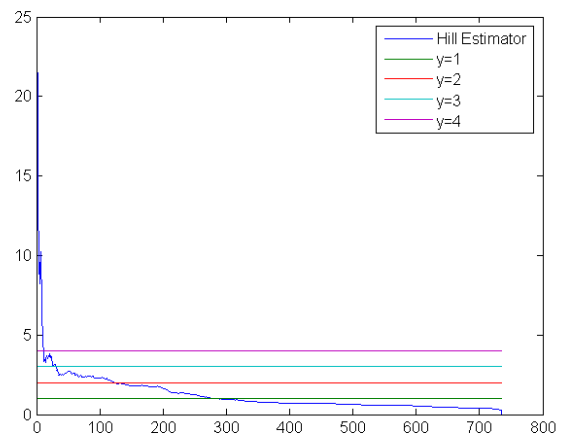

(c) Data Set \#2

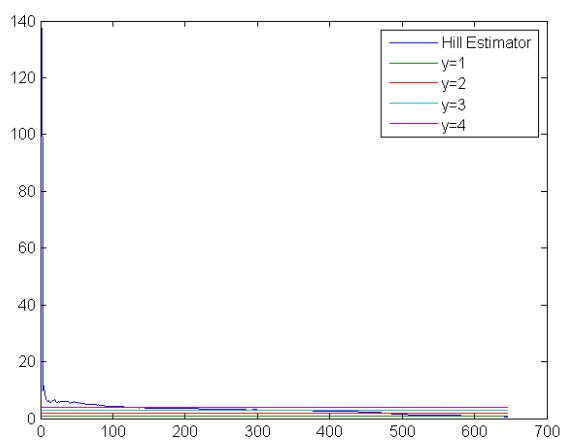

(e) Data Set \#4

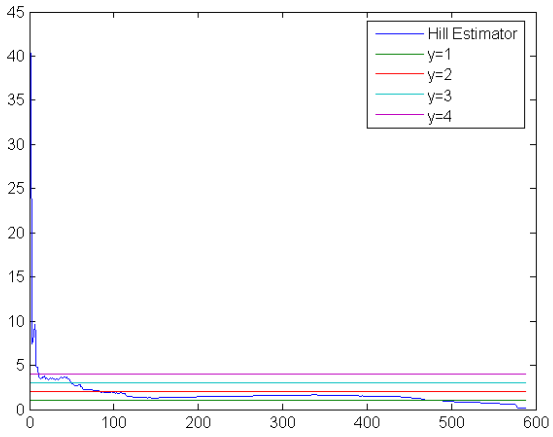

(b) Data Set \#1

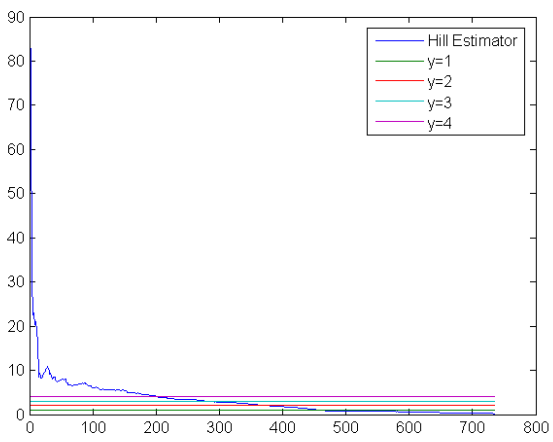

(d) Data Set \#3

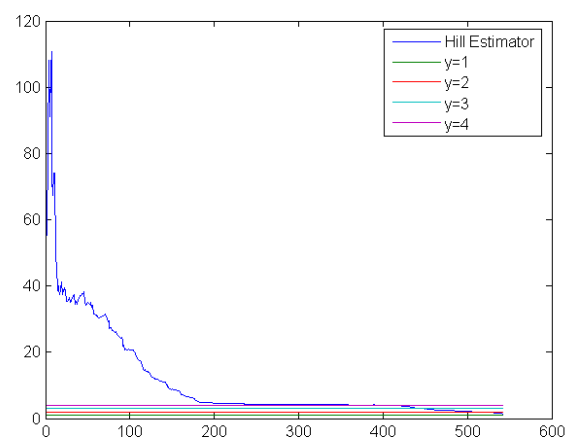

(f) Data Set \#5

Figure 2.6: Hill's estimator on the outbound traffic. 


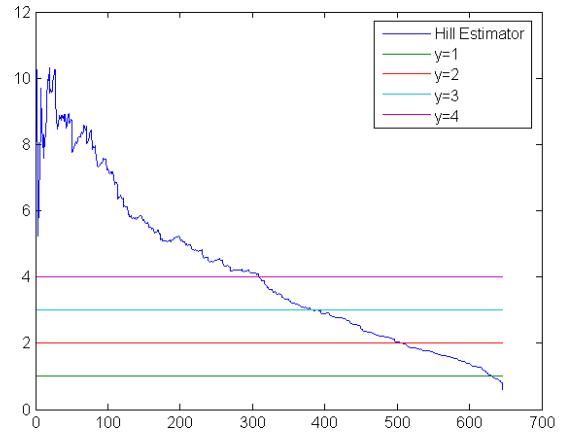

(a) Pass \#1

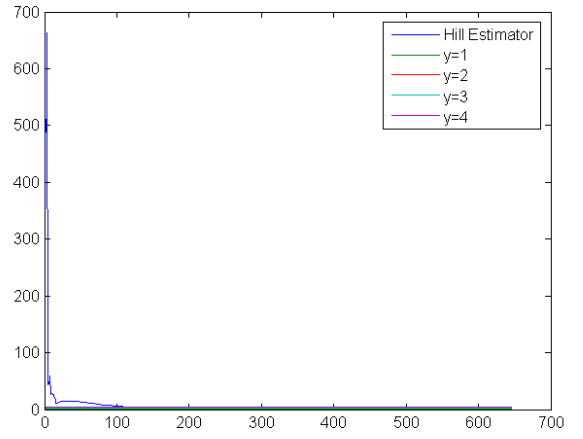

(b) Pass \#2

Figure 2.7: There are times when the Hill plot will not stabilize. In cases like this, the Hill plot will need to be computed multiple times until it stabilizes. These two graphs show the computed Hill Plot for the inbound traffic on data set \#4. 


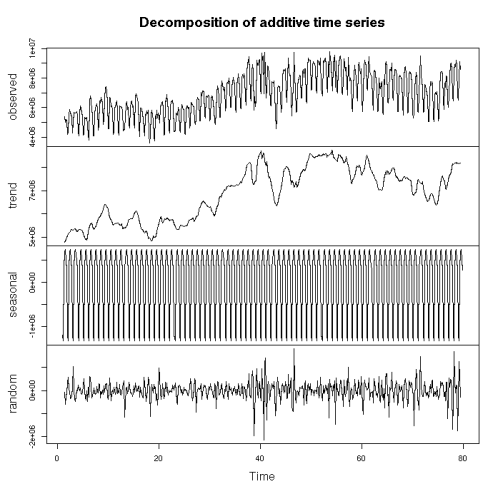

(a) Data Set \#0

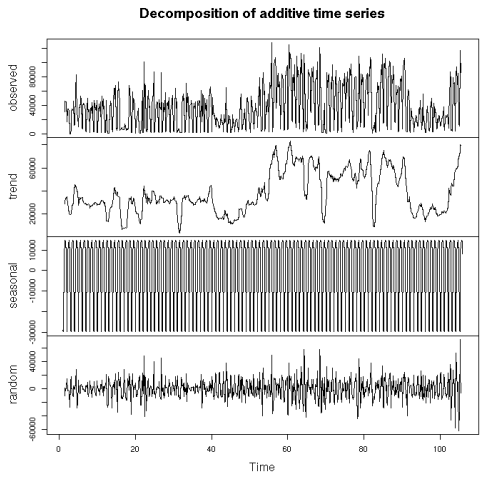

(c) Data Set \#2

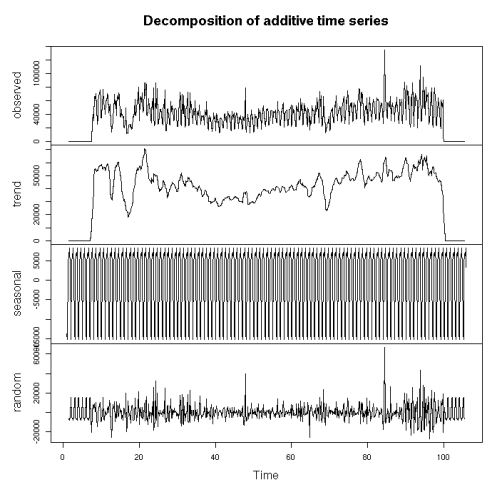

(e) Data Set \#4

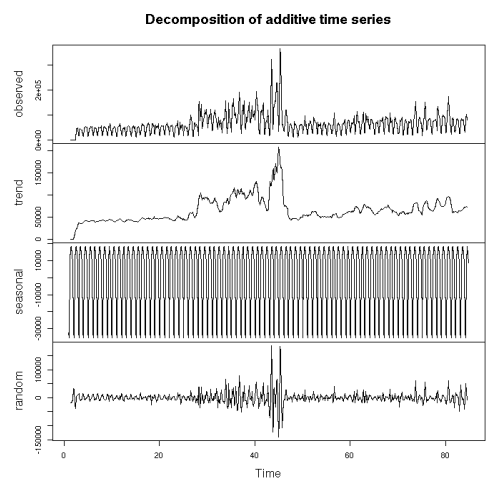

(b) Data Set \#1

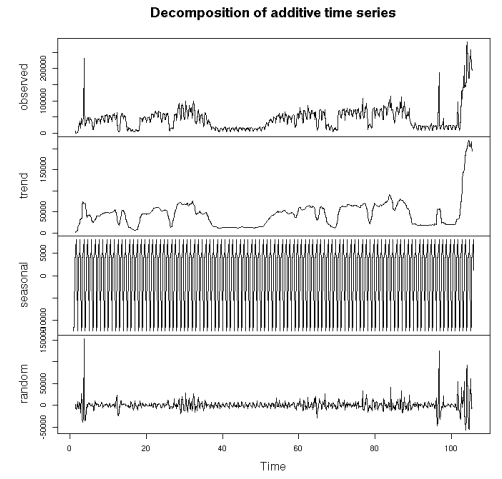

(d) Data Set \#3

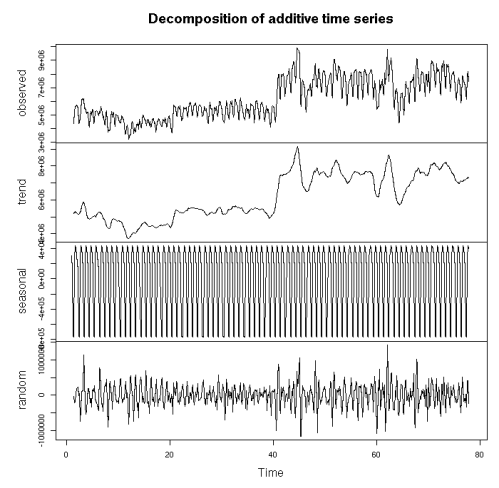

(f) Data Set \#5

Figure 2.8: The classical decomposition of the inbound traffic in the six data sets using the STL algorithm [4]. Recall that the classical decomposition of a time series can be represented as the sum of three components: i) the trend component, ii) the seasonal component and iii) the random noise component. 


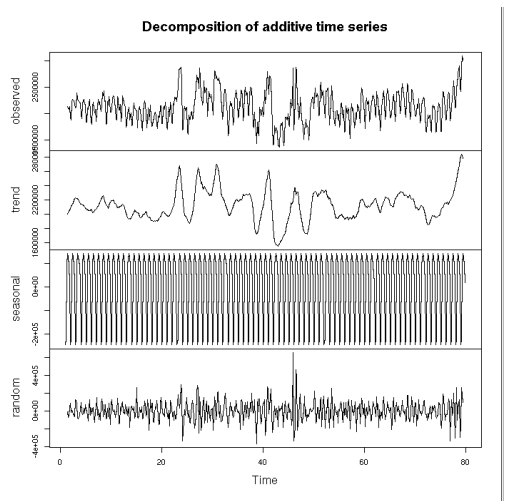

(a) Data Set \#0

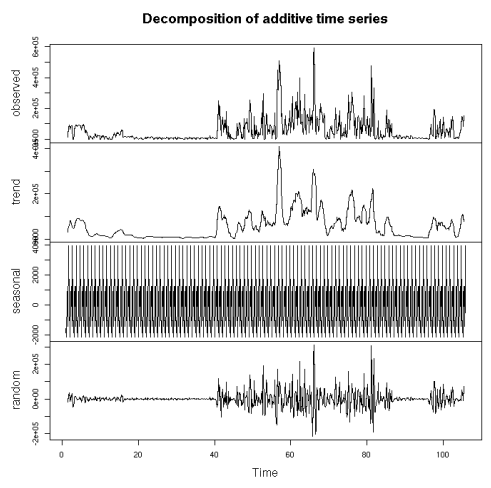

(c) Data Set \#2

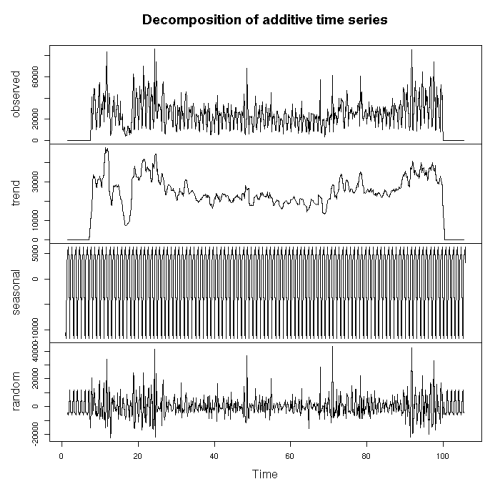

(e) Data Set \#4

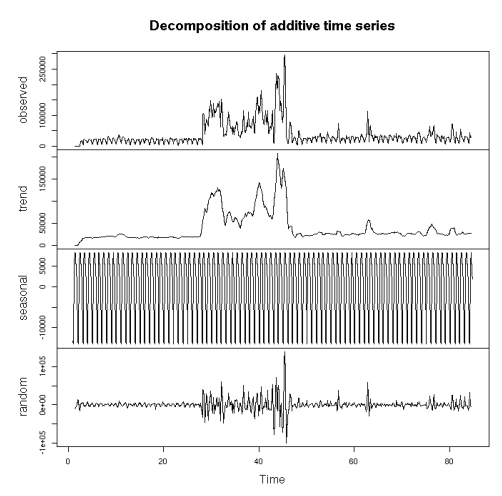

(b) Data Set \#1

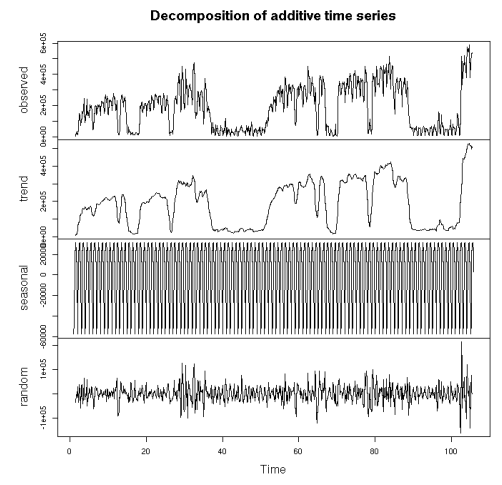

(d) Data Set \#3

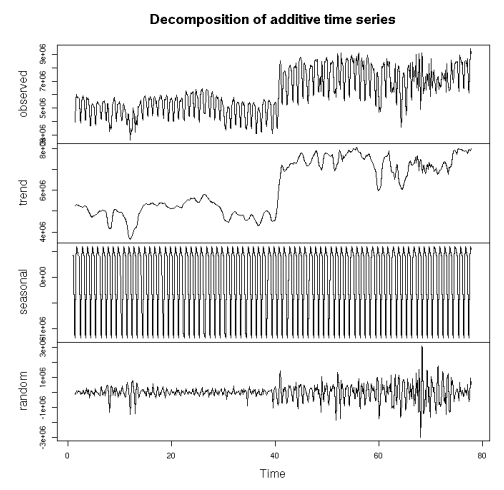

(f) Data Set \#5

Figure 2.9: The classical decomposition of the outbound traffic in the six data sets using the STL algorithm [4]. Recall that the classical decomposition of a time series can be represented as the sum of three components: i) the trend component, ii) the seasonal component and iii) the random noise component. 


\subsubsection{Summary}

There were several properties that were analyzed in all six data sets. The auto-correlation function and the Hurst parameter were calculated for all six data sets. This verified that self similarity was present in all of the sets.

It was noted that the auto-correlation had large fluctuations in the graph. The data sets were split into their corresponding day of the week. T-tests were performed between each day of the week to see if the differences in the daily values are significant. It was found that a seasonal component exists in all but one data set. Specifically the weekends represent the seasonal component.

Hill's estimator was used to determine the value of $\alpha$, which represents the level of long-range dependence. It showed that only a few of the data sets exhibited long-range dependence. Conflicting results were found though when the value of $\alpha$ was generated using the equation $2-2 H$. 


\begin{tabular}{|c|c|c|c|c|c|}
\hline Set \# & Day & Inbound Mean & Inbound Variance & Outbound Mean & Outbound Variance \\
\hline 0 & Mon & 7188447.30 & 1643465630984.54 & 2216703.16 & 54782265706.33 \\
\hline 0 & Tue & 7548798.36 & 1590865724196.60 & 2260490.31 & 70548899212.42 \\
\hline 0 & Wed & 7596471.63 & 1626736966802.21 & 2244439.46 & 69242238551.36 \\
\hline 0 & Thu & 7400188.08 & 1599059811365.14 & 2219335.61 & 63617386093.62 \\
\hline 0 & Fri & 7244688.20 & 1710680952762.55 & 2160806.12 & 70173733335.33 \\
\hline 0 & Sat & 5671381.20 & 1259986411410.39 & 1874029.47 & 58288948479.50 \\
\hline 0 & Sun & 5506824.96 & 1005122259160.38 & 1896912.04 & 58774917290.55 \\
\hline 1 & Mon & 73515.14 & 1937239480.23 & 43783.38 & 1842452178.12 \\
\hline 1 & Tue & 82410.37 & 2433491515.21 & 46841.02 & 1995664828.92 \\
\hline 1 & Wed & 82631.43 & 1512461841.61 & 46632.82 & 1815919665.75 \\
\hline 1 & Thu & 78085.98 & 1137102461.18 & 41830.37 & 1225483136.26 \\
\hline 1 & Fri & 75347.10 & 923686351.52 & 40912.38 & 1218584763.33 \\
\hline 1 & Sat & 32251.19 & 483893581.64 & 25305.85 & 1213975620.17 \\
\hline 1 & Sun & 28504.94 & 465116218.59 & 24081.71 & 1348827686.82 \\
\hline 2 & Mon & 46392.92 & 829066177.46 & 57934.63 & 7081608136.99 \\
\hline 2 & Tue & 51732.27 & 752244542.78 & 58792.81 & 5235403586.83 \\
\hline 2 & Wed & 52386.85 & 748872549.09 & 56901.06 & 4303217233.57 \\
\hline 2 & Thu & 53106.07 & 855388818.71 & 62480.54 & 4893802895.48 \\
\hline 2 & Fri & 48801.44 & 830800769.59 & 57251.72 & 5701603020.08 \\
\hline 2 & Sat & 8819.70 & 63717733.43 & 56271.44 & 8715195951.52 \\
\hline 2 & Sun & 7220.77 & 51188396.05 & 55649.13 & 9940141754.20 \\
\hline 3 & Mon & 50769.04 & 1863367967.18 & 207111.69 & 22486328073.36 \\
\hline 3 & Tue & 50636.38 & 1302886160.90 & 217101.71 & 22446597552.21 \\
\hline 3 & Wed & 50338.97 & 1032126999.83 & 215291.86 & 21386474139.94 \\
\hline 3 & Thu & 53743.82 & 1693261413.11 & 211591.32 & 21457320754.70 \\
\hline 3 & Fri & 46307.25 & 1049027252.25 & 195618.62 & 19316578487.45 \\
\hline 3 & Sat & 32340.36 & 1053888941.60 & 128415.39 & 12728253676.54 \\
\hline 3 & Sun & 35403.57 & 1671505235.24 & 142253.39 & 17359316097.42 \\
\hline 4 & Mon & 42232.37 & 425772981.22 & 25545.32 & 199777439.84 \\
\hline 4 & Tue & 45346.42 & 478769384.08 & 26789.69 & 184910838.22 \\
\hline 4 & Wed & 45226.51 & 425909333.39 & 27867.91 & 237897136.95 \\
\hline 4 & Thu & 46808.84 & 561010983.13 & 28693.20 & 276018753.93 \\
\hline 4 & Fri & 41986.20 & 426037802.34 & 25717.77 & 209018626.48 \\
\hline 4 & Sat & 24745.57 & 188732631.26 & 12132.18 & 86426426.61 \\
\hline 4 & Sun & 23315.60 & 141082210.57 & 10958.19 & 43092387.65 \\
\hline 5 & Mon & 6482325.93 & 2149847840714.85 & 6468380.46 & 1892516674213.35 \\
\hline 5 & Tue & 6639075.84 & 2130942757747.85 & 6743592.72 & 1932342078372.95 \\
\hline 5 & Wed & 6595360.16 & 2215721712251.99 & 6682297.06 & 1851505071582.00 \\
\hline 5 & Thu & 6489405.41 & 2352587983032.93 & 6546998.80 & 2284912859119.95 \\
\hline 5 & Fri & 6309913.99 & 2204282951276.93 & 6539130.83 & 2363590751498.93 \\
\hline 5 & Sat & 5440134.13 & 1251119213856.83 & 5068846.51 & 1697047689139.75 \\
\hline 5 & Sun & 5412079.07 & 1211115660083.45 & 4946653.88 & 1644963121701.11 \\
\hline
\end{tabular}

Table 2.5: The numeric representation of the six data sets by the day of the week. 


\begin{tabular}{|c|c|c|c|c|c|c|c|c|c|c|c|c|c|c|}
\hline & \multicolumn{10}{|c|}{ Inbound } & \multicolumn{1}{c|}{ Outbound } \\
\hline & Mon & Tue & Wed & Thu & Fri & Sat & Sun & Mon & Tue & Wed & Thu & Fri & Sat & Sun \\
\hline Mon & 1.00 & 0.08 & 0.05 & 0.30 & 0.78 & 0.00 & 0.00 & 1.00 & 0.27 & 0.48 & 0.95 & 0.16 & 0.00 & 0.00 \\
Tue & 0.08 & 1.00 & 0.81 & 0.46 & 0.14 & 0.00 & 0.00 & 0.27 & 1.00 & 0.70 & 0.32 & 0.02 & 0.00 & 0.00 \\
Wed & 0.05 & 0.81 & 1.00 & 0.33 & 0.09 & 0.00 & 0.00 & 0.48 & 0.70 & 1.00 & 0.54 & 0.05 & 0.00 & 0.00 \\
Thu & 0.30 & 0.46 & 0.33 & 1.00 & 0.45 & 0.00 & 0.00 & 0.95 & 0.32 & 0.54 & 1.00 & 0.16 & 0.00 & 0.00 \\
Fri & 0.78 & 0.14 & 0.09 & 0.45 & 1.00 & 0.00 & 0.00 & 0.16 & 0.02 & 0.05 & 0.16 & 1.00 & 0.00 & 0.00 \\
Sat & 0.00 & 0.00 & 0.00 & 0.00 & 0.00 & 1.00 & 0.33 & 0.00 & 0.00 & 0.00 & 0.00 & 0.00 & 1.00 & 0.55 \\
Sun & 0.00 & 0.00 & 0.00 & 0.00 & 0.00 & 0.33 & 1.00 & 0.00 & 0.00 & 0.00 & 0.00 & 0.00 & 0.55 & 1.00 \\
\hline
\end{tabular}

(a) Data Set \#0

\begin{tabular}{|c|c|c|c|c|c|c|c|c|c|c|c|c|c|c|}
\hline & \multicolumn{10}{|c|}{ Inbound } & \multicolumn{1}{c|}{ Outbound } \\
\hline & Mon & Tue & Wed & Thu & Fri & Sat & Sun & Mon & Tue & Wed & Thu & Fri & Sat & Sun \\
\hline Mon & 1.00 & 0.22 & 0.16 & 0.45 & 0.75 & 0.00 & 0.00 & 1.00 & 0.65 & 0.67 & 0.75 & 0.63 & 0.00 & 0.00 \\
Tue & 0.22 & 1.00 & 0.97 & 0.51 & 0.27 & 0.00 & 0.00 & 0.65 & 1.00 & 0.98 & 0.42 & 0.34 & 0.00 & 0.00 \\
Wed & 0.16 & 0.97 & 1.00 & 0.42 & 0.18 & 0.00 & 0.00 & 0.67 & 0.98 & 1.00 & 0.43 & 0.34 & 0.00 & 0.00 \\
Thu & 0.45 & 0.51 & 0.42 & 1.00 & 0.58 & 0.00 & 0.00 & 0.75 & 0.42 & 0.43 & 1.00 & 0.87 & 0.00 & 0.00 \\
Fri & 0.75 & 0.27 & 0.18 & 0.58 & 1.00 & 0.00 & 0.00 & 0.63 & 0.34 & 0.34 & 0.87 & 1.00 & 0.00 & 0.00 \\
Sat & 0.00 & 0.00 & 0.00 & 0.00 & 0.00 & 1.00 & 0.26 & 0.00 & 0.00 & 0.00 & 0.00 & 0.00 & 1.00 & 0.82 \\
Sun & 0.00 & 0.00 & 0.00 & 0.00 & 0.00 & 0.26 & 1.00 & 0.00 & 0.00 & 0.00 & 0.00 & 0.00 & 0.82 & 1.00 \\
\hline
\end{tabular}

(b) Data Set \#1

\begin{tabular}{|c|c|c|c|c|c|c|c|c|c|c|c|c|c|c|}
\hline & \multicolumn{10}{|c|}{ Inbound } \\
\hline & Mon & Tue & Wed & Thu & Fri & Sat & Sun & Mon & Tue & Wed & Thu & Fri & Sat & Sun \\
\hline Mon & 1.00 & 0.17 & 0.12 & 0.09 & 0.54 & 0.00 & 0.00 & 1.00 & 0.94 & 0.92 & 0.67 & 0.95 & 0.89 & 0.86 \\
Tue & 0.17 & 1.00 & 0.86 & 0.73 & 0.45 & 0.00 & 0.00 & 0.94 & 1.00 & 0.84 & 0.71 & 0.88 & 0.83 & 0.79 \\
Wed & 0.12 & 0.86 & 1.00 & 0.85 & 0.36 & 0.00 & 0.00 & 0.92 & 0.84 & 1.00 & 0.55 & 0.97 & 0.96 & 0.91 \\
Thu & 0.09 & 0.73 & 0.85 & 1.00 & 0.28 & 0.00 & 0.00 & 0.67 & 0.71 & 0.55 & 1.00 & 0.60 & 0.59 & 0.57 \\
Fri & 0.54 & 0.45 & 0.36 & 0.28 & 1.00 & 0.00 & 0.00 & 0.95 & 0.88 & 0.97 & 0.60 & 1.00 & 0.93 & 0.90 \\
Sat & 0.00 & 0.00 & 0.00 & 0.00 & 0.00 & 1.00 & 0.13 & 0.89 & 0.83 & 0.96 & 0.59 & 0.93 & 1.00 & 0.96 \\
Sun & 0.00 & 0.00 & 0.00 & 0.00 & 0.00 & 0.13 & 1.00 & 0.86 & 0.79 & 0.91 & 0.57 & 0.90 & 0.96 & 1.00 \\
\hline
\end{tabular}

(c) Data Set \#2

Table 2.6: The p-values from the t-tests that were performed on data sets \#0-2 to determine the significance of the mean and variance between each day of the week. This data shows that there is a seasonal component on the weekends to the majority of these data sets. The outbound traffic for data set \#2 is the only data set that does not have a seasonal component. 


\begin{tabular}{|c|c|c|c|c|c|c|c|c|c|c|c|c|c|c|}
\hline & \multicolumn{10}{|c|}{ Inbound } & \multicolumn{1}{c|}{ Outbound } \\
\hline & Mon & Tue & Wed & Thu & Fri & Sat & Sun & Mon & Tue & Wed & Thu & Fri & Sat & Sun \\
\hline Mon & 1.00 & 0.98 & 0.93 & 0.61 & 0.40 & 0.00 & 0.01 & 1.00 & 0.63 & 0.69 & 0.83 & 0.56 & 0.00 & 0.00 \\
Tue & 0.98 & 1.00 & 0.95 & 0.56 & 0.36 & 0.00 & 0.00 & 0.63 & 1.00 & 0.93 & 0.79 & 0.28 & 0.00 & 0.00 \\
Wed & 0.93 & 0.95 & 1.00 & 0.51 & 0.37 & 0.00 & 0.00 & 0.69 & 0.93 & 1.00 & 0.86 & 0.32 & 0.00 & 0.00 \\
Thu & 0.61 & 0.56 & 0.51 & 1.00 & 0.15 & 0.00 & 0.00 & 0.83 & 0.79 & 0.86 & 1.00 & 0.42 & 0.00 & 0.00 \\
Fri & 0.40 & 0.36 & 0.37 & 0.15 & 1.00 & 0.00 & 0.03 & 0.56 & 0.28 & 0.32 & 0.42 & 1.00 & 0.00 & 0.00 \\
Sat & 0.00 & 0.00 & 0.00 & 0.00 & 0.00 & 1.00 & 0.55 & 0.00 & 0.00 & 0.00 & 0.00 & 0.00 & 1.00 & 0.41 \\
Sun & 0.01 & 0.00 & 0.00 & 0.00 & 0.03 & 0.55 & 1.00 & 0.00 & 0.00 & 0.00 & 0.00 & 0.00 & 0.41 & 1.00 \\
\hline
\end{tabular}

(a) Data Set \#3

\begin{tabular}{|c|c|c|c|c|c|c|c|c|c|c|c|c|c|c|}
\hline & \multicolumn{10}{|c|}{ Inbound } & \multicolumn{1}{c|}{ Outbound } \\
\hline & Mon & Tue & Wed & Thu & Fri & Sat & Sun & Mon & Tue & Wed & Thu & Fri & Sat & Sun \\
\hline Mon & 1.00 & 0.29 & 0.29 & 0.14 & 0.93 & 0.00 & 0.00 & 1.00 & 0.52 & 0.26 & 0.14 & 0.93 & 0.00 & 0.00 \\
Tue & 0.29 & 1.00 & 0.97 & 0.64 & 0.25 & 0.00 & 0.00 & 0.52 & 1.00 & 0.59 & 0.36 & 0.58 & 0.00 & 0.00 \\
Wed & 0.29 & 0.97 & 1.00 & 0.61 & 0.26 & 0.00 & 0.00 & 0.26 & 0.59 & 1.00 & 0.71 & 0.30 & 0.00 & 0.00 \\
Thu & 0.14 & 0.64 & 0.61 & 1.00 & 0.12 & 0.00 & 0.00 & 0.14 & 0.36 & 0.71 & 1.00 & 0.17 & 0.00 & 0.00 \\
Fri & 0.93 & 0.25 & 0.26 & 0.12 & 1.00 & 0.00 & 0.00 & 0.93 & 0.58 & 0.30 & 0.17 & 1.00 & 0.00 & 0.00 \\
Sat & 0.00 & 0.00 & 0.00 & 0.00 & 0.00 & 1.00 & 0.42 & 0.00 & 0.00 & 0.00 & 0.00 & 0.00 & 1.00 & 0.29 \\
Sun & 0.00 & 0.00 & 0.00 & 0.00 & 0.00 & 0.42 & 1.00 & 0.00 & 0.00 & 0.00 & 0.00 & 0.00 & 0.29 & 1.00 \\
\hline
\end{tabular}

(b) Data Set \#4

\begin{tabular}{|c|c|c|c|c|c|c|c|c|c|c|c|c|c|c|}
\hline & \multicolumn{10}{|c|}{ Inbound } \\
\hline & Mon & Tue & Wed & Thu & Fri & Sat & Sun & Mon & Tue & Wed & Thu & Fri & Sat & Sun \\
\hline Mon & 1.00 & 0.51 & 0.63 & 0.98 & 0.47 & 0.00 & 0.00 & 1.00 & 0.22 & 0.33 & 0.73 & 0.76 & 0.00 & 0.00 \\
Tue & 0.51 & 1.00 & 0.85 & 0.53 & 0.17 & 0.00 & 0.00 & 0.22 & 1.00 & 0.78 & 0.40 & 0.39 & 0.00 & 0.00 \\
Wed & 0.63 & 0.85 & 1.00 & 0.66 & 0.23 & 0.00 & 0.00 & 0.33 & 0.78 & 1.00 & 0.56 & 0.54 & 0.00 & 0.00 \\
Thu & 0.98 & 0.53 & 0.66 & 1.00 & 0.46 & 0.00 & 0.00 & 0.73 & 0.40 & 0.56 & 1.00 & 0.97 & 0.00 & 0.00 \\
Fri & 0.47 & 0.17 & 0.23 & 0.46 & 1.00 & 0.00 & 0.00 & 0.76 & 0.39 & 0.54 & 0.97 & 1.00 & 0.00 & 0.00 \\
Sat & 0.00 & 0.00 & 0.00 & 0.00 & 0.00 & 1.00 & 0.87 & 0.00 & 0.00 & 0.00 & 0.00 & 0.00 & 1.00 & 0.56 \\
Sun & 0.00 & 0.00 & 0.00 & 0.00 & 0.00 & 0.87 & 1.00 & 0.00 & 0.00 & 0.00 & 0.00 & 0.00 & 0.56 & 1.00 \\
\hline
\end{tabular}

(c) Data Set \#5

Table 2.7: The p-values from the t-tests that were performed on data sets \#3-5 to determine the significance of the mean and variance between each day of the week. The analysis show there is a weekend seasonal component within all these data sets. 


\begin{tabular}{|c||c|c||c|c|}
\hline \multirow{2}{*}{ Data Set \# } & \multicolumn{2}{c||}{ Hill's Estimator } & \multicolumn{2}{c|}{$\alpha=2-2 H$} \\
& Inbound $\alpha$ & Outbound $\alpha$ & Inbound $\alpha$ & Outbound $\alpha$ \\
\hline 0 & 5.5 & 6.2 & 0.34514 & 0.73160 \\
1 & 3.2 & 1.3 & 0.50818 & 0.37752 \\
2 & 2.2 & 1.7 & 0.47532 & 0.42914 \\
3 & 1.1 & 3.3 & 0.59000 & 0.46262 \\
4 & 3.1 (two passes) & 3.5 & 0.45724 & 0.51344 \\
5 & 4.1 & 4.2 & 0.28144 & 0.31120 \\
\hline
\end{tabular}

Table 2.8: The estimated $\alpha$ parameter for the six data sets. Recall that $\alpha$ values less than 1 represent data sets that exhibit long-range dependence. 


\section{METHODOLOGY}

This chapter describes the models that were built for each of the data sets. Each data set that was analyzed was split into two distinct sets: a training set and a validation set. The training set was used to build the model and the validation set was used to test the validity of that model.

The data sets were smoothed using Principal Component Analysis (PCA) and Independent Component Analysis (ICA) in an attempt to isolate the overall long-term trend in the data sets. The isolated signal was used to build a model that can be used to predict the future traffic utilization of each of the data sets.

The data sets that were analyzed had a seasonal component on the weekends. Due to the weekly seasonal component, the data was setup in a $\mathrm{N}$ by 7 matrix, where each column represents the day of the week and each of the $\mathrm{N}$ rows represents the corresponding week. Each row of the matrix represents a sample and the columns are the features of the samples. The PCA and ICA algorithms needed the data to be separated into these two categories so that the underlying components can be isolated. 


\subsection{Smoothing using Principle Component Analysis (PCA)}

The eigenvalues and associated eigenvectors were computed for each of the data sets. Since each sample has seven features, there will be seven eigenvectors and eigenvalues. The eigenvectors were sorted in decreasing order by their eigenvalue. The top $\mathrm{k}$ eigenvectors whose eigenvalues represent the top $95 \%$ of the total variance were kept and placed in the matrix A, which will have the dimensions $\mathrm{k}$ by $\mathrm{N}$. The data was transformed into a new coordinate system by using the following equation:

$$
Y=A^{t}(X-\mu)
$$

where $\mathrm{A}$ is a matrix that contains the reduced number of eigenvectors, $\mathrm{X}$ is the original signal and $\mu$ is the mean of $\mathrm{X}$. $\mathrm{Y}$ will have the dimensions $\mathrm{N}$ by $\mathrm{R}$, where $\mathrm{N}$ is the number of samples (or weeks) in the data set and $\mathrm{R}$ is the reduced number of eigenvectors. The reduced data set can be transformed into the original coordinate system by using the following equation:

$$
X^{\prime}=Y \cdot E+\mu
$$

where $\mathrm{Y}$ is the projected data, $\mathrm{E}$ is the transformation (or rotation) matrix and $\mu$ is the mean of the original data set $(\mathrm{X})$.

The training data was used to build an ARIMA model of order $(\mathrm{p}, \mathrm{d}, \mathrm{q})$ where $\mathrm{p}<12$, $\mathrm{q}$ $<12$ and d was typically 1 . An ARIMA model was built for each value of $(\mathrm{p}, \mathrm{d}, \mathrm{q})$ and the

results were tested on the validation set. The model that gave suitable results that had the 
lowest order of $(p, d, q)$ was chosen. This model was used to predict the future values in the time series.

\subsection{Smoothing using Independent Component Analysis (ICA)}

The data was smoothed using PCA as described in the previous section. This produced a matrix $\mathrm{X}^{\prime}$ which has the dimensions $\mathrm{N}$ by 7 , where each column represents the day of the week and each of the $\mathrm{N}$ rows is the corresponding week.

The mixing matrix (A), inverse mixing matrix (W) and the estimate of the source matrix (S) was calculated for the smoothed data set. There are a maximum of seven independent components that are recovered from the data set. It is desirable to find the component that corresponds to the overall long-term trend in an automated fashion. The ICA algorithm does not return the list of components in the same order between successive runs. The correlation between each independent component and the original data set is compared. It was found that only one component will have a high correlation $(>80)$ with the original data set while the other components will have a low correlation. The component that corresponds to the long-term trend is used to modify the source matrix in the following manner:

$$
S^{\prime}=S(t)
$$

where $t$ is the index of the overall long term trend. The matrix $S$ ' will have the dimensions $\mathrm{N}$ by 1 . A similar modification needs to be made to the mixing matrix $(\mathrm{A})$ : 


$$
A^{\prime}=A(t)
$$

where $t$ is the index of the overall long term trend. The matrix A' will have the dimensions 1 by 7 . The smoothed data can now be recovered using the following equation:

$$
Z^{\prime}=S^{\prime} \cdot A^{\prime} .
$$

This will form a $\mathrm{N}$ by 7 matrix that has the data that was smoothed using PCA and ICA.

\subsection{Anomaly detection}

The characteristics of the network traffic may change over time. As these changes occur, the model that was built will no longer be an accurate representation of the data. There comes a time when the model should be regenerated. Figure 3.1 shows an example of some anomalies that may be present in the traffic utilization.

Recall that there may be short and long-term fluctuations in the traffic utilization. The short-term fluctuations may last from a few minutes to a few days. The model should not be rebuilt for the short-term fluctuations since it will cause more errors in the future after these fluctuations have passed.

It is recommended to look at the overall prediction error for the last one or two weeks. The model should only be rebuilt if one of the following conditions are true: i) if the overall prediction error for that period of time is above a certain threshold or ii) if the correlation between the predicted model and the observed values fall below a certain threshold. 


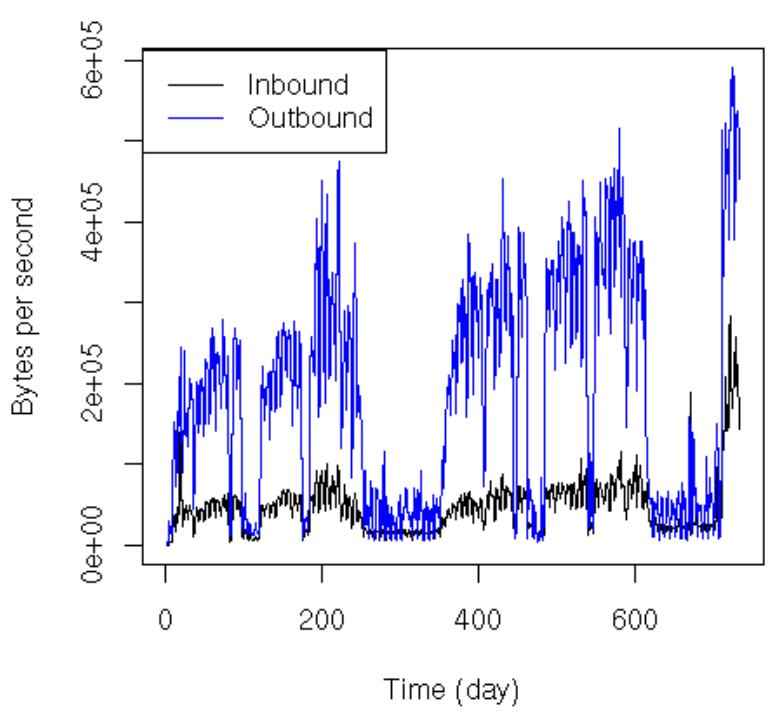

Figure 3.1: A example of some anomalies that may be present in the traffic utilization. 


\section{RESULTS}

There were three forecasting algorithms that were used on the data sets. The first method used an ARIMA model to predict the traffic utilization six months into the future. The second method used PCA to smooth the data. An ARIMA model of this smoothed data was built to predict the future traffic. The third method used PCA and ICA to smooth the data. An ARIMA model of this smoothed data was built to predict the future traffic.

\subsection{Prediction error}

There needs to be a way numerically quantify the results that are achieved by any prediction scheme. This is so that the results from two separate prediction schemes can be compared with each other. This thesis will use two formulas to measure the results from any given prediction algorithm.

The first measure of error is the root Mean Square Error (MSE). It is defined by the following equation:

$$
\frac{\sum_{i=1}^{N} \sqrt{\left(x_{i}-x_{i}^{\prime}\right)^{2}}}{N}
$$

where $x_{i}$ is the $i$ th observed sample from the data set, $x_{i}^{\prime}$ is the $i$ th predicted sample and $\mathrm{N}$ 
is the total number of samples in the data set.

The next measure of error that is employed is the percentage error. It is defined by the following equation:

$$
\frac{\sum_{i=1}^{N} \sqrt{\left(x_{i}-x_{i}^{\prime}\right)^{2}}}{\sum_{i=1}^{N} x_{i}}
$$

where $x_{i}$ is the $i$ th observed sample from the data set, $x_{i}^{\prime}$ is the $i$ th predicted sample and N is the total number of samples in the data set.

\subsection{Traffic prediction using ARIMA}

An ARIMA model was generated for each of the six data sets. These models were used to predict the traffic utilization six months into the future. Table 4.1 shows a summary of the numeric results that were obtained. Figures 4.1 and 4.2 shows a graphical representation of the results that were obtained.

\begin{tabular}{|c|c|c|c|c|}
\hline Data Set \# & ARIMA Parameters & Root MSE (bytes) & Daily \% Error & Weekly \% Error \\
\hline \multirow{2}{*}{0} & $(8,1,7)$ & $149,687.55$ & $26.24 \%$ & $2.13 \%$ \\
& $(6,1,8)$ & $30,419.48$ & $18.99 \%$ & $2.56 \%$ \\
\hline \multirow{2}{*}{1} & $(7,1,8)$ & $4,773.81$ & $64.73 \%$ & $8.32 \%$ \\
& $(4,1,5)$ & $5,010.41$ & $83.55 \%$ & $13.90 \%$ \\
\hline \multirow{2}{*}{2} & $(7,1,7)$ & $3,858.23$ & $77.57 \%$ & $10.04 \%$ \\
& $(4,1,4)$ & $8,268.16$ & $66.38 \%$ & $20.33 \%$ \\
\hline \multirow{2}{*}{3} & $(7,1,7)$ & $2,008.94$ & $49.07 \%$ & $17.01 \%$ \\
& $(10,1,5)$ & $14,041.76$ & $66.59 \%$ & $19.52 \%$ \\
\hline \multirow{2}{*}{4} & $(5,1,7)$ & $1,384.49$ & $45.43 \%$ & $7.15 \%$ \\
& $(5,1,5)$ & $1,168.75$ & $67.80 \%$ & $7.84 \%$ \\
\hline \multirow{2}{*}{5} & $(4,1,6)$ & $193,233.70$ & $39.62 \%$ & $2.56 \%$ \\
& $(8,1,6)$ & $187,415.65$ & $40.36 \%$ & $1.42 \%$ \\
\hline
\end{tabular}

Table 4.1: The estimated ARIMA parameters for the six data sets. The root Mean Square Error (MSE) along with the corresponding prediction error are also shown. 


\subsection{Traffic prediction using PCA and ARIMA}

In an attempt to obtain better results, the data set was smoothed using Principal Component Analysis (PCA). Figures 4.3 and 4.4 shows a graph of the variances for the seven principal components. The numeric representation can be found in Tables 4.2 and 4.3 .

\begin{tabular}{|c|c|c|c|c|c|c|c|c|}
\hline Data Set \# & & PC1 & PC2 & PC3 & PC4 & PC5 & PC6 & PC7 \\
\hline \multirow{2}{*}{0} & Std. Dev. & 2.50 & 0.55 & 0.40 & 0.34 & 0.31 & 0.27 & 0.17 \\
& Var. Prop. & 0.89 & 0.04 & 0.02 & 0.02 & 0.01 & 0.01 & 0.00 \\
\hline \multirow{2}{*}{1} & Std. Dev. & 2.03 & 1.07 & 0.92 & 0.60 & 0.54 & 0.40 & 0.26 \\
& Var. Prop. & 0.59 & 0.16 & 0.12 & 0.05 & 0.04 & 0.02 & 0.01 \\
\hline \multirow{2}{*}{2} & Std. Dev. & 2.04 & 0.97 & 0.87 & 0.68 & 0.57 & 0.46 & 0.37 \\
& Var. Prop. & 0.60 & 0.13 & 0.11 & 0.07 & 0.05 & 0.03 & 0.02 \\
\hline \multirow{2}{*}{3} & Std. Dev. & 2.46 & 0.68 & 0.43 & 0.34 & 0.28 & 0.23 & 0.16 \\
& Var. Prop. & 0.87 & 0.07 & 0.03 & 0.02 & 0.01 & 0.01 & 0.00 \\
\hline \multirow{2}{*}{4} & Std. Dev. & 2.01 & 0.90 & 0.79 & 0.73 & 0.64 & 0.55 & 0.52 \\
& Var. Prop. & 0.58 & 0.12 & 0.09 & 0.08 & 0.06 & 0.04 & 0.04 \\
\hline \multirow{2}{*}{5} & Std. Dev. & 2.39 & 0.79 & 0.58 & 0.36 & 0.32 & 0.25 & 0.17 \\
& Var. Prop. & 0.82 & 0.09 & 0.05 & 0.02 & 0.02 & 0.01 & 0.00 \\
\hline
\end{tabular}

Table 4.2: The standard deviation and the proportion of variance for the 7 principal components in the inbound data sets.

\begin{tabular}{|c|c|c|c|c|c|c|c|c|}
\hline Data Set \# & & PC1 & PC2 & PC3 & PC4 & PC5 & PC6 & PC7 \\
\hline \multirow{2}{*}{0} & Std. Dev. & 2.22 & 1.05 & 0.60 & 0.50 & 0.43 & 0.34 & 0.26 \\
& Var. Prop. & 0.70 & 0.16 & 0.05 & 0.04 & 0.03 & 0.02 & 0.01 \\
\hline \multirow{2}{*}{1} & Std. Dev. & 2.26 & 1.06 & 0.66 & 0.36 & 0.35 & 0.20 & 0.17 \\
& Var. Prop. & 0.73 & 0.16 & 0.06 & 0.02 & 0.02 & 0.01 & 0.00 \\
\hline \multirow{2}{*}{2} & Std. Dev. & 1.99 & 1.15 & 0.77 & 0.72 & 0.58 & 0.48 & 0.23 \\
& Var. Prop. & 0.56 & 0.19 & 0.09 & 0.07 & 0.05 & 0.03 & 0.01 \\
\hline \multirow{2}{*}{3} & Std. Dev. & 2.52 & 0.60 & 0.35 & 0.26 & 0.20 & 0.17 & 0.13 \\
& Var. Prop. & 0.91 & 0.05 & 0.02 & 0.01 & 0.01 & 0.00 & 0.00 \\
\hline \multirow{2}{*}{4} & Std. Dev. & 1.73 & 1.09 & 0.99 & 0.80 & 0.73 & 0.59 & 0.55 \\
& Var. Prop. & 0.43 & 0.17 & 0.14 & 0.09 & 0.08 & 0.05 & 0.04 \\
\hline \multirow{2}{*}{5} & Std. Dev. & 2.54 & 0.55 & 0.30 & 0.25 & 0.20 & 0.14 & 0.11 \\
& Var. Prop. & 0.92 & 0.04 & 0.01 & 0.01 & 0.01 & 0.00 & 0.00 \\
\hline
\end{tabular}

Table 4.3: The standard deviation and the proportion of variance for the 7 principal components in the outbound data sets. 


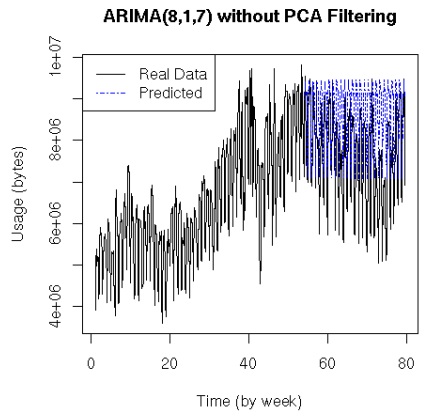

(a) Data Set \#0 Inbound

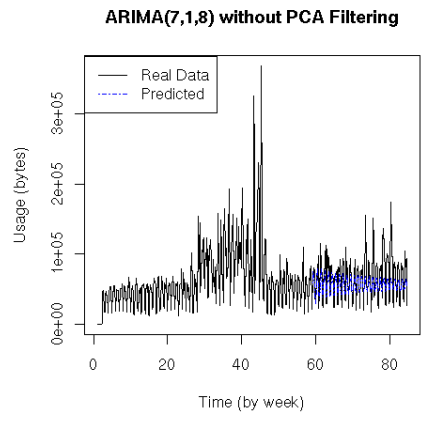

(c) Data Set \#1 Inbound

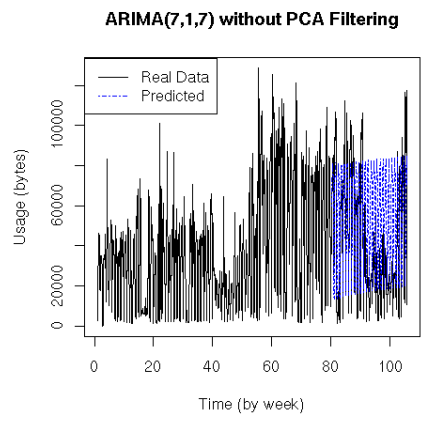

(e) Data Set \#2 Inbound

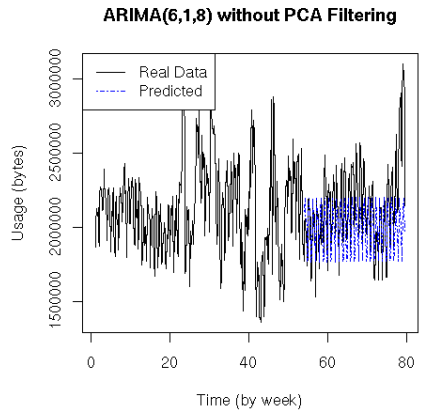

(b) Data Set \#0 Outbound

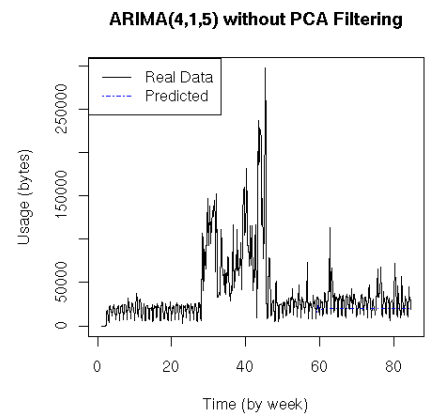

(d) Data Set \#1 Outbound

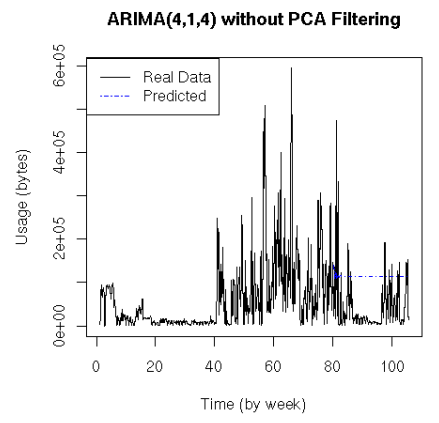

(f) Data Set \#2 Outbound

Figure 4.1: An ARIMA model of the data sets was generated. This model was used to predict the traffic six months into the future. The results are shown for data sets $\# 0-2$. 


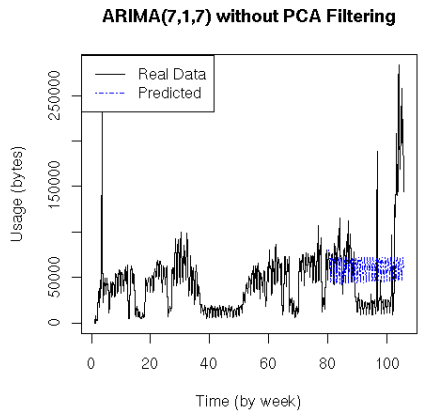

(a) Data Set \#3 Inbound

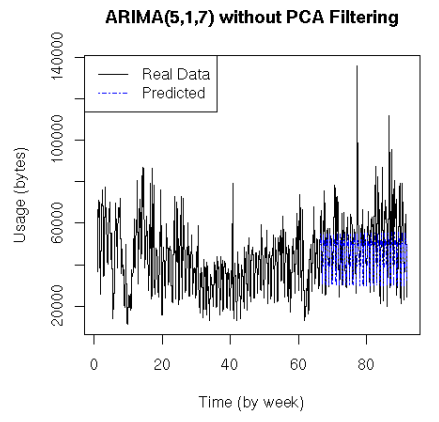

(c) Data Set \#4 Inbound

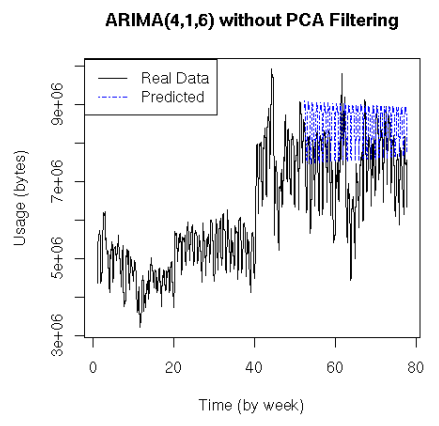

(e) Data Set \#5 Inbound

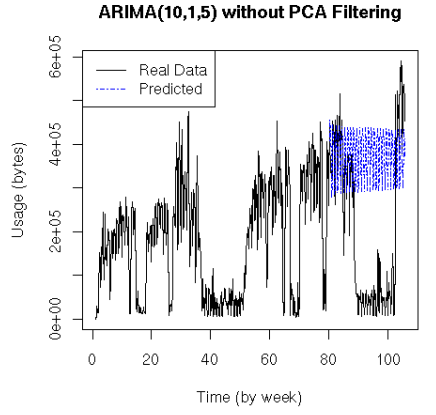

(b) Data Set \#3 Outbound

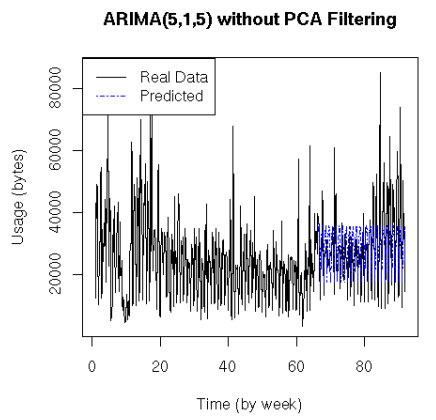

(d) Data Set \#4 Outbound

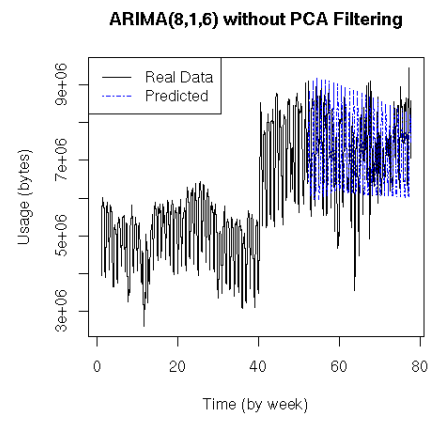

(f) Data Set \#5 Outbound

Figure 4.2: An ARIMA model of the data sets was generated. This model was used to predict the traffic six months into the future. The results are shown for data sets \#3-5. 

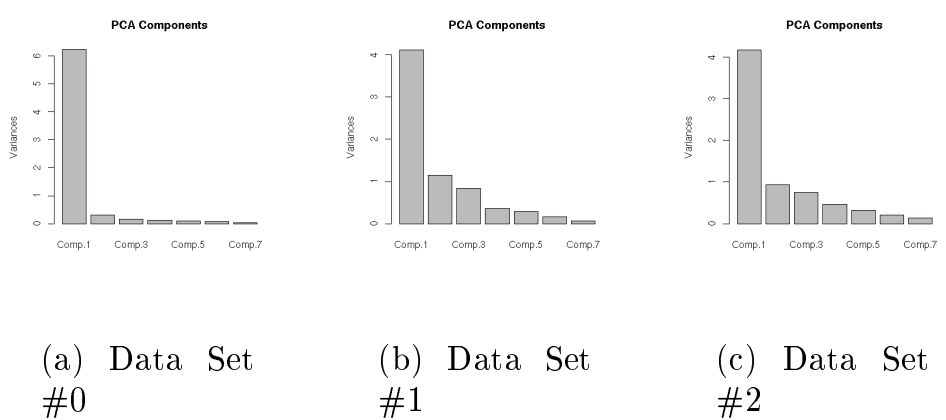

(b) Data Set \#1

(c) Data Set \#2
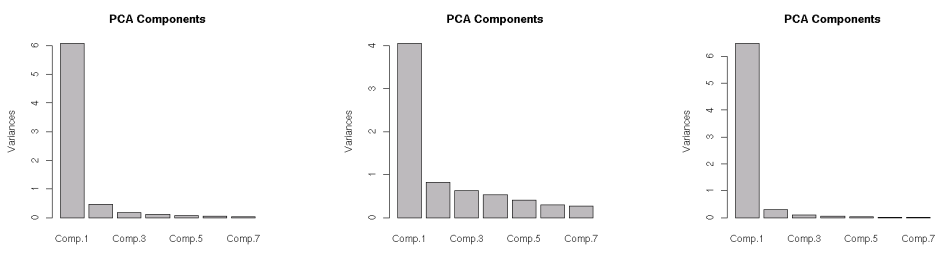

(d) Data Set \#3

(e) Data Set \#4

(f) Data Set \#5

Figure 4.3: The inbound PCA Components for the six data sets.
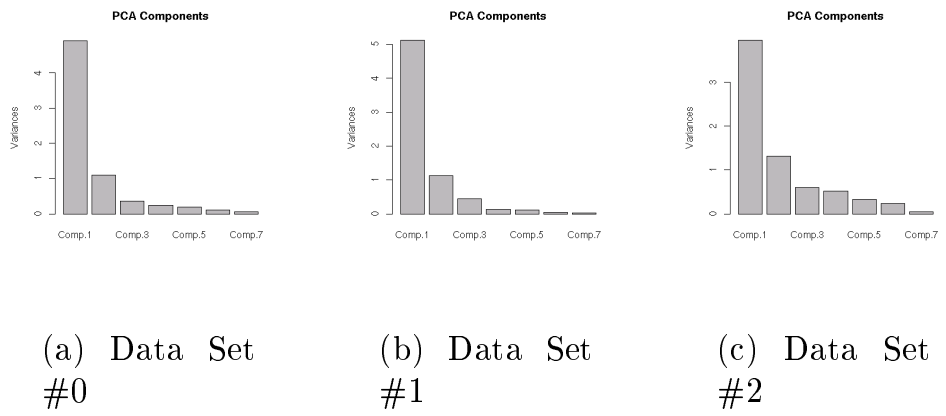

(c) Data Set \#0$$
\text { \#2 }
$$
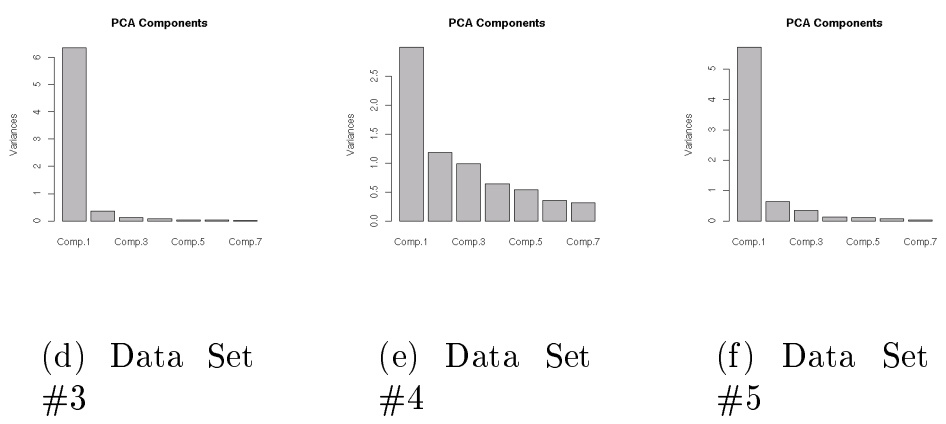
(e) Data Set \#4

(f) Data Set \#5

Figure 4.4: The outbound PCA Components for the six data sets. 


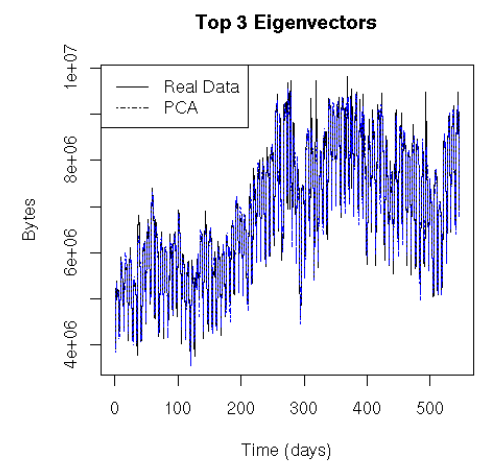

(a) Data Set \#0

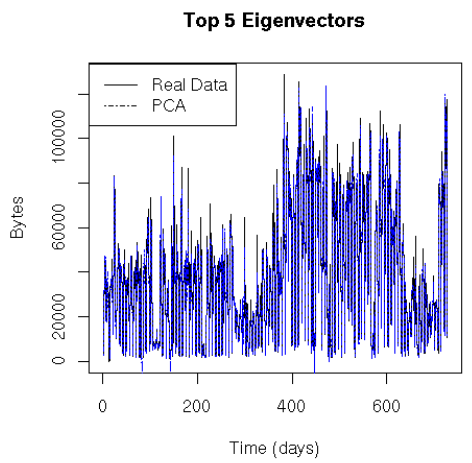

(c) Data Set \#2

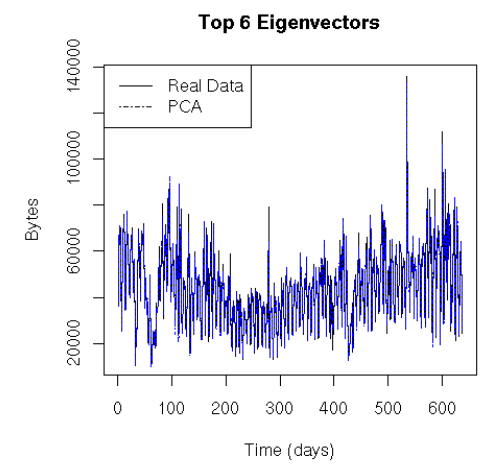

(e) Data Set \#4

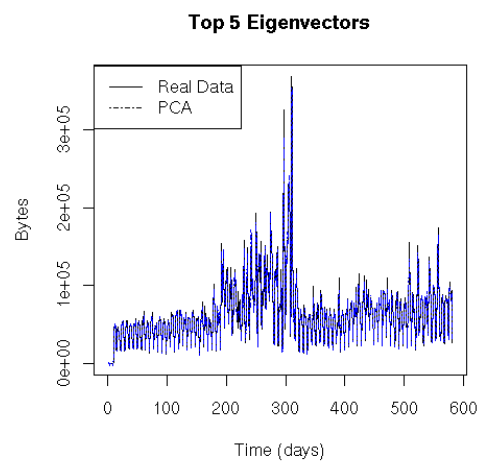

(b) Data Set \#1

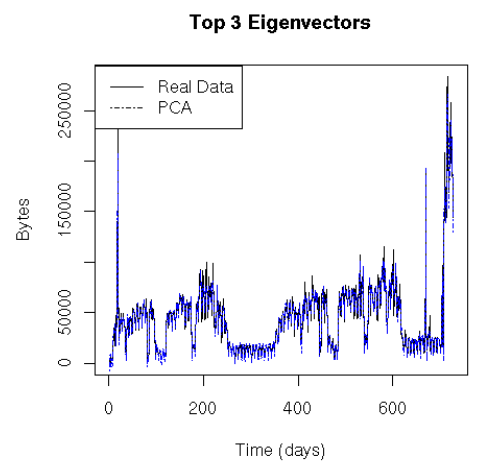

(d) Data Set \#3

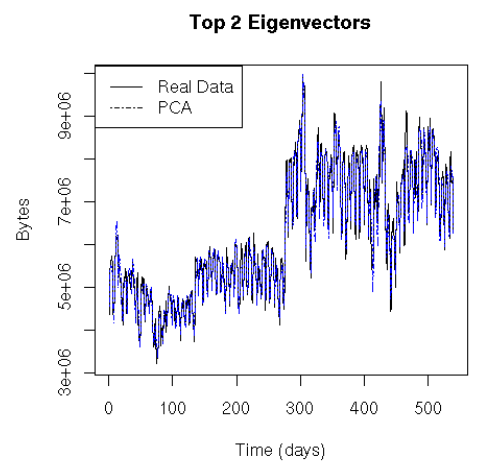

(f) Data Set \#5

Figure 4.5: The eigenvectors whose eigenvalues represent the top 95\% of the total variance were kept. The data was filtered using the remaining eigenvectors. These are the generated graphs for the inbound data on the six data sets. 


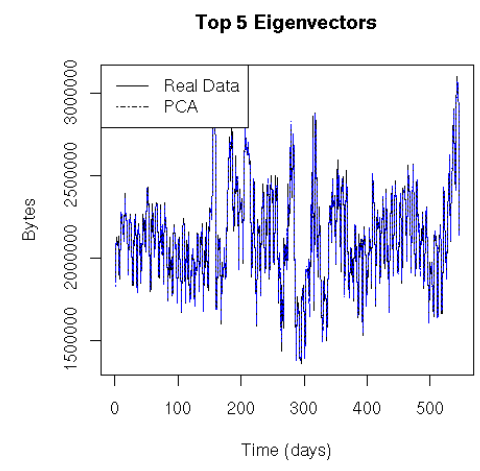

(a) Data Set \#0

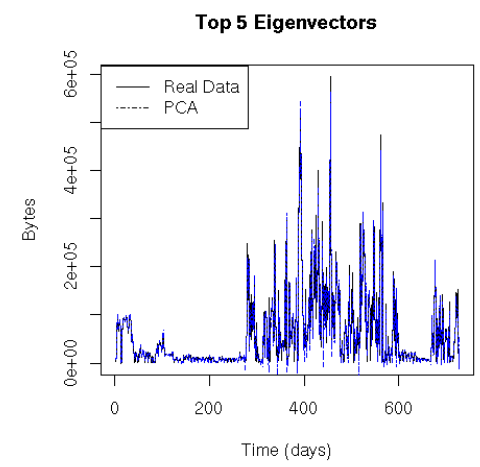

(c) Data Set \#2

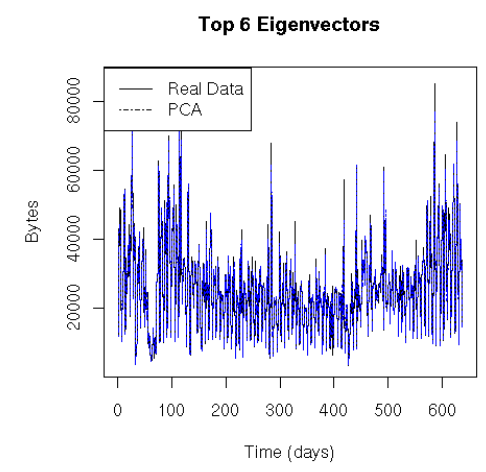

(e) Data Set \#4

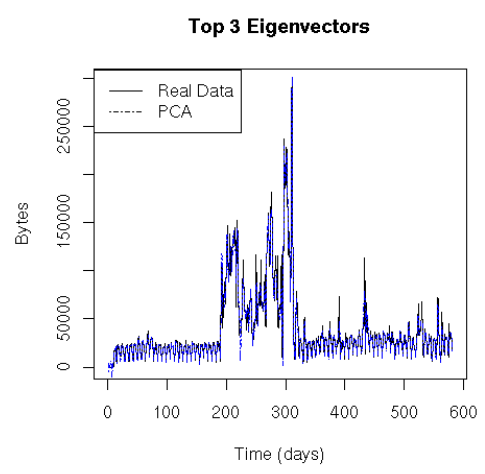

(b) Data Set \#1

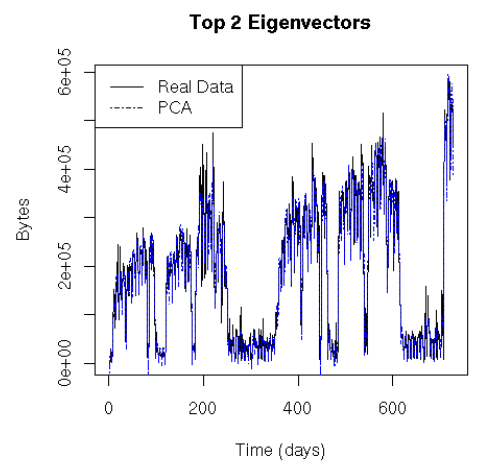

(d) Data Set \#3

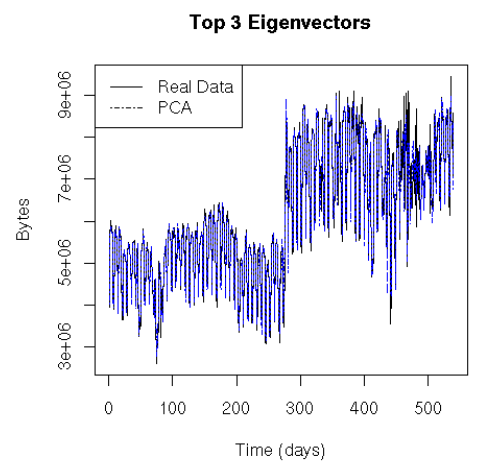

(f) Data Set \#5

Figure 4.6: The eigenvectors whose eigenvalues represent the top 95\% of the total variance were kept. The data was filtered using the remaining eigenvectors. These are the generated graphs for the outbound data on the six data sets. 
The eigenvectors whose eigenvalues represent the top $95 \%$ of the total variance were kept. The original data was filtered using PCA to remove the noise from the data set.

The ARIMA forecasting algorithm was used on all six of the PCA filtered data sets to predict the traffic utilization six months into the future. Table 4.4 shows a summary of the numeric results that were obtained. Figures 4.7 and 4.8 shows a graphical representation of the results that were obtained.

\begin{tabular}{|c|c|c|c|c|}
\hline Data Set \# & ARIMA Parameters & Root MSE (bytes) & Daily \% Error & Weekly \% Error \\
\hline \multirow{2}{*}{0} & $(11,1,11)$ & $147,353.50$ & $26.11 \%$ & $1.88 \%$ \\
& $(10,1,4)$ & $27,819.46$ & $17.31 \%$ & $2.20 \%$ \\
\hline \multirow{2}{*}{1} & $(7,1,8)$ & $4,817.29$ & $65.53 \%$ & $10.11 \%$ \\
& $(6,1,4)$ & $4,787.88$ & $80.25 \%$ & $12.60 \%$ \\
\hline \multirow{2}{*}{2} & $(9,1,8)$ & $3,867.80$ & $78.75 \%$ & $10.38 \%$ \\
& $(3,1,4)$ & $8,064.04$ & $65.49 \%$ & $20.65 \%$ \\
\hline \multirow{2}{*}{3} & $(4,1,7)$ & $1,727.11$ & $43.18 \%$ & $18.72 \%$ \\
& $(8,1,6)$ & $9,747.81$ & $47.29 \%$ & $14.46 \%$ \\
\hline \multirow{2}{*}{4} & $(6,1,7)$ & $1,364.60$ & $44.85 \%$ & $7.39 \%$ \\
& $(6,1,6)$ & $1,116.33$ & $65.29 \%$ & $8.10 \%$ \\
\hline \multirow{2}{*}{5} & $(10,1,8)$ & $186,064.10$ & $38.22 \%$ & $2.42 \%$ \\
& $(10,1,9)$ & $199,745.66$ & $43.00 \%$ & $1.38 \%$ \\
\hline
\end{tabular}

Table 4.4: The estimated ARIMA parameters for the six data sets that were smoothed using Principal Component Analysis (PCA).

\subsection{Traffic prediction using ICA and ARIMA}

In an attempt to obtain better results, the data set was smoothed using Independent Component Analysis (ICA). The training data was filtered using PCA to reduce the dimensionality of the data set. ICA was used to determine the independent components in the data set. The independent component that corresponded to the long term trend was used to filter the data set. 


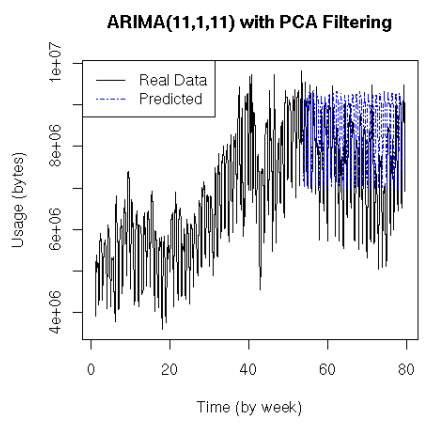

(a) Data Set \#0 Inbound

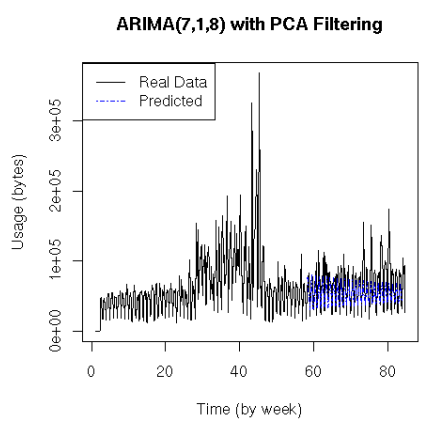

(c) Data Set \#1 Inbound

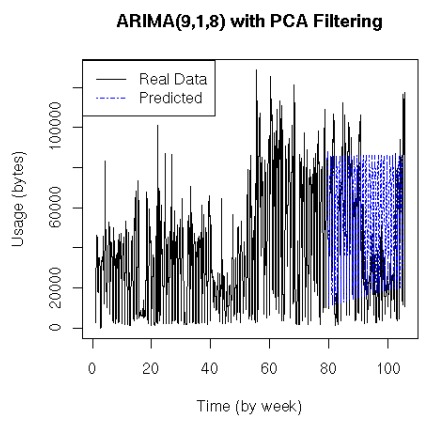

(e) Data Set \#2 Inbound

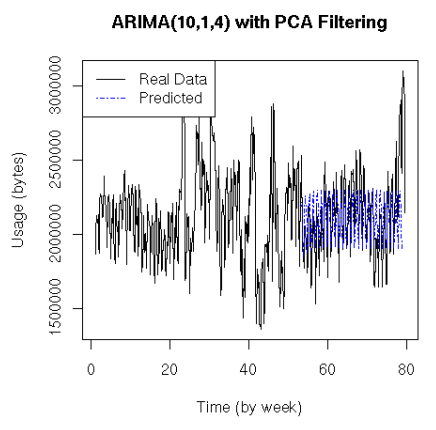

(b) Data Set \#0 Outbound

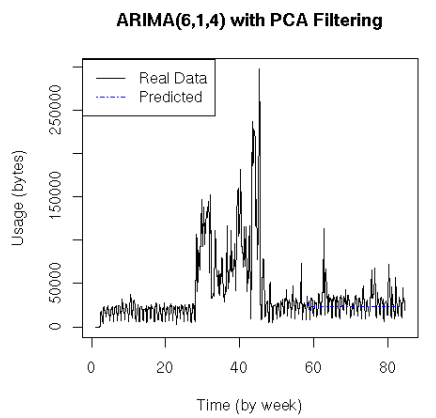

(d) Data Set \#1 Outbound

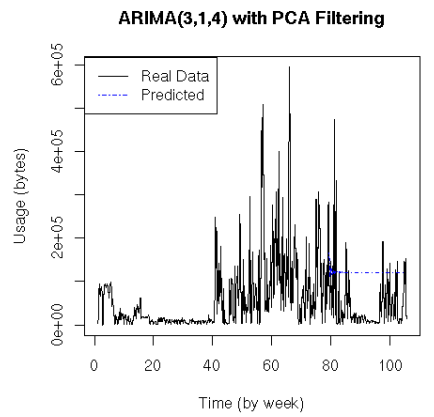

(f) Data Set \#2 Outbound

Figure 4.7: The data was smoothed using Principal Component Analysis (PCA). An ARIMA model of the smoothed data was generated for each data sets. This model was used to predict the traffic six months into the future. The results are shown for data sets \#0-2. 


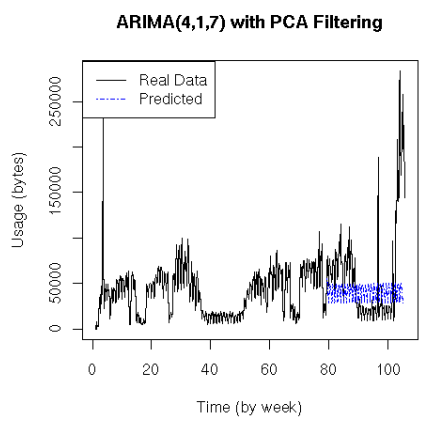

(a) Data Set \#3 Inbound

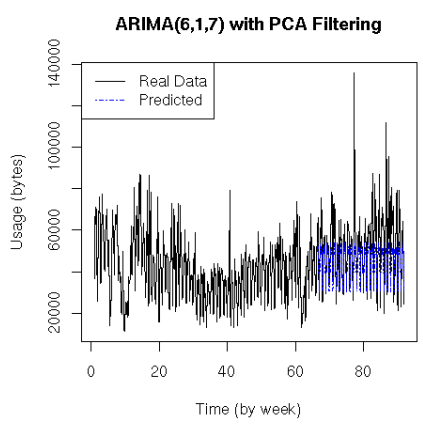

(c) Data Set \#4 Inbound

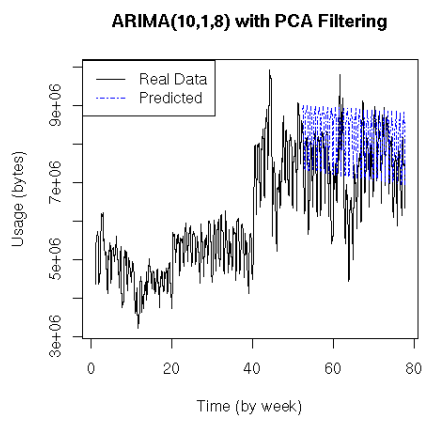

(e) Data Set \#5 Inbound

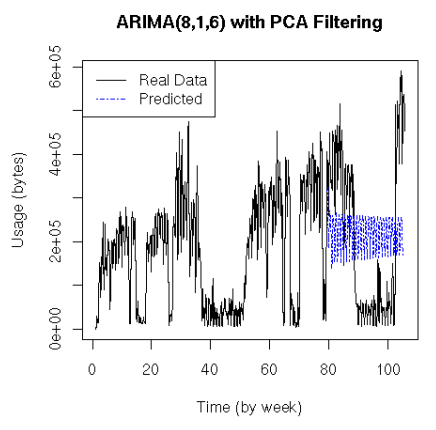

(b) Data Set \#3 Outbound

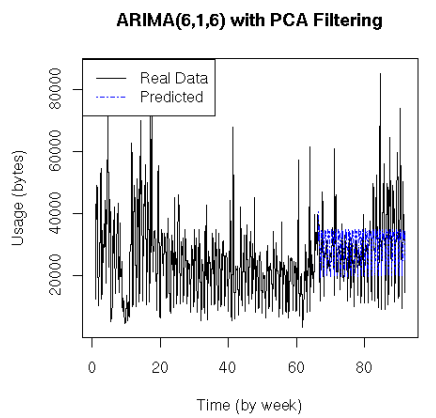

(d) Data Set \#4 Outbound

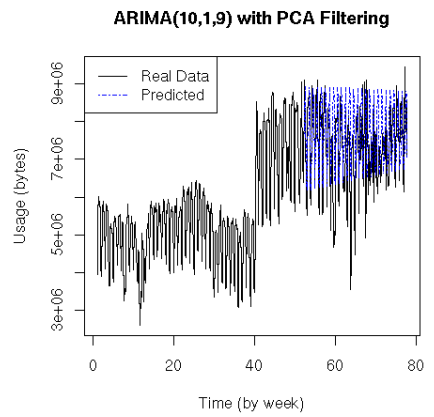

(f) Data Set \#5 Outbound

Figure 4.8: The data was smoothed using Principal Component Analysis (PCA). An ARIMA model of the smoothed data was generated for each data sets. This model was used to predict the traffic six months into the future. The results are shown for data sets \#3-5. 
The ARIMA forecasting algorithm was used on all six of the ICA filtered data sets to predict the traffic utilization six months into the future. In some cases, the ICA algorithm would not converge to a suitable value. Table 4.5 shows a summary of the numeric results that were obtained. Figures 4.9 and 4.10 shows a graphical representation of the results that were obtained.

\begin{tabular}{|c|c|c|c|c|}
\hline Data Set \# & ARIMA Parameters & Root MSE (bytes) & Daily \% Error & Weekly \% Error \\
\hline \multirow{2}{*}{0} & \multicolumn{4}{|c|}{ Did not converge. } \\
& $(0,1,9)$ & 4023.01 & $60.18 \%$ & $10.16 \%$ \\
\multirow{2}{*}{1} & $(3,1,8)$ & 3826.26 & $72.00 \%$ & $8.46 \%$ \\
\hline \multirow{2}{*}{2} & $(4,1,4)$ & 7091.34 & $74.31 \%$ & $20.21 \%$ \\
\hline \multirow{2}{*}{3} & $(7,1,4)$ & 1575.37 & $39.77 \%$ & $17.38 \%$ \\
& $(7,1,7)$ & 9646.79 & $47.14 \%$ & $13.91 \%$ \\
\hline \multirow{2}{*}{4} & $(5,1,8)$ & 1039.11 & $56.43 \%$ & $7.59 \%$ \\
\hline \multirow{2}{*}{5} & $(6,1,8)$ & 144038.92 & $29.56 \%$ & $1.69 \%$ \\
& $(10,1,9)$ & 169283.40 & $36.64 \%$ & $1.92 \%$ \\
\hline
\end{tabular}

Table 4.5: The estimated ARIMA parameters for the six data sets that were smoothed using Independent Component Analysis (ICA).

\subsection{PCA/ICA vs the STL trend component}

The smoothed data that was obtained using PCA and ICA filtering was compared with the trend component that was obtained using the STL algorithm [4]. Table 4.6 shows the correlations between all of these data sets. It appears that the seasonal component was still present in the smoothed data.

The auto-correlation function was used to verify that a seasonal component still exists in the PCA/ICA filtered data. Figure 4.11 shows a comparison between the PCA filtered data 


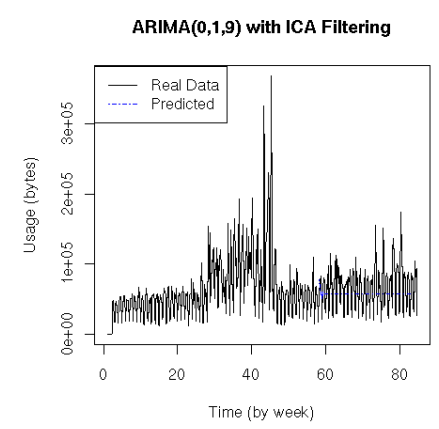

(a) Data Set \#1 Inbound

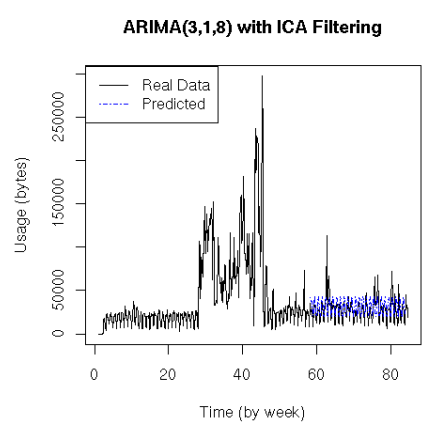

(b) Data Set \#1 Outbound

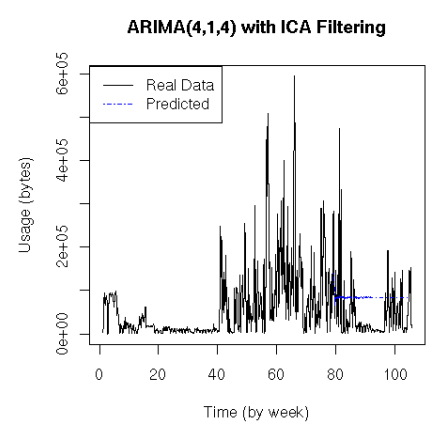

(c) Data Set \#2 Outbound

Figure 4.9: The data was smoothed using Independent Component Analysis (ICA). An ARIMA model of the smoothed data was generated for each data sets. This model was used to predict the traffic six months into the future. The results are shown for data sets \#0-2. 


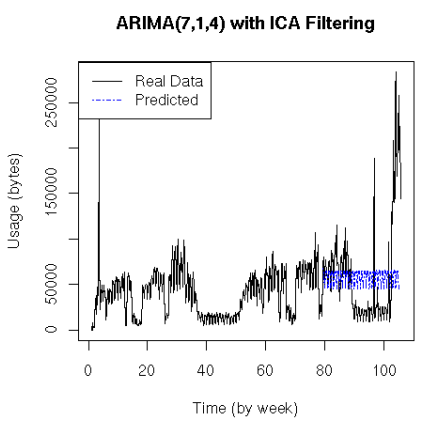

(a) Data Set \#3 Inbound

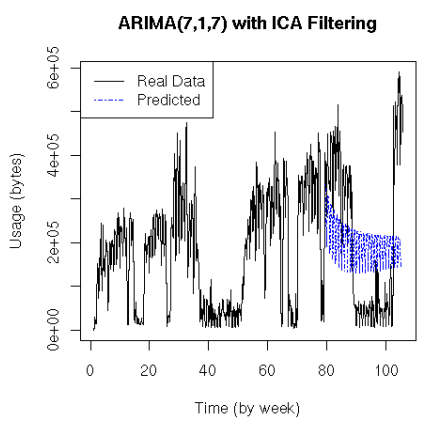

(b) Data Set \#3 Outbound

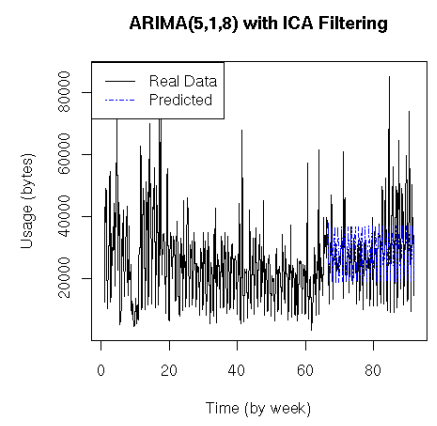

(c) Data Set \#4 Outbound

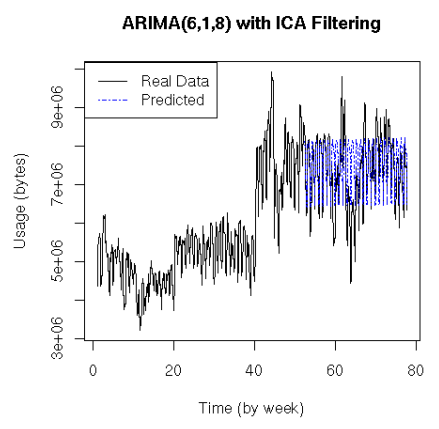

(d) Data Set \#5 Inbound

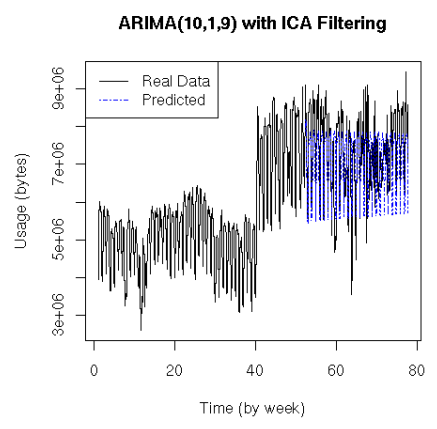

(e) Data Set \#5 Outbound

Figure 4.10: The data was smoothed using Independent Component Analysis (ICA). An ARIMA model of the smoothed data was generated for each data sets. This model was used to predict the traffic six months into the future. The results are shown for data sets \#3-5. 


\begin{tabular}{|c|c||c|c|c||c|c|}
\hline Data Set \# & in/out & $\begin{array}{c}\text { Orig. data } \\
\text { vs STL trend }\end{array}$ & $\begin{array}{c}\text { PCA data } \\
\text { vs STL trend }\end{array}$ & $\begin{array}{c}\text { ICA data } \\
\text { vs STL trend }\end{array}$ & $\begin{array}{c}\text { PCA data } \\
\text { vs orig. data }\end{array}$ & $\begin{array}{c}\text { ICA data } \\
\text { vs orig. data }\end{array}$ \\
\hline 0 & in & 0.7875 & 0.7996 & 0.7865 & 0.9617 & 0.9548 \\
0 & out & 0.7795 & 0.8140 & N/A & 0.9476 & N/A \\
\hline 1 & in & 0.6843 & 0.7211 & 0.4611 & 0.9310 & 0.8731 \\
1 & out & 0.8968 & 0.9363 & 0.8757 & 0.9484 & 0.8606 \\
\hline 2 & in & 0.6331 & 0.6642 & $\mathrm{~N} / \mathrm{A}$ & 0.9348 & $\mathrm{~N} / \mathrm{A}$ \\
2 & out & 0.8385 & 0.9328 & 0.5436 & 0.8733 & 0.7841 \\
\hline 3 & in & 0.9137 & 0.9486 & 0.9185 & 0.9289 & 0.9063 \\
3 & out & 0.9406 & 0.9420 & 0.9415 & 0.9604 & 0.9600 \\
\hline 4 & in & 0.6255 & 0.6719 & $\mathrm{~N} / \mathrm{A}$ & 0.9314 & $\mathrm{~N} / \mathrm{A}$ \\
4 & out & 0.5733 & 0.6066 & 0.4628 & 0.9414 & 0.9105 \\
\hline 5 & in & 0.9162 & 0.9203 & 0.9170 & 0.9698 & 0.9673 \\
5 & out & 0.8152 & 0.8540 & 0.8086 & 0.9265 & 0.9436 \\
\hline
\end{tabular}

Table 4.6: The correlations between the PCA and ICA smoothed data was compared against the STL trend component. It appears that the seasonal component still exists in the smoothed data.

and the STL trend. The auto-correlation function of the PCA filtered data has spikes in the graph every seven days. The auto-correlation function of the STL trend is a smooth graph.

\subsection{Anomaly detection results}

Figure 4.12 shows an example of the anomaly detection being used on one of the data sets. The original data set had an overall daily prediction error of $26 \%$. If the model is rebuilt after the first two weeks when the correlation drops below 0 , then the overall daily prediction error is $13 \%$. Rebuilding the model a second time drops the overall daily prediction error to $8 \%$. 

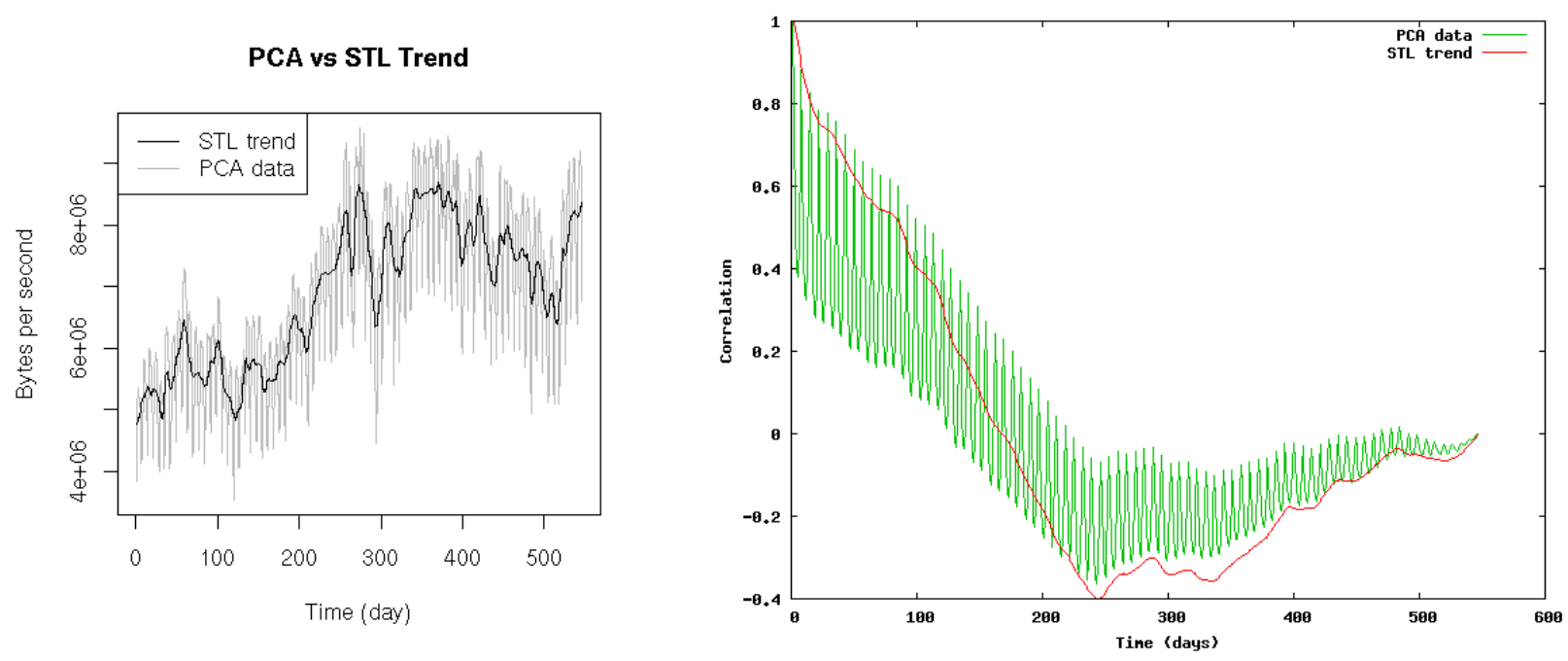

Figure 4.11: The PCA filtered data was compared against the STL trend component. The STL trend component is much smoother in appearance. The auto-correlation function of both data sets was computed. This shows that a seasonal component still exists in the PCA filtered data set. Similar results were obtained for the ICA filtered data set.

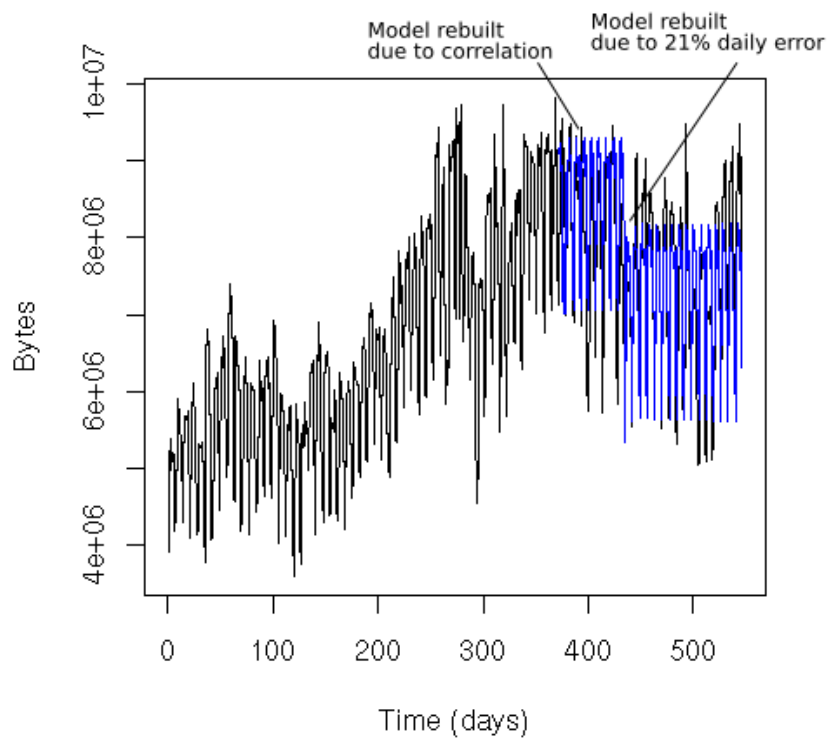

Figure 4.12: The original data set had an overall daily prediction error of $26 \%$. If the model is rebuilt after the first two weeks when the correlation drops below 0 , then the overall daily prediction error is $13 \%$. Rebuilding the model a second time drops the overall daily prediction error to $8 \%$. 


\subsection{Summary}

Figure 4.7 shows a summary of the results that were obtained using the three prediction schemes. The data sets that had large fluctuations had a higher percentage error.

\begin{tabular}{|c|c|c|c|}
\hline Data Set \# & \% Error & \% PCA Error & \% ICA Error \\
\hline \multirow{2}{*}{0} & $2.13 \%$ & $1.88 \%$ & $\mathrm{NA}$ \\
& $2.56 \%$ & $2.20 \%$ & $\mathrm{NA}$ \\
\hline \multirow{2}{*}{1} & $8.32 \%$ & $10.11 \%$ & $10.16 \%$ \\
& $13.90 \%$ & $12.60 \%$ & $8.46 \%$ \\
\hline \multirow{2}{*}{2} & $10.04 \%$ & $10.38 \%$ & $\mathrm{NA}$ \\
& $20.33 \%$ & $20.65 \%$ & $20.21 \%$ \\
\hline \multirow{2}{*}{3} & $17.01 \%$ & $18.72 \%$ & $17.38 \%$ \\
& $19.52 \%$ & $14.46 \%$ & $13.91 \%$ \\
\hline \multirow{2}{*}{4} & $7.15 \%$ & $7.39 \%$ & $\mathrm{NA}$ \\
& $7.84 \%$ & $8.10 \%$ & $7.59 \%$ \\
\hline \multirow{2}{*}{5} & $2.56 \%$ & $2.42 \%$ & $1.69 \%$ \\
& $1.42 \%$ & $1.38 \%$ & $1.92 \%$ \\
\hline
\end{tabular}

Table 4.7: A summary of the weekly percentage error for the three ARIMA prediction schemes.

\subsection{Future Work}

- The PCA/ICA filtered data contained the overall long-term trend along with the seasonal component. Another layer of filtering can be applied in order to remove the seasonal component.

- This thesis only used the ARIMA family of the functions. It would be worthwhile to try modeling the data with a fractal based model.

- Recall that a time series can be represented as the sum of three components: trend, seasonal and noise. It may be possible to model the noise and use this to derive the 
trend and seasonal components.

- The prediction scheme utilized in this thesis does not take into account the data from the marketing and managerial personnel. An advertising campaign in a particular region is likely to cause an increase in business in that area. A prediction scheme should be be able to accept some of this external data. 


\section{BIBLIOGRAPHY}

[1] Brockwell, Peter J. and Davis, Richard A. (2002). Introduction to Time Series and Forecasting, 2nd. ed., Springer-Verlang.

[2] J Brutlag. Aberrant Behavior Detection in Time Series for Network Monitoring. Proceedings of the USENIX Fourteenth System Administration - usenix.org. http://www.usenix.org/events/lisa2000/full_papers/brutlag/brutlag_html/

[3] M.F. Barnsley, Fractal modelling of real world images, in: The Science of Fractal Images, H.-O. Peitgen and D. Saupe

[4] R. Cleveland, W. Cleveland, J. McRae and I. Terpenning, "STL: A Seasonal-Trend Decomposition Procedure Based on Loess," in the Journal of Official Statistics, Vol. 6, No. 1, 1990, pp.3-73.

[5] M. Grossglauser and J. Bolot. On the Relevance of Long-Range Dependence in Network Traffic, IEEE/ACM Transactions on Networking, Vol. 7, No. 5, Oct. 1999.

[6] R. Duda, P. Hart and D. Stork (October 2000). Pattern Classification, 2nd. ed., Wiley Interscience. 
[7] Eke, A., Hermn, P., Kocsis, L., and Kozak, L. R. (2002). Fractal characterization of complexity in temporal physiological signals. Physiological Measurement, 23, R1R38.

[8] W. E. Leland, M. S. Taqqu, W. Willinger and D. V. Wilson. On the self-similar nature of Ethernet traffic (extended version). IEEE/ACM Transactions on Networking, 2(1):1-15, 1994.

[9] Mandelbrot B (1982). The Fractal Geometry of Nature, New York: W. H. Freeman.

[10] A. Media, N. Talf, K. Salamatian, S. Bhattacharyya and C. Diot. Traffic Matrix Estimation: Existing Techniques and New Directions. Proceedings of the 2003 conference on Applications, technologies, architectures, and protocols for computer communications, pp. $301-312,2003$.

[11] C. Molina-Jimenez, S. Shrivastava, J. Crowcroft and P. Gevros. On the Monitoring of Contractual Service Level Agreements. Proceedings of the First International Workshop on Electronic Contracting (WEC '04).

[12] K. Papagiannaki, N. Taft and A. Lakhina. A Distributed Approach to Measure IP Traffic Matrices. Proceedings of the 4th ACM SIGCOMM conference on Internet measurement, pp. $161-174,2004$.

[13] K. Papagiannaki, N. Taft, Z. Zhang, C. Diot, Long-Term Forecasting of Internet Backbone Traffic: Observations and Initial Models, Communications Magazine, IEEE INFOCOM, 2003. 
[14] V. Paxson and S. Floyd, "Wide area traffic: The failure of Poisson modeling," IEEE/ACM Trans. Networking, vol. 3, pp. 226244, June 1995.

[15] Peitgen H-O, Jurgens H and Saupe D. Chaos and Fractals, New Frontiers of Science (New York: Springer), 1992.

[16] Resnick, S. and Starica, Catalin, Tail Index estimation for dependent data, Available as TR1174.ps.Z at http://www.orie.cornell.edu/trlist/trlist.html, Preprint (1997). http://citeseer.ist.psu.edu/resnick97tail.html

[17] O. Rose. Estimation of the Hurst Parameter of Long-Range Dependent Time Series. February 1996. http://citeseer.ist.psu.edu/rose96estimation.html

[18] R. Ritke, X. Hong, M. Gerla, Contradictory relationship between Hurst parameter and queueing performance (extended version), Telecomm. Systems 16 (1,2) (2001) 159-175.

[19] A. Sang and S. Li. A Predictability Analysis of Network Traffic, Computer Networks, 2002.

[20] W. Stallings, High Speed Networks; TCP/IP ATM Design Principles, Prentice Hall, 1998, pp. 181-207.

[21] Z. Sahinoglu, S. Tekinay, On Multimedia Networks: Self Similar Traffic and Network Performance, Communications Magazine, IEEE, Jan. 1999, pp. 48-52.

[22] A. Veres, Z. Kenesi, S. Molnar and G. Vattay. On the Propagation of Long-Range Dependence in the Internet, SIGCOMM, 2000. 
[23] S. Walker, A Primer on Wavelets and their Scientific Applocations. Chapman \& Hall, 1999.

[24] W. Willinger, M. Taqqu, R. Sherman, and D. Wilson, Self-similarity through high variability: Statistical analysis of Ethernet LAN traffic at the source level, IEEE/ACM Trans. Networking, vol. 5, pp. 7186, Feburary 1997.

[25] G. Nason and B. Silverman, "The Stationary Wavelet Transform and some Statistical Applications," in Lecture Notes in Statistics: Wavelets and Statistics, 1995, pp. 281300. [Online]. Available: citeseer.nj.nec.com/nason95stationary.html

[26] M. Shensa, "The Discrete Wavelet Transform: Wedding the A Trous and Mallat Algorithms," in IEEE Transactions on Signal Processing, vol. 40, no. 10, 1992, pp. 24642482.

[27] ANOVA http://www.sixsigmafirst.com/anova.htm

[28] Autoregressive moving average model http://en.wikipedia.org/wiki/Autoregressive_moving_average_model

[29] Autocorrelation http://en.wikipedia.org/wiki/Autocorrelation

[30] SNMP Counters: Frequently Asked Questions http://www.cisco.com/warp/public/477/SNMP/faq-snmpcounter.shtml

[31] ICA cocktail party demo http://www.cis.hut.fi/projects/ica/cocktail/cocktail_en.cgi

[32] List of Device Bandwidths http://en.wikipedia.org/wiki/List_of_device_bandwidths 
[33] Lag operator http://en.wikipedia.org/wiki/Lag_operator

[34] Long-tail traffic http://en.wikipedia.org/wiki/Long-tail_traffic

[35] Long-range dependency http://en.wikipedia.org/wiki/Long-range_dependency

[36] LOESS http://www.itl.nist.gov/div898/handbook/pmd/section1/pmd144.htm

[37] RFC768: User Datagrap Protocol http://www.ietf.org/rfc/rfc768

[38] RFC2309: Recommendations on Queue Management and Congestion Avoidance in the Internet http://www.ietf.org/rfc/rfc2309

[39] rrdcreate man page http://man-wiki.net/index.php/1:rrdcreate

[40] RRDtool http://ee-staff.ethz.ch/ oetiker/webtools/rrdtool/

[41] RRD World http://people.ee.ethz.ch/ oetiker/webtools/rrdtool/rrdworld/index.en.html

[42] Seasonality http://www.itl.nist.gov/div898/handbook/pmc/section4/pmc443.htm

[43] Simple Network Management Protocol (SNMP) http://www.snmplink.org/

[44] List of Tier-1 ISPs http://en.wikipedia.org/wiki/List_of_tier_1_internet_service_providers 